\title{
The Interaction of High-Speed Turbulence with Flames: Turbulent Flame Speed
}

\author{
A.Y. Poludnenko*, E.S. Oran \\ Laboratory for Computational Physics and Fluid Dynamics, Naval Research Laboratory, Washington, DC 20375, USA
}

\begin{abstract}
Direct numerical simulations of the interaction of a premixed flame with driven, subsonic, homogeneous, isotropic, Kolmogorov-type turbulence in an unconfined system are used to study the mechanisms determining the turbulent flame speed, $S_{T}$, in the thin reaction zone regime. High intensity turbulence is considered with the r.m.s. velocity 35 times the laminar flame speed, $S_{L}$, resulting in the Damköhler number $D a=0.05$. The simulations were performed with Athena-RFX, a massively parallel, fully compressible, high-order, dimensionally unsplit, reactive-flow code. A simplified reaction-diffusion model, based on the one-step Arrhenius kinetics, represents a stoichiometric $\mathrm{H}_{2}$-air mixture under the assumption of the Lewis number $L e=1$. Global properties and the internal structure of the flame were analyzed in an earlier paper, which showed that this system represents turbulent combustion in the thin reaction zone regime. This paper demonstrates that: (1) The flame brush has a complex internal structure, in which the isosurfaces of higher fuel mass fractions are folded on progressively smaller scales. (2) Global properties of the turbulent flame are best represented by the structure of the region of peak reaction rate, which defines the flame surface. (3) In the thin reaction zone regime, $S_{T}$ is predominantly determined by the increase of the flame surface area, $A_{T}$, caused by turbulence. (4) The observed increase of $S_{T}$ relative to $S_{L}$ exceeds the corresponding increase of $A_{T}$ relative to the surface area of the planar laminar flame, on average, by $\approx 14 \%$, varying from only a few percent to as high as $\approx 30 \%$. (5) This exaggerated response is the result of tight flame packing by turbulence, which causes frequent flame collisions and formation of regions of high flame curvature $\gtrsim 1 / \delta_{L}$, or "cusps," where $\delta_{L}$ is the thermal width of the laminar flame. (6) The local flame speed in the cusps substantially exceeds its laminar value, which results in a disproportionately large contribution of cusps to $S_{T}$ compared with the flame surface area in them. (7) A criterion is established for transition to the regime significantly influenced by cusp formation. In particular, at Karlovitz numbers $K a \gtrsim 20$, flame collisions provide an important mechanism controlling $S_{T}$, in addition to the increase of $A_{T}$ by large-scale motions and the potential enhancement of diffusive transport by small-scale turbulence.
\end{abstract}

Keywords: Turbulent premixed combustion, Turbulence, Flamelet, Turbulent flame speed, Hydrogen-air combustion

\section{Introduction}

One of the fundamental questions of turbulent combustion research concerns our ability to understand and predict the rate of energy release, or equivalently the burning speed, of a turbulent flame. This can be achieved if two key aspects of the combustion process can be determined: (1) its local properties, namely the local speed of flame propagation, and (2) its global characteristics, i.e., the overall structure of the turbulent flame that connects the local burning velocity with the turbulent flame speed. In general, such local and global characteristics are not universal for the combustion process. They can vary substantially both in space and time due to the unsteadiness, inhomogeneity, and anisotropy of the turbulent flow associated with the system geometry, presence of walls and boundaries, change in the flow conditions, etc.

Our present understanding of turbulent combustion is largely based on the concept originally proposed almost 70 years ago by Damköhler [1] (also see reviews by [2, 3, 4, 5]). This concept is formulated for a turbulent flame

\footnotetext{
${ }^{*}$ Corresponding author. Fax: +12027674798.

Email address: apol@lcp.nrl.navy.mil (A.Y. Poludnenko)
} 
that is, on average, a planar, quasi-one-dimensional structure propagating in a steady, homogeneous, and isotropic background turbulent flow. According to Damköhler's suggestion, two qualitatively distinct regimes of turbulenceflame interaction can be identified, and they are typically referred to as "large-scale" and "small-scale" turbulence [1, 5].

In the first regime, the overall structure of the turbulent flame is determined by turbulent motions on large scales greater than the flame width, $\delta_{L}$. These motions stretch and fold the flame, thereby increasing its surface area, $A_{T}$. At the same time, they do not affect the local burning velocity, $S_{n}$, with which the flame propagates normal to its surface at each point and which remains equal to the speed, $S_{L}$, of the unperturbed laminar flame. Consequently, the speed, $S_{T}$, of the turbulent flame grows proportionally to the increase of the flame surface area, i.e., $S_{T}=S_{L}\left(A_{T} / A_{L}\right)$, where $A_{L}$ is the surface area of the planar laminar flame. The limitations of this assumption were known practically at the time when this model was proposed [6]. In particular, when the Lewis number $L e \neq 1, S_{n}$ can vary with the curvature of the flame. As a result, the stretching and folding action of scales $\lambda>\delta_{L}$ ends up affecting $S_{n}$. The relation between $A_{T}$ and $S_{T}$, however, can be generalized by incorporating the effects of flame strain and curvature in terms of the stretch factor $I$ ([3], also see [7] for the review of the theory of flame stretch),

$$
\frac{S_{T}}{S_{L}}=I \frac{A_{T}}{A_{L}}
$$

In the second regime, large-scale wrinkling of the flame by turbulence is absent. Instead, small-scale motions, which are energetic enough, penetrate and disrupt the internal flame structure. Thus molecular diffusivity and thermal conduction become enhanced, or completely dominated, by the turbulent transport associated with such small-scale turbulence. As a result, in this regime, the turbulent flame speed is suggested to be [1]

$$
\frac{S_{T}}{S_{L}} \sim\left(\frac{D_{T}}{D}\right)^{1 / 2},
$$

where $D$ and $D_{T}$ are, respectively, the molecular and the effective turbulent diffusivities.

These two regimes, in effect, represent two limits of low and high turbulent intensity. In the first case, large-scale turbulence must be slow enough so that the Kolmogorov scale $\eta>\delta_{L}$, thus causing the flow to remain laminar on scales $\lambda \lesssim \delta_{L}$. This combustion regime corresponds to the "wrinkled" and "corrugated flamelets." In the opposite limit, the small-scale turbulence regime is realized when turbulent motions on all scales in the system are sufficiently fast to form an effectively one-dimensional (1D) structure analogous to the planar laminar flame, but which has a much larger width and speed. This second regime is often associated with either a "thin reaction zone" flame [5] or a "broken reaction zone" ("distributed") flame [4, 8, 9, 10]. As a consequence of considering these two limits, description of the turbulent flame becomes significantly simplified. The large-scale regime assumes very simple local flame properties, i.e., those of the laminar flame, but a complex global structure of a highly convolved flame surface. The small-scale regime, on the other hand, trades the simplicity of the global structure of a planar flame for the complexity of its internal structure, which is pronouncedly distinct from that of the laminar flame. Therefore, each of these regimes has only one dynamically important quantity that completely determines $S_{T}$, namely $A_{T}$ for the large-scale turbulence and $D_{T}$ for the small-scale turbulence.

This picture then naturally raises the following question. How does the transition occur between these two limiting regimes of combustion? At some intermediate turbulent intensities, the flow will cease to be laminar on scales $\lambda \lesssim \delta_{L}$. As a result, turbulence will begin to penetrate the flame, disrupting it and causing its internal structure to differ from that of the laminar flame. At the same time, the flame can remain sufficiently thin to form, under the action of highintensity large-scale motions, a highly convolved and tightly packed configuration. The picture outlined above would then imply a gradual shift with increasing turbulent intensity from the large-scale turbulence being the dominant process controlling $S_{T}$ to the small-scale one. Over a substantial range of turbulent intensities, however, it must be their combined action that determines the magnitude of $S_{T}$.

Of particular interest, in this respect, is the "thin reaction zone" regime, in which only the preheat zone is disrupted by turbulence. The presence of turbulent motions on scales $\lambda \lesssim \delta_{L}$ suggests that the molecular and thermal transport between the reaction and preheat zones are affected by turbulence. This should cause $S_{n}$ to differ from $S_{L}$, which is characteristic of the small-scale turbulence regime. On the other hand, if the structure of the reaction zone is the 
same as in the laminar case, as occurs at the large-scale boundary of this regime, then $S_{n}$ must equal $S_{L}$. Indeed, $S_{n}=\left(1 / \rho_{0}\right) \int \rho \dot{Y} d \zeta$, where $\rho_{0}$ is the fuel density, $\dot{Y}$ is the reaction rate, and $\zeta$ is the coordinate normal to the flame. Since $\dot{Y} \neq 0$ only in the reaction zone where it does not differ from the laminar flame, it follows that $S_{n}=S_{L}$. What does then determine $S_{T}$ in the thin reaction zone regime? Is it folding and stretching of the flame by turbulence, implying the validity of eq. (1), or the microscopic turbulent transport represented by eq. (2), or both? How does the balance between the two processes change with turbulent intensity? Finally, is the effect of high-speed turbulence on the flame limited to the two processes discussed above or do other mechanisms also play a role?

Before these questions can be addressed, several conceptual difficulties must first be resolved. Most importantly, the definition of the flame surface and its area must be revisited. This question, which has a seemingly simple answer at low turbulent intensities, becomes quite nontrivial when the flow is no longer laminar on scales $\lambda \lesssim \delta_{L}$. In this case, different regions of the flame are affected differently by turbulence and, thus, the isosurfaces of temperature or fuel mass fraction are not necessarily parallel. Furthermore, without an accurate definition of the flame surface, it is not clear what the local burning speed of the flame is, since this quantity typically describes flame propagation normal to its surface and determining its local value requires integration of the distribution of $\dot{Y}$ normal to the flame surface, as was discussed above.

In recent years, the advent of direct numerical simulations (DNS) has provided a tool permitting the mechanisms that determine $S_{T}$ to be directly investigated. DNS have been used by a number of groups to study the dynamics and properties of turbulent flames [9]-[11] (also see a review by [22]). With the exception of the work by Aspden et al. [9, 10] that analyzed the fully distributed thermonuclear flame, these studies generally assume relatively low turbulent intensities. Several authors also did explicitly consider the relation between $S_{T}$ and $A_{T}$. Bell et al. [20] analyzed the three-dimensional (3D) DNS of a statistically planar turbulent methane flame and determined that $I$ deviates from unity by $\lesssim 10 \%$. A similar estimate was obtained by Khokhlov [23] in 3D simulations of Rayleigh-Taylor driven thermonuclear flames in degenerate matter. Both of these studies, however, considered reactive mixtures in which $L e \neq 1$ and, therefore, $I$ would not be expected to be exactly equal to one. In this context, the result of [23] is particularly interesting since degenerate matter is characterized by Le $\gg 1$ and typically in excess of $10^{3}$. Furthermore, both Bell et al. [20] and Khokhlov [23] considered turbulent intensities which were too low to allow small-scale motions to penetrate efficiently the interior of the flame. For instance, in [20], the characteristic turbulent velocity was $\approx 4.3 S_{L}$, and in [23], it reached values $\lesssim 12 S_{L}$. Consequently, no effects of the microscopic turbulent transport would be expected in these cases.

In this respect, the work of Hawkes \& Chen [21] is of particular interest as it considered the validity of eq. (1) in the thin reaction zone regime. They analyzed simulations of lean, statistically flat turbulent methane flames at turbulent intensities $\lesssim 28.5 S_{L}$ and found values of $I$ within a few percent of unity, with the exception of the methane-hydrogen flame for which $I=1.18$ was determined. Interpretation of the implications of these results for the thin reaction zone regime, however, is complicated by the two-dimensional (2D) nature of their simulations. Moreover, the turbulent integral scale was $<\delta_{L}$, which substantially suppressed flame wrinkling and prevented the development of a highly convolved flame which would typically be expected to form in a realistic setting.

The studies performed to-date, thus, generally show that, at low turbulent intensities, the increase in $S_{T}$ is indeed almost completely determined by the growth of $A_{T}$. At the same time, they leave open the questions discussed above regarding the mechanisms controlling $S_{T}$ in the thin reaction zone regime, let alone at higher turbulent intensities.

The objective of this paper is to begin addressing these questions systematically by first considering the interaction of a statistically planar, turbulent flame with the steady, homogeneous, isotropic turbulence. We assume sufficiently high turbulent intensity to represent the regime that is borderline between thin and broken reaction zones, according to the traditional combustion regime diagrams [5, 24, 25] (cf. Fig. 2 in [11]). In other words, we consider the highestintensity turbulence, which has been hypothesized to allow for the existence of a flame with the internal structure of the reaction zone that is essentially not affected by turbulent transport. The turbulent r.m.s. velocity of the flow in cold fuel is $U_{r m s} \approx 35 S_{L}$, leading to the Damköhler number $D a=0.05$ and Gibson scale $L_{G} \approx 3 \times 10^{-4} \delta_{L}$. Here we focus on the $L e=1$ situation to exclude thermodiffusive effects as a potential source of variations of the local flame speed. Developing rigorous understanding of this simpler case will then serve as a starting point for the study of more realistic situations represented by more complex chemistry.

This paper continues the analysis of the simulations first presented in [11]. The primary focus of [11] was a detailed study of flame properties and evolution in the presence of such high-speed turbulence. In particular, a method 
was presented which allowed the direct determination of the internal structure of the flame based on its actual 3D configuration inside the flame brush. The analysis showed that the preheat zone is broadened by turbulence while the reaction-zone structure remains virtually identical to that of the planar laminar flame. Therefore, this study demonstrated that, even under the action of such intense turbulence, the system can be classified as being in the thin reaction zone regime.

Our starting point in this work is the question of the definition of the flame surface area in the high-speed turbulent flow. Answering this will then allow us to determine whether a relation between $S_{T}$ and $A_{T}$, similar to eq. (1), can indeed be established and, thus, which processes control $S_{T}$ in the thin reaction zone regime.

\section{Numerical method and simulations performed}

\subsection{Physical and numerical models}

Here we summarize the physical model, the numerical method used, and key aspects of the simulation setup. A more detailed discussion can be found in [11].

We solve the system of unsteady, compressible, reactive flow equations,

$$
\begin{aligned}
\frac{\partial \rho}{\partial t}+\nabla \cdot(\rho \mathbf{u}) & =0, \\
\frac{\partial \rho \mathbf{u}}{\partial t}+\nabla \cdot(\rho \mathbf{u} \otimes \mathbf{u})+\nabla P & =0, \\
\frac{\partial E}{\partial t}+\nabla \cdot((E+P) \mathbf{u})-\nabla \cdot(\mathcal{K} \nabla T) & =-\rho q \dot{Y}, \\
\frac{\partial \rho Y}{\partial t}+\nabla \cdot(\rho Y \mathbf{u})-\nabla \cdot(\rho D \nabla Y) & =\rho \dot{Y} .
\end{aligned}
$$

Here $\rho$ is the mass density, $\mathbf{u}$ is the velocity, $E$ is the energy density, $P$ is the pressure, $Y$ is the mass fraction of the reactants, $q$ is the chemical energy release, and $\dot{Y}$ is the reaction source term. The coefficients of thermal conduction, $\mathcal{K}$, and molecular diffusion, $D$, are

$$
D=D_{0} \frac{T^{n}}{\rho}, \quad \frac{\mathcal{K}}{\rho C_{p}}=\kappa_{0} \frac{T^{n}}{\rho},
$$

where $D_{0}, \kappa_{0}$, and $n$ are constants, and $C_{p}=\gamma R / M(\gamma-1)$ is the specific heat at constant pressure. The equation of state is that of an ideal gas. Chemical reactions are modeled using the first-order Arrhenius kinetics

$$
\frac{d Y}{d t} \equiv \dot{Y}=-\rho Y B \exp \left(-\frac{Q}{R T}\right)
$$

where $B$ is the pre-exponential factor, $Q$ is the activation energy, and $R$ is the universal gas constant.

Table 2 summarizes the parameters of the physical model used as well as the resulting properties of the planar laminar flame [11]. These parameters are based on a simplified reaction-diffusion model designed to represent the stoichiometric $\mathrm{H}_{2}$-air mixture [26]. We refer to [11, 26] for the detailed discussion of the limitations of applicability of this model for the description of the actual $\mathrm{H}_{2}$-air mixture.

Eqs. (3)-(8) are solved using the code Athena-RFX [11] - the reactive flow extension of the magnetohydrodynamic code Athena [27,28]. Athena-RFX is a fixed-grid, massively parallel, finite-volume, fully conservative code. It implements a variant [28] of the fully unsplit corner-transport upwind (CTU) algorithm [29] and its 3D extension presented in [30], in conjunction with the PPM spatial reconstruction [31] and the approximate nonlinear HLLC Riemann solver. Overall, the code achieves $3^{\text {rd }}$-order accuracy in space and $2^{\text {nd }}$-order accuracy in time. A detailed description and an extensive suite of tests of the hydrodynamic integration algorithm can be found in [27, 28]. Implementation of the reactive-diffusive extensions in Athena-RFX, along with the results of tests including convergence studies, is discussed in detail in [11, 32].

The simulations presented here model flame interaction with steady, homogeneous, isotropic turbulence, described by the classical Kolmogorov theory [33]. Turbulence driving is implemented using a spectral method [11, 32] similar to the one used in [34, 35]. In this method, velocity perturbations $\delta \hat{\mathbf{u}}(\mathbf{k})$ are initialized in Fourier space, and each 
component $\delta \hat{u}_{i}^{\prime}(\mathbf{k})$ is an independent realization of a Gaussian random field superimposed with the desired energy injection spectrum. Subsequently, nonsolenoidal components of $\delta \hat{\mathbf{u}}(\mathbf{k})$ are removed to ensure that $\nabla \cdot \delta \mathbf{u}(\mathbf{x})=0$. An inverse Fourier transform of $\delta \hat{\mathbf{u}}(\mathbf{k})$ gives $\delta \mathbf{u}(\mathbf{x})$, the velocity perturbation field in the physical space. The $\delta \mathbf{u}(\mathbf{x})$ is normalized to provide a constant rate $\varepsilon$ of kinetic-energy injection, and the total momentum in the perturbation field is subtracted from $\delta \mathbf{u}(\mathbf{x})$ to ensure that no net momentum is added to the domain, i.e., $\int \rho \delta \mathbf{u}=0$. The resulting velocity perturbations are added to the flow field $\mathbf{u}(\mathbf{x})$ at every time step, and the overall perturbation pattern is regenerated at every time interval $\Delta t_{v p} \sim 10 \Delta x / c_{s}$, where $c_{s}$ is the maximum sound speed in the domain and $\Delta x$ is the computational cell size.

Energy is injected only at the scale of the domain width, $L$, to obtain the Kolmogorov-type spectrum. The resulting turbulence is statistically steady, isotropic, and homogeneous with the inertial range of the energy cascade extending all the way to the energy injection scale (Fig. 1). Moreover, since the velocity perturbation field is divergence-free, no compressions or rarefactions are artificially induced as a result of driving.

Eqs. (3)-(6) do not explicitly include physical viscosity, but instead rely on numerical viscosity to provide the kinetic-energy dissipation. By systematically varying the numerical resolution in this approach, the energy spectrum can be extended to progressively smaller scales $\lambda \ll \delta_{L, 0}$ while maintaining the spectrum constant on larger scales. This is illustrated in Fig. 1. which shows instantaneous kinetic energy spectra in the cold nonreactive flow immediately before the flame is initiated in the computational domain in three simulations with progressively increasing resolution. Such approach allows us to vary only the intensity of the small-scale turbulent motions, and thus to investigate their effects by differentiating them from the effects of large scales. Furthermore, it shows the range of scales that must be resolved in a numerical simulation in order to capture accurately the evolution of the turbulent flame. This is particularly important in the context of high-speed turbulent reactive flows, in which it is typically impossible to resolve the Kolmogorov scale. The analysis presented in [11] demonstrated that scales $\lambda \ll \delta_{L, 0}$ have virtually no effect on the properties of the turbulent flame with the exception of the degree of flame surface wrinkling on the fuel side. This showed that the evolution of the turbulent flame can be accurately reproduced without the need to resolve scales $\lambda \ll \delta_{L, 0}$. We refer to [11] for a detailed discussion of this issue and, in particular, for a discussion of the applicability of the results to the actual stoichiometric $\mathrm{H}_{2}$-air mixture.

\subsection{Summary of simulations}

Key parameters of the simulations discussed here are summarized in Table 3 . The main difference between the three calculations is the resolution, which progressively increases from $\Delta x=\delta_{L, 0} / 8$ in $\mathrm{S} 1$ to $\Delta x=\delta_{L, 0} / 32$ in $\mathrm{S} 3$. The domain in all cases was initialized with uniform density $\rho_{0}$ and temperature $T_{0}$ (see Table 2). In S1 and S2, initial fluid velocities were set to 0 . In contrast, the velocity field in S3 was initialized with the ideal energy spectrum $\mathcal{E}(k) \propto k^{-5 / 3}$ extending from the energy injection scale $L$ to the numerical Kolmogorov scale $\eta=2 \Delta x$. This initial spectrum was normalized to ensure that at $t=0$ the total kinetic energy in the domain was equal to its predicted steady-state value [32]. In S1 and S2, the flow field was allowed to evolve for the time $t_{i g n}=3 \tau_{e d}$, and in S3 for the time $t_{i g n}=2 \tau_{e d}$, to develop the steady-state turbulent flow field. The corresponding instantaneous spectral density of the specific kinetic energy immediately prior to $t_{i g n}$ in S1-S3 is shown in Fig. 1. At the time $t_{i g n}$, a planar laminar flame with its front parallel to the $x-y$ plane 1 was initialized in the domain with the values of $\rho, T$, and $Y$ based on the exact laminar flame solution. The velocity field was not modified, thereby preserving its structure that developed during the equilibration stage.

Prior to $t_{i g n}$, all domain boundaries were periodic. At $t_{i g n}$, boundary conditions (BCs) along the left and right $z$-boundaries were switched to zero-order extrapolation in order to prevent pressure build-up inside the domain and to accommodate the resulting global fluid flow. Reflections of the acoustic perturbations caused by such BCs can be neglected in comparison with the acoustic noise generated by the turbulent flow field itself, and overall such choice of the BCs was found not to introduce any unphysical artifacts and thus not to affect the solution accuracy. Furthermore, any potential effect of the outflow BCs was minimized by the large length-to-width ratio of the computational domain, which allowed the flame brush to remain sufficiently far from the boundaries at all times [11]. Hereafter, for simplicity we refer to the moment of ignition as $t=0$.

\footnotetext{
${ }^{1}$ The longest dimension of the domain is along the $z$-axis.
} 
Energy is injected into the domain with the constant rate $\varepsilon$ per unit volume for the total duration of the simulations to provide steady driving of the turbulent flow. The value of $\varepsilon$ was chosen to produce a high-intensity turbulence field that, however, was weak enough to minimize the probability of creating weak transonic shocklets arising from the intermittency in turbulent flow. The characteristic turbulent velocities and the turbulent integral scale of the steadystate flow before the moment of ignition are listed in Table 3 . Corresponding values of the Damköhler number and the Gibson scale, given in the table, show that $D a \ll 1$ and $L_{G} \ll \delta_{L, 0}$. Prior analysis of the resulting structure of the turbulent flame demonstrated that, despite the high turbulent intensity, small-scale turbulence fails to penetrate the internal structure of the reaction zone, and the system evolves in the thin reaction zone regime [11].

The following two key global characteristics of the turbulent flame are used extensively in this work. The width of the turbulent flame brush is defined as

$$
\delta_{T}=z_{1, \max }-z_{0, \min },
$$

where $z_{0, \min }$ and $z_{1, \max }$ are defined as

$$
\begin{aligned}
& z_{0, \text { min }}=\max (z): Y(x, y, z)<0.05 \forall\left(x, y, z<z_{0, \text { min }}\right), \\
& z_{1, \text { max }}=\min (z): Y(x, y, z)>0.95 \forall\left(x, y, z>z_{1, \text { max }}\right) .
\end{aligned}
$$

In other words, $z_{0, \text { min }}$ marks the rightmost $x$-y-plane, to the left of which is pure product, while $z_{1, \text { max }}$ marks the leftmost $x$-y-plane, to the right of which is pure fuel. This is illustrated in Fig. 2] The turbulent flame speed is defined as

$$
S_{T}=\frac{\dot{m}_{R}}{\rho_{0} L^{2}}
$$

where $\dot{m}_{R}$ is the total rate of fuel consumption inside the flame brush, i.e., the total mass of reactants which is transformed to product per unit time. The detailed discussion of this choice of the definition of $S_{T}$ can be found in [11].

Kinetic energy dissipation in the turbulent flow in the domain causes gradual heating of the fuel. The resulting relative increase in the internal energy, $E_{t}$, and temperature of the nonreactive flow in one eddy turnover time, $\tau_{e d}$, can be accurately estimated as [11, 32]

$$
\frac{E_{t}-E_{t, 0}}{E_{t, 0}}=\frac{T-T_{0}}{T_{0}}=D_{K} \gamma(\gamma-1) M a_{F}^{2} .
$$

Here the constant $D_{K}=0.5$ and $E_{t, 0}$ is the thermal energy in the domain at $t=0$. Based on values of $\gamma$ from Table 2 and $M a_{F}$ from Table 3, $T$ increases by $\approx 1.8 \mathrm{~K}$ over $\tau_{e d}$. Consequently, by the end of the simulation, the fuel temperature in the domain rises from $T_{0}=293 \mathrm{~K}$ to $\approx 320-330 \mathrm{~K} 2$ As a result of such change in the thermodynamic state of fuel, the corresponding laminar flame properties also vary with time. In particular, this concerns the instantaneous thermal width, $\delta_{L}(t)$, and speed, $S_{L}(t)$, of the laminar flame, which will deviate from their initial values $\delta_{L, 0}$ and $S_{L, 0}$ corresponding to the fuel temperature $T_{0}$ and pressure $P_{0}$ (Table 2 ).

In order to determine $\delta_{L}$ and $S_{L}$ in simulations S1-S3, we found at each time $T_{\text {aver }}(t)$ and $P_{\text {aver }}(t)$ averaged over all cells located between the planes $z=z_{1, \text { max }}+L$ and $z=z_{1, \text { max }}+2 L$. This provided the average current thermodynamic state of fuel entering the flame brush. Based on $T_{\text {aver }}$ and $P_{\text {aver }}$, an exact laminar flame solution was constructed, which gave the instantaneous values of $\delta_{L}(t)$ and $S_{L}(t)$. This analysis demonstrated that in the simulations, $\delta_{L}$ decreases by $\lesssim 15 \%$. The $S_{L}$ changes more substantially increasing by $\lesssim 25 \%$. Thus, in order to consider accurately the nature of the turbulent flame speed in the high-speed turbulent reactive flows, it is important to account for the increase in the local burning speed of the flame due to the turbulent fuel heating. Consequently, in our further analysis, we relate $S_{T}$ to $S_{L}$ rather than $S_{L, 0}$.

Detailed discussion of the turbulent flame evolution in the simulations is given in [11]. Here, for completeness, we reproduce in Fig. 3 for all three calculations the time histories of $\delta_{T}$, normalized by $\delta_{L}$, as well as $S_{T}$, normalized by $S_{L}$ (cf. Fig. 4 in [11]).

Finally, Table 4 lists the time-averaged values of both $\delta_{T} / \delta_{L}$ and $S_{T} / S_{L}[11]$ as well as of other key characteristics of the turbulent flame discussed below. Table 4 also shows the corresponding order of self-convergence for each quantity listed. Since the computational cell size decreases progressively by a factor of 2 for each simulation, the

\footnotetext{
${ }^{2}$ Temperature increase occurs, on average, at constant density.
} 
order of self-convergence $O(\phi)$ of a variable $\phi$ is defined as

$$
O(\phi)=\log _{2}\left(\frac{\left|\phi_{S 1}-\phi_{S 3}\right|}{\left|\phi_{S 2}-\phi_{S 3}\right|}\right) .
$$

\section{Surface area and density of the fuel mass fraction isosurfaces}

\subsection{Isosurface area}

Fig. 4 a,b shows the evolution of the surface areas $A_{0.01}$ and $A_{0.99}$ of the isosurfaces of the fuel mass fraction $Y=0.01$ and $Y=0.99$, respectively. All isosurfaces are constructed using the "marching cubes" algorithm. These isosurfaces represent the outer boundaries that separate the flame brush from pure product and pure fuel. All values are normalized by the domain cross-section $L^{2}$ corresponding to the surface area of the planar laminar flame unperturbed by turbulence. In principle, the normalization should be performed over some average surface area of the flame brush, thus reflecting its overall large-scale shape. With sufficient accuracy, however, the turbulent flame in the system considered here can be viewed, on average, as a planar propagating front, and thus we normalize over its area $L^{2}$.

Fig. 4h shows that $A_{0.01}$ exhibits substantial variability, similar to $\delta_{T}$ and $S_{T}$ (cf. Fig. 3). There is a clear correspondence between the peaks and troughs of $A_{0.01}$ and $S_{T}$ and, to a lesser extent, $\delta_{T}$. Moreover, in a manner similar to $\delta_{T}$ and $S_{T}, A_{0.01}$ varies less with increasing resolution, and it appears on average to have converged.

Such correlation between $A_{0.01}$ and $S_{T}$ reflects the evolutionary cycle of the flame brush, discussed in [11]. In the turbulent flame, which on average is in a steady state, the influx of fresh fuel and the rate of its consumption never perfectly balance each other. Instead, periodically either one or the other process dominates. In particular, an increase in the flame surface area inside the brush, and the associated increase in $\delta_{T}$, leads to a higher $S_{T}$. This causes rapid consumption of fuel inside the brush, decreasing both the flame surface area and the overall width of the flame brush. The result is a slower, less convolved flame, which is thus more susceptible to the action of turbulence. This leads to increased folding and stretching of the flame, and the cycle repeats.

The behavior of $A_{0.99}$ is pronouncedly different. There appears to be no correlation between variations in $A_{0.99}$ and either $\delta_{T}$ or $S_{T}$. Moreover, with increasing resolution, the magnitude of the variations increases, and there is no evidence of convergence of the growing average values of $A_{0.99}$. We will consider the correlation between $A_{Y}$ and both $S_{T}$ and $\delta_{T}$ in a more quantitative form in $\S 4$

These conclusions regarding the change of $A_{0.01}$ and $A_{0.99}$ with resolution are supported by Fig. 4t, which shows the full normalized time-averaged distributions $\bar{A}_{Y} / L^{2}$ for all values of $Y$. In simulation S1, $\bar{A}_{0.01}$ and $\bar{A}_{0.99}$ are nearly equal. With increasing resolution, however, they diverge to the point that, in $\mathrm{S} 3$, there is almost a factor of 2 difference between them. Values of $\bar{A}_{0.01}$ steadily decrease with resolution and they indeed demonstrate convergence. At the same time, $\bar{A}_{0.99}$ increases with resolution, and the difference between S2 and S3 is only marginally smaller than between $\mathrm{S} 1$ and $\mathrm{S} 2$.

This behavior is part of a broader qualitative change in the overall shape of the distribution of $\bar{A}_{Y}$ that occurs around $Y \approx 0.5$, i.e., at the boundary of the reaction and preheat zones. The lower resolution of S1 suppresses smallscale turbulent motions, which, in turn, causes less wrinkling of the isosurfaces in the preheat zone. As a result, the flame-brush surface appears similar both on the product and fuel sides (cf. Fig. 3 in [11]). With higher resolution, however, isosurface wrinkling becomes more pronounced with increasing distance from the reaction zone, and the distribution of $\bar{A}_{Y}$ develops a distinctive inverted-S shape, as seen in Fig. 4t. The consequence of this was observed in [11], which reported a much more highly convolved flame-brush surface on the fuel side in calculations S2 and S3. We will discuss the role of small-scale turbulence in further detail in $\$ 3.4$

Profiles of $\bar{A}_{Y}$ in S2 and S3 are closer to each other in the reaction zone than in the preheat zone (Fig.44). Table 4 shows that in the region of peak reaction rate, i.e., at $Y \approx 0.15, \bar{A}_{Y} / L^{2}$ exhibits the $3^{\text {rd }}$-order convergence. This is substantially faster than what would be expected for an overall $2^{\text {nd }}$-order-accurate code. Moreover, in all three calculations, $\bar{A}_{Y}$ varies the slowest inside the reaction zone, i.e., for $Y \approx 0.15-0.6$. This shows that isosurfaces of $Y$, on average, follow each other relatively closely within the reaction zone, which thus can be viewed as being uniformly folded and stretched by turbulence as a coherent structure. This is consistent with the very low variability in the reaction zone of the instantaneous profiles of $Y$ and $T$, which represent the internal flame structure [11]. 


\subsection{Isosurface density}

The analysis of the isosurface area distribution does not show how the flame is organized inside the flame brush and, in particular, how tightly it is packed. More specifically, the question arises whether variations in the isosurface

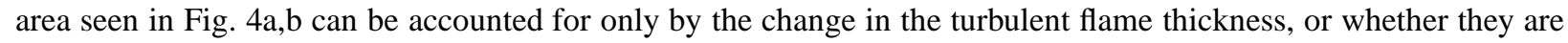
also related to how the flame is folded inside the flame brush.

Consider the surface density of the fuel mass fraction isosurfaces, defined as the isosurface area for a given $Y$ normalized by the effective flame-brush volume,

$$
\Sigma_{Y}=\frac{A_{Y}}{\delta_{T} L^{2}} .
$$

A detailed discussion of this definition of $\Sigma_{Y}$ and, in particular, of the choice of the uniform normalization by the full flame-brush volume is given in Appendix A

The evolution of $\Sigma_{0.01}$ and $\Sigma_{0.99}$ is shown in Fig. 4te,d. The overall trends are similar to those exhibited by $A_{0.01}$ and $A_{0.99}$. In particular, $\Sigma_{Y}$ is lower on the product side of the flame. Similar to $A_{Y}, \Sigma_{Y}$ rapidly converges with resolution at lower values of $Y$ and shows virtually no convergence in the preheat zone. Most important, there is substantial variability in $\Sigma_{Y}$, especially on the fuel side, where $\Sigma_{Y}$ changes by more than a factor of 3 in the highest resolution case. This tighter packing of the isosurfaces, which occurs periodically in the course of system evolution, suggests that the increase in $A_{Y}$ is associated not only with the increase of $\delta_{T}$ but also of $\Sigma_{Y}$.

Fig. 4f shows the time-averaged distributions of $\Sigma_{Y}$ in simulations S1-S3. Comparison with Fig. 4e demonstrates that both $\bar{A}_{Y} / L^{2}$ and $\bar{\Sigma}_{Y}$ show remarkably similar behavior. The time-averaged values of $\bar{\Sigma}_{Y}$ are also not constant, but, instead, for sufficiently high numerical resolution, they progressively increase through the flame interior from its product side to the fuel side. The resulting inverted-S shape of the profiles is very similar to that of $\bar{A}_{Y}$ with both $\bar{A}_{Y}$ and $\bar{\Sigma}_{Y}$ at the two extreme values of $Y(Y=0.01$ and 0.99$)$ differing in S3 by almost a factor of two. Furthermore, similar to $\bar{A}_{Y}, \bar{\Sigma}_{Y}$ varies the least in the interior of the reaction zone, i.e., for $Y \approx 0.15-0.6$, while outside this region it shows a strong dependence on $Y$. Just outside the reaction zone, individual profiles begin to diverge from each other, with the variation between them becoming progressively larger for higher values of $Y$.

In the reaction zone, values of $\bar{\Sigma}_{Y}$ for all three calculations are much closer than those of $\bar{A}_{Y}$. In fact, Table 4 shows that $\bar{\Sigma}_{Y}$ exhibits $4^{\text {th }}$-order convergence compared with $3^{\text {rd }}$-order convergence for $\bar{A}_{Y}$. As a result, values of $\bar{\Sigma}_{Y}$ are virtually identical for $Y \approx 0.01-0.4$ in S2 and S3. On the other hand, in the preheat zone, the profiles of $\bar{\Sigma}_{Y}$ diverge more than in the case of $\bar{A}_{Y}$. This shows that the effects of small-scale turbulent motions on the reaction and the preheat zones are, in fact, even more disparate in terms of their ability to provide tight folding of the isosurfaces, compared with their ability to increase the isosurface area.

Flame surface density is a quantity used both in experimental and theoretical combustion research. In particular, $\Sigma_{\max }$ is defined as the surface density in the region of the flame brush with mean reactedness $\bar{c}=0.5$, where $c=$ $\left(T-T_{0}\right) /\left(T_{P}-T_{0}\right)$ and $T_{P}$ is the post-flame temperature. It can then be shown that [3]

$$
\Sigma_{\max }=\frac{A_{0.5}^{\prime}}{\delta_{T} A_{L}^{\prime}}
$$

where $A_{0.5}^{\prime}$ is the flame surface area based on $\bar{c}=0.5$, and $A_{L}^{\prime}$ represents the average area of the flame brush. In a system described by the one-step Arrhenius kinetics, $\bar{c}=0.5$ corresponds to $Y=0.5$. Therefore, $A_{0.5}^{\prime} \equiv A_{0.5}$. At the same time, in a planar brush $A_{L}^{\prime}=L^{2}$, as was discussed in $\S 3.1$ Thus, $\Sigma_{\max }$ is equivalent to $\Sigma_{0.5}$ as given by eq. (14). It is reasonable then to compare values of $\Sigma_{0.5}$ in simulations S1-S3 with those of $\Sigma_{\max }$ obtained in other experimental and numerical settings. In particular, in $\mathrm{S} 3, \bar{\Sigma}_{0.5}=0.73 \mathrm{~mm}^{-1}$. This is comparable to the range of values of $\Sigma_{\max } \approx 0.12-0.6 \mathrm{~mm}^{-1}$ obtained in a number of experimental studies using a wide variety of flame configurations (see [3] for a summary table). The fact that the value in S3 is somewhat larger is not surprising, given that the turbulent intensity in it is substantially higher than in the experiments.

Overall, however, the traditional definition of $\Sigma[3]$ is not equivalent to the definition of $\Sigma_{Y}$ used here unless $Y=0.5$. Therefore, unlike $\Sigma_{\max }, \Sigma_{0.5}$ is not necessarily the maximum value of $\Sigma_{Y}$ for all values of $Y$. Fig. [4 shows that while in S1 $\bar{\Sigma}_{0.5}$ is indeed approximately the largest value, this is not the case in S2 and S3. Nonetheless, in all three calculations, $\bar{\Sigma}_{0.5}$ is representative of the values of $\bar{\Sigma}_{Y}$ throughout the reaction zone with the difference between 
$\bar{\Sigma}_{0.5}$ and $\bar{\Sigma}_{0.15}$ being $\approx 6-9 \%$ in the higher-resolution cases.

\subsection{Relation between $A_{Y}$ and $\Sigma_{Y}$}

Fig. 5 shows the relation between the isosurface area and density in three key regions of the flame: the peak reaction rate $(Y=0.15)$, the boundary of the preheat and reaction zones $(Y=0.5)$, and the coldest region of the preheat zone $(Y=0.95)$. A pronounced linear correlation between $A_{Y} / L^{2}$ and $\Sigma_{Y}$ exists throughout the flame interior. This demonstrates that at all values of $Y$, an increase of $A_{Y}$ is greatly influenced by the associated tighter packing of the isosurfaces inside the flame brush.

With increasing $Y$ the average $\bar{A}_{Y} / L^{2}$ and $\bar{\Sigma}_{Y}$ become larger, as was seen in Fig. 44,f, due to the overall shift of the distributions toward larger values of both $A_{Y} / L^{2}$ and $\Sigma_{Y}$. Note also a slight increase both in the scatter of the distribution and the slope of its least squares fit at higher $Y$. Thus, isosurfaces of higher fuel-mass fractions are indeed consistently more tightly packed than at lower $Y$. This is in agreement with the observation that the flame surface is wrinkled on finer scales on the fuel side than on the product side [11].

\subsection{Distributions of $\bar{A}_{Y}$ and $\bar{\Sigma}_{Y}$ and the effects of small-scale turbulence}

How do these results concerning the distributions of $\bar{A}_{Y}$ and $\bar{\Sigma}_{Y}$, shown in Fig.44,f, relate to previous conclusions [11] regarding the inability of the turbulent cascade to penetrate the flame interior and the role of small-scale turbulent motions? As was discussed in $\S 2.1$ (also see [11] for further details), the progressive increase in resolution from S1 to $\mathrm{S} 3$ extends the turbulent cascade to smaller scales, and this leads to a substantial increase in the energy of turbulent motions on scales $\lambda<\delta_{L}$ in nonreactive turbulence. This increase in resolution was found to cause the flame surface on the fuel side to be wrinkled on progressively finer scales, while remaining virtually unchanged on the product side. Furthermore, it was determined that the deviation of the internal turbulent flame structure from that of the planar laminar flame increases with decreasing temperature. These two results showed that the effects of small-scale turbulent motions are most pronounced in the coldest parts of the preheat zone. With increasing temperature, these effects diminish and they completely disappear once the reaction rate becomes significant [11].

The results presented above are consistent with this picture. The steady growth of $\bar{A}_{Y} / L^{2}$ and $\bar{\Sigma}_{Y}$ on the fuel side of the preheat zone with increasing resolution shows that more intense small-scale motions are indeed present there, folding isosurfaces on progressively finer scales. It must be emphasized that the change in $\bar{A}_{Y}$ alone does not necessarily mean that it is caused by finer wrinkling on smaller scales. Only when viewed in conjunction with the surface density, which shows a very similar dependence on $Y$, does such increase in $\bar{A}_{Y}$ serve as a strong indication of the small-scale wrinkling.

With increasing temperature, not only do $\bar{A}_{Y} / L^{2}$ and $\bar{\Sigma}_{Y}$ decrease rapidly, but also profiles for S2 and S3 begin to approach each other. This means that the main difference between these two simulations, namely the presence of more energetic small-scale turbulence in $\mathrm{S} 3$, is being eliminated and, thereby, motions on scales $\lambda<\delta_{L}$ are gradually suppressed. Since it is these small scales that enhance the diffusive transport, which in turn broadens the preheat zone, their suppression causes the internal flame structure to approach that of the planar laminar flame [11].

As the reaction rate becomes substantial at $Y \lesssim 0.5$, both $\bar{A}_{Y} / L^{2}$ and $\bar{\Sigma}_{Y}$ become similar in S2 and S3. This suggests that, at this point, only scales $\lambda \gtrsim \delta_{L}$ remain energetic. Indeed, these scales are originally the same in both calculations (cf. Fig. 11). Consequently, they generate similar isosurface areas and densities. Since these scales cannot support small-scale diffusive transport, any broadening of the internal flame structure effectively disappears at $Y \lesssim 0.5$ [11]. Further decrease in $\bar{A}_{Y} / L^{2}$ and $\bar{\Sigma}_{Y}$ is the consequence of the continued suppression of the progressively larger scales $\lambda>\delta_{L}$.

There is one important distinction between the distributions of $\bar{A}_{Y} / L^{2}$ and $\bar{\Sigma}_{Y}$ and the time-averaged profiles of $Y$ and $T$, which represent the internal flame structure [11]. The $Y$ and $T$ profiles showed very little variation between simulations S1-S3 not only in the reaction zone, but also in the preheat zone. This is in contrast with the distributions $\bar{A}_{Y} / L^{2}$ and $\bar{\Sigma}_{Y}$, which differ substantially in the preheat zone even between S2 and S3. Such discrepancy suggests that small scales, $\lambda<\delta_{L}$, contribute differently to the turbulent diffusive transport and to the wrinkling of the isosurfaces. The former is primarily governed by the largest of these small scales, i.e., scales not much smaller than $\delta_{L}$, since these scales are associated with the highest velocities. The energy contained on these scales is close in all three simulations and, thus, they produce the same structure of the broadened preheat zone. At the same time, all scales smaller than $\delta_{L}$ contribute to the isosurface wrinkling, and the different energy content of these scales causes the distributions of $\bar{A}_{Y} / L^{2}$ and $\bar{\Sigma}_{Y}$ to differ substantially in the preheat zone. 
Qualitatively, both the internal flame structure described in [11] and the distributions of $\bar{A}_{Y}$ and $\bar{\Sigma}_{Y}$ presented here create a consistent picture of the transformation that turbulence undergoes as it passes through the flame. Changes in the energy budget between different scales, which are most certainly accompanied by the development of both anisotropy and inhomogeneity of the velocity field, are complex. While the evidence presented here and in [11] provide hints about the nature of this transformation, the details remain unknown. For instance, are the energy release and the resulting fluid expansion the only effects responsible for altering the turbulent field and redistributing energy between different scales? What are the relative contributions of various small scales to the increase in $A_{Y}$ and $\Sigma_{Y}$ ? How does the shift in balance between small and large scales with increasing temperature change at different turbulent intensities? All of these questions need to be addressed in future studies.

\section{What is the flame surface area?}

In the wrinkled and corrugated flamelet regimes, in which eq. (1) is typically applicable, the flow is laminar on scales $\lambda \lesssim \delta_{L}$. As a result, isosurfaces of different values of $Y$ are parallel to each other, the flame is folded by turbulence as a coherent structure, and, therefore, it has a well-defined surface area $A_{T}$. Consequently, the stretch factor $I$ has a unique value at each moment in time, and so it directly shows how much of the increase of $S_{T}$ can be ascribed to the increase of $A_{T}$.

In contrast, in the thin reaction zone regime discussed here, results presented in $\S 3$ demonstrate that the flame, folded inside the flame brush by high-intensity turbulence, has a complex internal structure. The various parts of the flame have distinctly different responses of its various parts to the action of turbulence. Consider, by analogy with eq. (1), the factor $I_{Y}$, which relates the increase of the turbulent flame speed relative to its planar laminar value to the increase of $A_{Y}$ rather than $A_{T}$,

$$
\frac{S_{T}}{S_{L}}=I_{Y} \frac{A_{Y}}{L^{2}}
$$

In this case, unlike $I, I_{Y}$ is a function of $Y$. In particular, based on the data shown in Fig. 4, $I_{Y}$ can vary by as much as a factor of 4 depending on the choice of $Y$. As a result, values of $I_{Y}$ close to and substantially larger than unity can be found in different regions of the flame.

This shows that before we can answer the question whether the observed $S_{T}$ can be accounted for by the increase in the flame surface area, we must first determine what this area is in such high-speed turbulent flows, and whether such a concept is even applicable in this regime. In particular, what value of $Y$ fully and accurately represents the overall behavior of the turbulent flame? We address these questions by considering the correlation of $A_{Y}$ with two global properties of the turbulent flame, its speed and width.

\subsection{Relation between $A_{Y}$ and $S_{T}$}

Fig. 6 shows correlations between $S_{T} / S_{L}$ and $A_{Y} / L^{2}$ calculated for the same three representative values of $Y$ as in Fig. 5, namely $Y=0.15$, corresponding to the region of peak reaction rate, $Y=0.5$, corresponding to the boundary between the reaction and the preheat zones, and $Y=0.95$, corresponding to the coldest part of the preheat zone. Left panels of Fig. 6 show the time-evolution of all quantities, and the right panels show the corresponding correlation scatter plots.

Since both the local flame speed and the induction time of the unburned fuel have finite values, there must exist a delay in the response of $S_{T}$ to the changes in the flame configuration, represented by the areas of its isosurfaces. Therefore, the degree of correlation between $S_{T}$ and $A_{Y}$ is a function of a time lag, $\Delta t$, between these two quantities. For each value of $Y$ in Fig. 6, we determined the time lag, $\Delta t_{c}$, that produced the best correlation. This was done by directly calculating the cross-correlation between $S_{T}(t) / S_{L}$ and $A_{Y}(t) / L^{2}$ and finding its maximum. The value obtained for $\Delta t_{c}$ was verified by determining the least squares fit for the distribution of $S_{T}(t) / S_{L}$ vs. $A_{Y}\left(t-\Delta t^{\prime}\right) / L^{2}$ and by finding $\Delta t_{c}^{\prime}$ that maximized the slope of the fit and minimized its residuals. The values of $A_{Y} / L^{2}$ in Fig. 6 were then shifted in time with respect to $S_{T} / S_{L}$ by the corresponding time lag $\Delta t_{c}$ given in the lower right corner of the panels (a), (c), and (e).

The first key conclusion emerging from Fig. 6 is that $S_{T}$ and $A_{Y}$ become progressively less correlated with increasing $Y$. While the correlation is pronounced throughout the reaction zone and is exceptionally strong near the peak reaction rate, $S_{T}$ and $A_{Y}$ are only very weakly correlated in the colder parts of the preheat zone. Note that panels 
(b), (d), and (f) have the same scale, which shows the relative increase in the scatter of the distribution and its overall shift to higher values of $A_{Y}$ in the preheat zone.

The time delays that give the best correlation between the two quantities are positive, and thus they show that $S_{T}$ does indeed lag behind $A_{Y}$. Moreover, the magnitude of $\Delta t_{c}$ increases with increasing $Y$. Qualitatively, this agrees with the fact that $S_{T}$ at any given moment is primarily determined by the region of the highest reaction rate, which corresponds to the values of $Y$ close to 0.15 . Consequently, the time delay between $S_{T}$ and the area of the isosurface representing the peak reaction rate is close to zero, namely $0.04 \tau_{e d}$. Since turbulence reorganizes the flame on a timescale $\sim \tau_{e d}$, such a small lag is too short for the flame structure to change in any significant way. Therefore, the correlation between $A_{0.15}$ and $S_{T}$ is very strong.

At the same time, since the reaction rate is fairly low at $Y=0.5$, the contribution of this region to the overall turbulent flame speed is small. Burning still needs to accelerate substantially in this area in order for it to become the new region of peak reaction rate, which will then predominantly determine the magnitude of $S_{T}$. The time required for this to occur results in a longer time delay, namely $0.11 \tau_{e d}$. During this time, however, turbulence is able to change the flame structure more strongly than in the case of $Y=0.15$. As a result, the degree of correlation between $A_{0.5}$ and $S_{T}$ decreases.

In the coldest regions of the preheat zone, there is no correlation between $S_{T}$ and $A_{0.95}$ at small values of $\Delta t_{c}$. We found evidence of weak correlation for a much larger time delay $\Delta t_{c}=0.61 \tau_{e d}$. This value, however, is comparable to the time during which the turbulence completely reorganizes the structure of the turbulent flame. Therefore, by the time burning reaches the reactants in the outer regions of the preheat zone, the connection between their original distribution and the resulting turbulent flame speed is significantly disrupted.

We also found weak correlation between $A_{0.95}$ and $A_{0.15}$ with the time lag for $A_{0.95}$ being $\Delta t_{c}=0.56 \tau_{e d}$ and the slope of the least squares fit of 0.49073 This weak correlation between $A_{0.15}$ and $A_{0.95}$ is consistent with a similarly weak correlation between $S_{T}$ and $A_{0.95}$ found at a comparable time lag. In particular, it reflects the fact that after the time $\approx 0.6 \tau_{e d}$, burning reaches the outer part of the preheat zone. As a result, the former $Y=0.95$ region becomes the new $Y=0.15$ region and thus the new site of the peak reaction rate which now determines $S_{T}$. Substantial change in the flame-brush structure during this time, however, again leads only to weak correlation between $A_{0.15}$ and $A_{0.95}$.

In order to verify this physical meaning of the obtained time delays, values of $\Delta t_{c}$ can be compared with the characteristic induction time, $\tau_{\text {ind }}$, in the planar laminar flame. The $\tau_{\text {ind }}$ is the time for a fluid element to reach the peak reaction rate starting at some initial temperature or, equivalently, fuel mass fraction. In reactive mixtures with large activation energies, such as $\mathrm{H}_{2}$-air considered here, $\tau_{\text {ind }}$ is also approximately the time necessary to reach the post-flame temperature, $T_{P}[36]$.

The $\tau_{\text {ind }}$ can be calculated using the velocity distribution $U_{L}(x)$ in the exact planar laminar flame solution as

$$
\tau_{\text {ind }}\left(Y^{\prime}\right)=\int_{x\left(Y^{\prime}\right)}^{x\left(Y^{*}\right)} \frac{d x}{U_{L}(x)-U_{\infty}} .
$$

Here $U_{\infty}$ is the fuel velocity at infinity, $Y^{\prime}$ is the initial fuel mass fraction of the fluid element, and $Y^{*}$ is the fuel mass fraction corresponding to the peak reaction rate. Instead, to maintain consistency with the numerical simulations, we computed $\tau_{\text {ind }}$ directly by solving the full time-dependent reactive Navier-Stokes equations (3)-(8) on a uniform 1D grid with the same numerical integration method used for S1-S3 (\$2.1). The flame was initialized as a discontinuity with the fuel and product temperature and density equal to $T_{0}, \rho_{0}$ and $T_{P, 0}, \rho_{P, 0}$, respectively, given in Table 2 . Constant pressure $P_{0}$ and zero velocity were set throughout the domain. We introduced an additional advection equation describing a scalar $\xi$ that was not subject to diffusion or reactions and was only advected with the flow. After the steady-state structure of the flame had been established in the computational domain, $\xi$ was set to unity in one cell corresponding to the desired initial $T^{\prime}$ or, equivalently, $Y^{\prime}$, and to zero everywhere else. As the system evolved, the fluid element marked with the scalar $\xi$ moved through the flame and burned. The time it took this fluid element to reach peak reaction rate, i.e., $Y=0.15$, gave $\tau_{\text {ind }}\left(Y^{\prime}\right) 4$

\footnotetext{
${ }^{3}$ In contrast, we were unable to find any statistically significant correlation or anticorrelation between $\Sigma_{0.15}$ and $\Sigma_{0.95}$ at any time lag.

${ }^{4}$ The distribution of $\xi$ would spread with time over several cells due to numerical diffusion. To compensate for this, we used very high resolution, namely $\Delta x=\delta_{L, 0} / 128$, which is much larger than the resolution necessary to obtain an accurate laminar flame solution, i.e., typically $\Delta x=\delta_{L, 0} / 4$ [32]. As a result, the distribution of $\xi$ was found to spread over the range of values of $Y$ with the full width at half maximum equal to $\Delta Y \approx 0.05$. We
} 
Using this procedure, we found that for a planar laminar flame,

$$
\begin{aligned}
& \tau_{\text {ind }} \approx 0.27 \tau_{e d} \approx 2.46 \Delta t_{c}, \text { for } Y=0.5, \\
& \tau_{\text {ind }} \approx 1.29 \tau_{\text {ed }} \approx 2.12 \Delta t_{c}, \text { for } Y=0.95 .
\end{aligned}
$$

When turbulent heating of the fuel is taken into account (see $\$ 2.2$ ), i.e., when the fuel temperature in this calculation is increased to $320 \mathrm{~K}$ from $T_{0}=293 \mathrm{~K}$ while maintaining constant density $\rho_{0}$, these values of $\tau_{\text {ind }}$ decrease by $\approx 20-25 \%$. Further details and, in particular, the full distributions of $\tau_{\text {ind }}$ throughout the flame for these two fuel temperatures, can be found in Appendix B

The calculated time lags are, therefore, within a factor of $\approx 2$ of the corresponding values of $\tau_{\text {ind }}$, which, given the statistical nature of $\Delta t_{c}$, is reasonable agreement. This shows that the correlation time delays for the distributions of $S_{T} / S_{L}$ and $A_{Y} / L^{2}$ in the turbulent flame can indeed be associated with the induction times necessary for the burning to accelerate in regions with higher reactant concentrations.

The values of $\Delta t_{c}$, however, are substantially lower than the characteristic propagation time of the laminar flame,

$$
\tau_{\delta}=\frac{\delta_{L, 0}}{S_{L, 0}}=\frac{30}{8} \frac{L}{U}=3.75 \tau_{e d}
$$

where we used the values of $L$ and $U$ given in Table 3 . Even for $Y=0.95, \Delta t_{c}=0.61 \tau_{e d} \approx(1 / 6) \tau_{\delta}$. Furthermore, this $\Delta t_{c}$ is an order of magnitude smaller than the time $1.67 \delta_{L, 0} / S_{L, 0} \approx 6.3 \tau_{e d}$, which is necessary for the flame to propagate over the characteristic distance $1.67 \delta_{L, 0}$ separating $Y=0.95$ and $Y=0.15$ in the laminar flame structure (cf. Fig. 7 in [11]).

A good analytical estimate for $\Delta t_{c}$ throughout the reaction zone can be obtained using the expression for the adiabatic induction time [36],

$$
\tau_{\text {ind }}^{a}\left(Y^{\prime}\right)=\left(\frac{C_{p} T^{\prime}}{B q \rho^{\prime} Y^{\prime}}\right)\left(\frac{R T^{\prime}}{Q}\right) \exp \left(\frac{Q}{R T^{\prime}}\right),
$$

where $T^{\prime}$ and $\rho^{\prime}$ for a given $Y^{\prime}$ are determined based on the exact planar laminar flame solution, and the values of $B$, $q$, and $Q$ are given in Table 2 . In particular, at the fuel temperature $T_{0}$ and density $\rho_{0}$,

$$
\begin{aligned}
& \tau_{\text {ind }}^{a} \approx 0.05 \tau_{e d} \approx 1.25 \Delta t_{c}, \text { for } Y=0.15, \\
& \tau_{\text {ind }}^{a} \approx 0.21 \tau_{e d} \approx 1.91 \Delta t_{c}, \text { for } Y=0.5 .
\end{aligned}
$$

Using the specific heat at constant volume $C_{v}=R / M(\gamma-1)$ instead of $C_{p}$ in eq. (20), i.e., assuming that burning occurs at constant volume rather than at constant pressure, would decrease the values of $\tau_{\text {ind }}^{a}$ by $\approx 15 \%$ to $0.04 \tau_{\text {ed }}$ and $0.18 \tau_{e d}$, respectively. This shows that, even though $\tau_{\text {ind }}^{a}$ is typically applicable in the context of an autoignition process rather than propagation of a flame, for $Y=0.5$ it provides a somewhat better approximation to the calculated value of $\Delta t_{c}$ than $\tau_{\text {ind }}$ given in eq. (18). Further details, including the effects of turbulent fuel heating on $\tau_{\text {ind }}^{a}$, are given in Appendix B.

Finally, in addition to the strong correlation between the values of $S_{T}$ and $A_{0.15}$, the distribution shown in Fig. $6 \mathrm{~b}$ has another important property that is not present at higher values of $Y$. The linear least squares fit for the case $Y=0.15$, shown with a solid line in Fig. 6b, has the form

$$
\frac{S_{T}}{S_{L}}=1.17 \frac{A_{0.15}}{L^{2}}-0.08
$$

For $A_{0.15} / L^{2}=1$, this expression gives $S_{T} / S_{L}=1.09$. This demonstrates that as the flame becomes less convolved, i.e., as $A_{0.15} / L^{2} \rightarrow 1$, it behaves progressively more like a planar laminar flame which is manifested in $S_{T} \rightarrow S_{L}$. Linear least squares fits for the two other values of $Y$, shown in Fig. 6 d,f, do not recover the value of $S_{T} / S_{L}=1$ as $A_{Y} / L^{2} \rightarrow 1$ 


\subsection{Relation between $A_{Y}$ and $\delta_{T}$}

Next consider the correlation of $A_{Y}$ with the second key global characteristic of the turbulent flame, namely its width, $\delta_{T}$. Fig. 7 shows the correlation between $A_{Y} / L^{2}$ and $\delta_{T} / L$ for the same three values of $Y$ as in Fig. 65 The distribution of $\delta_{T} / L$ as a function of $A_{Y} / L^{2}$ shows the same trend observed for $S_{T} / S_{L}$. In particular, the correlation between the two quantities is also the strongest for $Y=0.15$ and decreases with increasing $Y$. The values of $\delta_{T} / L$ and $A_{0.95}$ appear to be almost completely uncorrelated. Note, however, that the correlation in the reaction zone, and, in particular, in the region of peak reaction rate, is weaker for $\delta_{T}$ than $S_{T}$, with the distributions of $\delta_{T}$ having a much larger scatter.

In order to illustrate the relation between the three key quantities characterizing the instantaneous flame-brush structure, namely $A_{Y}, \delta_{T}$, and $\Sigma_{Y}$, each data point in Fig. 7 is colored according to the corresponding value of $\Sigma_{Y}$ (cf. Fig. 5). At lower $Y$, the increase of the isosurface area is associated both with the increase in the overall extent of the flame brush and with tighter packing of the isosurfaces. With increasing $Y$, however, the correlation of $A_{Y} / L^{2}$ with $\delta_{T} / L$ decreases while remaining quite pronounced with $\Sigma_{Y}$. This shows that the area of the isosurfaces of higher $Y$ changes primarily only due to their wrinkling on progressively smaller scales, as discussed in $\S$ (also see [11]). Indeed, $\delta_{T}$ is determined predominantly by the flame folding on the largest scales. Consequently, the weak correlation between $A_{Y}$ and $\delta_{T}$ at high $Y$ demonstrates that the increase of the areas of these isosurfaces cannot be associated with such large-scale wrinkling. Instead, this increase must be translated primarily into tighter packing of the isosurfaces causing $A_{Y}$ to be correlated with $\Sigma_{Y}$.

\section{What determines $S_{T}$ in the thin reaction zone regime?}

\subsection{Can $S_{T}$ be explained by the increase in the flame surface area?}

The strong correlation of $A_{0.15}$ with $S_{T}$ and $\delta_{T}$, which represent both the global energetics and the global structure of the turbulent flame, show that, in the thin reaction zone regime, isosurfaces of $Y$ close to the peak reaction rate accurately characterize the overall evolution of the turbulent flame. Consequently, for the reactive mixture considered here, the flame surface area, $A_{T}$, and density, $\Sigma_{T}$, are represented by the isosurface of $Y=0.15$.

This conclusion is further supported by the limiting behavior of the distribution of $S_{T} / S_{L}$, which approaches unity as $A_{0.15} / L^{2} \rightarrow 1$. In contrast, at $Y=0.5, S_{T} / S_{L}$ becomes progressively less than $A_{0.5} / L^{2}$ at smaller isosurface areas. Furthermore, at $Y=0.95$, almost all values of $S_{T} / S_{L}$ lie below the $S_{T} / S_{L}=A_{0.95} / L^{2}$ line, and they become almost half of $A_{0.95} / L^{2}$ at larger isosurface areas. In $L e=1$ reactive mixtures, there are no mechanisms that can lower the local flame speed below $S_{L}$, which would be necessary to produce values of $S_{T} / S_{L}$ lower than the increase of the flame surface area. Consequently, such properties of the distributions of $S_{T} / S_{L}$ confirm that isosurfaces of higher $Y$ cannot represent the flame surface.

With this definition of the surface area of the turbulent flame, we can now revisit the question of the relation between $A_{T}$ and the speed of the turbulent flame in the thin reaction zone regime. This relation can be represented by eq. (16) in which $Y=0.15$, similarly to eq. (1) applicable in the wrinkled and corrugated flamelet regimes 6 Fig. 8 shows the ratio $I_{0.15}=\left(S_{T} / S_{L}\right) /\left(A_{0.15} / L^{2}\right)$ for the distribution given in Fig. $6 \mathrm{~b}$. Time-averaged values $\bar{I}_{0.15}=$ $\overline{\left(S_{T} / S_{L}\right) /\left(A_{0.15} / L^{2}\right)}$ for all three calculations are listed in Table 47 In particular, $\bar{I}_{0.15}=1.14$ in S3, and this value can be viewed as converged to within a few percent with a faster than linear rate of convergence.

These results lead to the following two key conclusions:

1. In the thin reaction zone regime, the instantaneous turbulent flame speed is also primarily determined by the increase of the flame surface area.

\footnotetext{
${ }^{5}$ Since both $A_{Y}$ and $\delta_{T}$ represent the same instantaneous configuration of the flame, no time delay between them is expected. Consequently, no time shift was applied to either quantity in Fig. 7 As a check, however, we verified that indeed the cross-correlation had its maximum at the zero time lag.

${ }^{6}$ In light of the fact that $S_{T}$ and $A_{Y}$ are best correlated when $A_{Y}$ is shifted in time with respect to $S_{T}$, the question arises whether such a time shift should also be applied in eq. (16). In principle, an argument could be made that, due to the inherent delay in the response of $S_{T}$ to the changes in the flame structure, the current value of $S_{T}$ is the result of $A_{Y}$ that existed earlier in time and, thus, this earlier value must be used to assess the relation between $S_{T}$ and $A_{Y}$. At the same time, as was shown, the time lag $\Delta t_{c}$ is extremely small for $Y=0.15$. During this time, the change in the value of $A_{Y}$ is negligible and applying the time shift in eq. (16) does not cause any appreciable change in the result.

${ }^{7}$ Using instead the expression $\bar{I}_{0.15}=\left(\overline{S_{T} / S_{L}}\right) /\left(\bar{A}_{0.15} / L^{2}\right)$ gives virtually the same result.
} 
2. In addition, in the course of system evolution, $S_{T}$ periodically exhibits an exaggerated response to the increase in $A_{T}$. For the system size and turbulence intensity considered here, this excess burning velocity can become as high as $30 \%$ of $A_{T} / L^{2}$.

This exaggerated response in simulation S3 is represented by values of $I_{0.15}>1 \mathrm{in} \mathrm{Fig.} 8$ and is illustrated in Fig. 61, b as the shaded gray area. Such a substantial deviation of $I_{0.15}$ from unity brings up the following question: what mechanism is responsible for raising $S_{T}$ beyond what can be attributed to the increase in $A_{T}$ ?

\subsection{Potential causes of the excess values of $S_{T}$}

As was discussed in § [1, Damköhler's concept [1] implies that the burning velocity of a turbulent flame in the presence of sufficiently intense turbulence is determined by two processes: wrinkling of the flame surface by largescale turbulent motions increasing $A_{T}$, and the enhancement of the local flame speed $S_{n}$ by the small-scale turbulence providing stronger diffusive transport. While the simulations show that the effect of large-scale turbulence is indeed present and has a dominant effect on $S_{T}$, can the observed excess values of $S_{T}$ be ascribed to the action of small-scale motions?

Consider the following important characteristic exhibited by the distribution of $I_{0.15}$. Along with $I_{0.15}$ substantially larger than one, the system also periodically develops values of $I_{0.15}$ within only a few percent of unity, which is comparable to the uncertainty in estimating the instantaneous $A_{0.15}$ and, thus, $I_{0.15}$ (see also Fig. 6a). This shows that at those instances when $I_{0.15} \approx 1, S_{n}$ at all points of the flame surface must be almost identical to $S_{L}$. Consequently, the small-scale turbulence entering the flame, if it were directly determining $S_{n}$, would have to periodically undergo transitions between almost complete suppression, when $I_{0.15} \approx 1$ and $S_{n} \approx S_{L}$, and significant enhancement, when $I_{0.15}$ becomes as high as 1.3 .

While we are unaware of any potential mechanisms that could cause such periodic complete suppression of smallscale turbulence in the cold fuel, our analysis also does not show any changes in the state of turbulence that could serve as a manifestation of such suppression. In order to determine whether there exists a connection between the instantaneous turbulent field in the domain and the resulting burning speed, we have analyzed the correlation of several turbulence characteristics with both $S_{T}$ and $I_{0.15}$. In particular, we considered the total r.m.s. velocity in the domain. Furthermore, to characterize turbulence inside the flame brush and in the fuel entering it, we also considered individual components of velocity, $u_{i}$, as well as kinetic energy, $0.5 \rho u_{i}^{2}$, averaged over the volume of the flame brush and the cubic region of size $L$ located in the fuel immediately ahead of it. We were not able to find virtually any correlation between any of these quantities and either $S_{T}$ or $I_{0.15}$. As an example, Fig. 9 shows the representative correlation scatter plot of $I_{0.15}$ as a function of the normalized $x$-component of kinetic energy $E_{K, x}=\left(\rho u_{x}^{2}\right) /\left(\rho_{0} U_{l}^{2}\right)$ volume-averaged over the region discussed above. Similarly to the case of the distribution of $I_{0.15} \mathrm{vs} . A_{0.15} / L^{2}$ in Fig. 8, the increasing trend seen in the least squares fit is too weak to determine its statistical significance based on the available data.

These considerations suggest that the enhancement of $S_{n}$ over $S_{L}$ by turbulent transport does not provide a plausible explanation of the excess values of $S_{T}$ periodically developed by the flow. Consequently, a different mechanism must augment the effect of the increased $A_{T}$.

Instead of being the result of a uniform global increase of $S_{n}$ throughout the flame brush, accelerated burning can be caused by a significant increase in $S_{n}$ in isolated regions of the flame surface. In those regions, however, $S_{n}$ must be much larger than $S_{L}$ in order to significantly raise the overall burning speed of the turbulent flame.

Such local enhancement of $S_{n}$ cannot be caused by the local inhomogeneities in the thermodynamic state of the fuel 8 In particular, in a subsonic turbulent flow, such as the one considered here, with the turbulent Mach number $M a_{F}$ substantially less than unity (see Table 2), local variations of density or temperature are much too small to affect $S_{n}$ in any appreciable way. For instance, it requires an increase of fuel temperature of over $150 \mathrm{~K}$ to double $S_{n}$. We do not observe temperature fluctuations of such magnitude in the flow.

$S_{n}$ can also be increased by the substantially higher local turbulence intensity associated with intermittency. While we do observe intermittency in the flow field in our simulations, regions of large velocity enhancement are statistically too rare, their spatial extent is too small, and they are much too short lived to increase $S_{n}$ and, thus, to make any

\footnotetext{
${ }^{8}$ The increase of $S_{n}$ due to the global fuel heating by turbulence was taken into account in $S_{L}$, as discussed in $\S 2.2$
} 
significant contribution to the overall increase in $S_{T}$. Potential effects of turbulence intermittency on the flame have been studied by Pan et al. [37] in the context of the Rayleigh-Taylor-driven thermonuclear flames in the interior of a white dwarf during a Type Ia supernova explosion. Their results suggested the possibility that intermittency in the velocity field could locally disrupt and broaden the flame, which potentially would increase the local burning speed. It is not immediately clear, however, to what degree their conclusions apply to the system discussed here. The conditions in the white dwarf interior considered in [37] are substantially different from those in S1-S3. Moreover, their analysis did not consider any feedback of the flame on the turbulent field and, in particular, it did not include fluid expansion due to heat release which invariably affects intermittency of the flow. At the same time, the role of intermittency in the dynamics of turbulent flames does require further investigation. In particular, it would be important to extend the analysis of Pan et al. [37] by including the feedback of the flame on turbulence under conditions characteristic of chemical rather than thermonuclear flames.

Instead, we suggest that significant local increase of $S_{n}$ does indeed take place in the turbulent flame due to the flame collisions resulting in the creation of regions of large flame curvature. We discuss this process next.

\section{Accelerated burning caused by flame collisions}

\subsection{Local increase of $S_{n}$ in cusps}

According to the theory of flame stretch (see [7] for a review), when $L e=1$, the flame is not affected significantly [38] either by flow-induced strain or curvature. In particular, a stationary spherical flame supported by a point source of mass, which is an example of a curved unstrained flame, has an internal structure, and thus local burning velocity, identical to that of a planar laminar flame [7]. Such analysis, however, is typically carried out for a flame with the curvature radius $r_{c} \gg \delta_{L}$. Using the physical model and the Athena-RFX code described in $\S 2.1$, we also performed simulations of an idealized spherical and cylindrical flame propagating inward into the stationary fuel. The results of these simulations are, in fact, consistent with the theory. Furthermore, they show that $S_{n}=S_{L}$ not only when $r_{c} \gg \delta_{L}$, but up until the moment when the flame curvature becomes $\approx 1 / \delta_{L}$, i.e., until the flame effectively collapses onto itself. At this point, $S_{n}$ increases substantially. Beyond this moment, however, the flame itself effectively ceases to exist and, therefore, the local flame speed looses its meaning. Such an idealized situation is not representative of the conditions arising in an actual turbulent flame. Nonetheless, regions of large flame curvature $\sim 1 / \delta_{L}$ are frequently created in the high-speed turbulent flow considered here, and they naturally provide a mechanism for a significant local enhancement of $S_{n}$.

The results presented in $\S 3$ showed that intense turbulence causes tight folding and packing of the flame inside the flame brush. Consider the inverse of the surface density, which is a measure of an average separation between surface elements. Given that $\bar{\Sigma}_{0.15}=0.67 \mathrm{~mm}^{-1}$ in simulation $\mathrm{S} 3$, the average distance between individual flame sheets is $1 / \bar{\Sigma}_{0.15} \approx 1.49 \mathrm{~mm} \approx 4.7 \delta_{L}$. At the same time, $\Sigma_{0.15}$ can be as high as $0.97 \mathrm{~mm}^{-1}$ (Fig. $5 \mathrm{~h}$ ), resulting in an even lower separation of $\approx 3.2 \delta_{L}$. This is comparable to the full flame width $l_{F} \approx 2 \delta_{L}$ (cf. Fig. 7 in [11]). Such tightly folded flame configurations invariably result in frequent collisions of individual flame sheets.

Fig. 10 gives an example of such collision in S3. The figure shows the flame-brush structure at three times: $11.86 \tau_{e d}$ (upper panel), as well as $0.1 \tau_{e d}=3 \mu \mathrm{s}$ and $0.2 \tau_{e d}=6 \mu \mathrm{s}$ later (middle and lower panels, respectively). Initially, a region with a highly convolved flame develops in the flame brush (region A). At this time, $S_{n} \approx S_{L}$ locally throughout region A and changes in the flame configuration due to self-propagation can be neglected on timescales considered in Fig. 10. As turbulent motions continue to bring individual flame sheets closer to each other, the curvature radius of the flame becomes close to $\delta_{L}$, and the preheat zones begin to overlap substantially over an extended region of the flame surface. This marks the formation of two regions of high flame curvature $\sim 1 / \delta_{L}$ (regions B), which we will refer to as "cusps." Regions $\mathrm{C}$ in the lower panel show that $3 \mu \mathrm{s}$ later, the flame sheets have merged and formed two extended reaction zones, which suggests substantially accelerated burning in that area of the flame brush. Note also a rapid decrease of the area of the $Y=0.5$ isosurface represented with the thin black line. Comparison of regions $\mathrm{B}$ and $\mathrm{C}$ shows that this isosurface propagated over the distance $\approx 0.5 \mathrm{~mm}$ over the time $3 \mu \mathrm{s}$, which implies the propagation speed (but not the local burning speed) $\approx 55 S_{L}$. During the time shown in Fig. 10 , two other highly elongated regions of flame collision have formed near the upper face of the domain. Thus, Fig. 10 illustrates that flame collisions are ubiquitous in the turbulent flame, and they do produce regions of large flame curvature. Moreover, their evolution in Fig. 10 suggests substantial increase of both the local burning and propagation speeds in the cusps. 
Qualitatively, two different channels of cusp formation can be identified. They are shown schematically in Fig. 11 . In the first case, turbulent motions on a certain scale $\lambda$ with velocity $U_{\lambda}$ stretch and fold the flame. As a result, a narrow elongated structure is formed. When two flame sheets effectively collide, the curvature of the flame surface becomes $\sim 1 / \delta_{L}$ and the cusp is formed with a characteristic length $l_{c} \sim \lambda$. We will refer to this channel as "local flame collisions," since the cusp is formed by flame regions located close to each other both in 3D space and along the flame surface. In the second case, turbulent motions fold the flame on a larger scale, thus not increasing its curvature substantially. Two cusps are formed when two smoothly curved flame sheets collide. We will refer to this channel as "nonlocal flame collisions," since the colliding flame regions can be located far from each other along the flame surface and they can, therefore, originate from different regions of the flame brush. In this case, the characteristic length of the resulting cusp, $l_{c}$, can be significantly smaller than the scale, $\lambda$, of the motion that produced the cusp.

In an actual turbulent flame, cusps are often created under the combined action of these two channels, an example of which is seen in Fig. 10, Region A is initially formed by the flame surface being stretched and folded on a large scale, as in Fig. 11b. At the same time, smaller scale motions fold the flame surface locally, as in Fig. 11 b, forming smaller cusps seen in regions B.

While both local and nonlocal flame collisions are equivalent in terms of the resulting cusp properties, they differ in one important respect. In the first case, curvature of the flame surface at a specific point increases gradually until it becomes large marking the formation of a cusp. In the second case, the curvature remains small at all points of the flame surface until the actual moment of collision when two flame sheets merge and the region of large flame curvature is abruptly formed. These distinctive characteristics of the two processes will be important for the analysis of their relative efficiency to form cusps at a given turbulent intensity and system size.

\subsection{Structure and properties of cusps}

Formation of cusps (or "folds") by the wrinkled flame in a turbulent flow was first suggested by Karlovitz et al.[39] (also see discussion in [40]). Their dynamical role was first considered by Zel'dovich [41] (also see [42]), who suggested them as a mechanism of flame stabilization that prevents the unbounded exponential growth of the flame surface under the action of the Landau-Darrieus instability [43]. Such regions of large curvature would have a high propagation velocity, causing rapid annealing of the flame surface, and thus would provide an efficient mechanism to counterbalance flame instability [41]. It must be noted that both Karlovitz et al.[39] and Zel'dovich [41] assumed that cusps form due to the self-propagation of a wrinkled flame surface following the Huygens principle, rather than as a result of flame collisions by turbulence.

It was suggested early on both by Lewis and von Elbe [40] and Zel'dovich [41] that, due to the focusing of the heat flux into fuel, $S_{n}$ ceases to be equal to $S_{L}$ near the tip of the cusp, and the cusp region develops a structure similar to the tip of the Bunsen flame. The flame in [39, 40, 41, 42], however, was considered to be a gasdynamic discontinuity, and so its internal structure was ignored. As a result, the actual increase of the local flame speed in the cusp could not be determined.

In order to analyze quantitatively the effect of flame collisions and to determine the actual increase of $S_{n}$ in the resulting cusps, consider an idealized model of the cusps observed in Fig. 10, In particular, consider two symmetrically located planar flame sheets propagating with the speed $S_{L, 0}$ and approaching each other with the inclination angle to the mid-plane $\alpha$. At the point of collision, the flame sheets merge and form a cusp. Its properties, such as its structure, speed of propagation, and effective burning velocity, are determined in this configuration only by two parameters, $S_{L, 0}$ and $\alpha$. Such cusps can be formed through either of the channels shown in Fig. 11.

Fig. 12 illustrates the flame structure formed in this situation. It shows the distribution of the fuel mass fraction in two simulations performed for $\alpha=1^{\circ}$ and $\alpha=4^{\circ}$ with Athena-RFX using the same physical model as in simulations S1-S3. The domain has the resolution $\Delta x=\delta_{L} / 32$ and zero-order extrapolation boundary conditions on all sides. At $t=0$, two intersecting flame sheets were initialized with the exact structure of the planar laminar flame corresponding to the fuel temperature $T_{0}$ and density $\rho_{0}$ (see Table2). Uniform pressure $P_{0}$ and zero velocities were set in the domain at $t=0$. After the initial transient stage, the flame develops the structure shown in Fig. 12 The structure of the cusp itself does not change with time, provided that the planar flame sheets extend sufficiently far from it. Therefore, Fig. 12 can be viewed as a steady-state solution.

Fig. 12 shows that flame collision at very low inclination angles results in the formation of a highly elongated structure. It is formed by rapid heating and subsequent ignition of a larger amount of fuel than in the planar laminar 
flame due to the focusing of the thermal flux from two approaching flame sheets. Consequently, the extent of this structure is determined by the size of the region in which the preheat zones of approaching flames overlap and, thus, create the necessary enhanced thermal flux. Since the width of the preheat zone is $\approx \delta_{L, 0}$, very small values of $\alpha$ are required for the cusp to be substantially broader than $\delta_{L, 0}$. This can further be seen in the distributions of $Y, T$, and $\dot{Y}$ along the symmetry axis of the cusp shown in Fig. 13. The structure of the reaction zone in the cusp very quickly approaches that of the laminar flame with increasing $\alpha$, so that already at $\alpha \sim 4^{\circ}$ the two reaction zones become very similar. At the same time, the preheat zone of the cusp remains substantially broadened for much larger values of $\alpha$.

There are three characteristic speeds in this problem. Sufficiently far from the tip of the cusp, the flame locally propagates normal to its surface with the laminar flame speed $S_{L, 0}$, i.e., there $S_{n}=S_{L, 0}$. The cusp itself moves into the fuel with the phase velocity $U_{c}$, which results simply from the two inclined planar surfaces colliding with each other. This speed can be determined based on purely geometrical considerations [41],

$$
U_{c}=\frac{S_{L, 0}}{\sin \alpha}
$$

This cusp propagation speed, normalized by $S_{L, 0}$, is shown as a solid line in Fig. 14 along with $U_{c} / S_{L, 0}$ determined as the velocity of the leftmost point of the $Y=0.15$ isosurface in simulations for four values of $\alpha$. Eq. (23) is within $\lesssim 1 \%$ accuracy of the computed values. $U_{c}$ approaches the laminar flame speed as $\alpha \rightarrow 90^{\circ}$, i.e., when the two flame sheets cease to advance toward each other. At the opposite limit of small $\alpha, U_{c}$ can be quite large and, in principle, it could be infinite when $\alpha \rightarrow 0^{\circ}$. In particular, at $\alpha \approx 0.5^{\circ}, U_{c}$ becomes larger than the sound speed in cold fuel indicated with the horizontal dashed line in Fig. 14. Note also that at $\alpha=1^{\circ}$, the value of $U_{c} \approx 57 S_{L, 0}$ is comparable to the high propagation velocity $\approx 55 S_{L}$ of cusps in regions $\mathrm{B}-\mathrm{C}$ in Fig. 10 estimated in $\S 6.1$.

The third characteristic speed, which is the most important for us, is the local burning speed in the cusp, $S_{n}$. The broadened reaction zone at low flame-inclination angles (Figs. 12 and 13b) causes more fuel to be consumed per unit flame surface area than in the planar laminar flame, and, therefore, it leads to a larger local flame speed $S_{n}>S_{L, 0}$. Since the reaction-zone width is the largest at the tip of the cusp, $S_{n}$ has its maximum there and it gradually decreases to its laminar value $S_{L, 0}$ in the planar regions of the flame sufficiently far from the cusp. The maximum value of $S_{n}$ at the cusp tip can be found as

$$
S_{n}^{*} \equiv \max \left(S_{n}\right)=\frac{1}{\rho_{0}} \int \rho \dot{Y} d x=-\frac{B}{\rho_{0}} \int \rho^{2} Y \exp \left(-\frac{Q}{R T}\right) d x
$$

Here eq. (8) was used for $\dot{Y}$, and the integral is taken along the symmetry axis of the cusp shown in Fig. 12. Fig. 14b shows the computed values of $S_{n}^{*}$ normalized by $S_{L, 0}$ for the same four inclination angles as in Fig. 14a.

Two key conclusions emerge from Fig. 14 First, $S_{n}^{*}$ is substantially lower than $U_{c}$, being less by more than an order of magnitude at the values of $\alpha$ considered here. Second and most important, $S_{n}^{*}$ can indeed be much larger than $S_{L, 0}$ at small inclination angles.

\subsection{Connection between the increase of $S_{n}$ in cusps and $S_{T}$}

We are interested, however, not just in $S_{n}$ but, rather, in the burning speed of an extended flame region, as this is a direct equivalent of the turbulent flame speed. The total burning rate of the flame configuration shown in Fig. 12 is

$$
S_{c}=\frac{\dot{m}_{R}}{\rho_{0}}
$$

where $\dot{m}_{R}$ is the total mass of reactants converted into product per unit time. If the flame were to propagate everywhere with the speed $S_{L, 0}$, then $S_{c}=A_{Y} S_{L, 0}$, where $A_{Y}$ is the surface area of the flame in such a configuration. In this case all isosurfaces of $Y$ would be parallel to each other and, thus, $A_{Y}$ based on any value of $Y$ could be used. Otherwise,

$$
I_{Y}=\frac{S_{c}}{A_{Y} S_{L, 0}}>1
$$

Here $I_{Y}$ is completely equivalent to the definition used in eq. (16) in the context of the turbulent flame speed. Indeed, substituting the definition of $S_{T}$ given by eq. (11) into the definition of $I_{Y}$ given by eq. (16) shows that $\dot{m}_{R} / \rho_{0}=$ 
$I_{Y} A_{Y} S_{L, 0}=S_{c}$. Furthermore, as can be seen in Fig. 12, at low $\alpha$ isosurfaces of $Y$ do not have the same surface area. Therefore, similarly to the case of the turbulent flame, here $I_{Y}$ is also the function of $Y$. For consistency with previous results, we will consider $I_{0.15}$ based on the isosurface of $Y=0.15$ representing the peak reaction rate.

Since $S_{n}$ monotonically decreases away from the cusp, both $S_{c}$ and $I_{0.15}$ depend on the size of the flame region being considered. This size can be represented by the variable length $l_{c}$, illustrated in Fig. 12, which is defined as the distance from the leftmost point of the isosurface of $Y=0.15$ in the direction of cusp propagation. Considering different values of $l_{c}$ is equivalent to varying the fraction of the total flame surface area in Fig. 12. represented by the planar section in which $S_{n} \approx S_{L, 0}$. Consequently, this allows one to vary the relative contribution to $S_{c}$, and thus to $I_{0.15}$, of the cusp in which $S_{n}>S_{L, 0}$.

Fig. 15 shows $I_{0.15}$, calculated using eqs. 25)-(26), as a function of $l_{c} / \delta_{L, 0}$ for four values of $\alpha$. At smaller $l_{c}$ close to the width of the reaction zone for a given $\alpha$ (see Fig. 13b), $I_{0.15}$ essentially reflects only the values of $S_{n}$ in the immediate vicinity of the tip of the cusp, where $S_{n}$ is close to $S_{n}^{*}$. On the other hand, at larger $l_{c}$, the surface area of the planar flame region, in which $S_{n} \approx S_{L, 0}$, is larger, and so this region represents a greater fraction of the total surface area of the flame configuration shown in Fig. 12. As a result, the contribution of the planar flame starts to dominate that of the cusp, which causes $I_{0.15} \rightarrow 1$ as seen in Fig. 15. Note that at lower $\alpha$, the region in which $S_{n}>S_{L, 0}$ extends progressively further from the cusp tip. In particular, at $\alpha=1^{\circ}, I_{0.15}$ is substantially greater than unity even at $l_{c}>>\delta_{L, 0}$. At the same time, already at $\alpha=4^{\circ}, I_{0.15}$ drops to $\lesssim 1.04$ at $l_{c} \approx 5 \delta_{L, 0}$ which shows that $S_{n}$ recovers its laminar value within a few laminar flame widths from the cusp tip.

These results show that the formation of a cusp due to the collision of planar flame sheets produces values of $I_{0.15}$ substantially larger than unity and comparable to those observed in the simulations presented here. As a result of a higher local flame speed in a cusp, its contribution to the global turbulent burning speed is disproportionately large compared to the fraction of the instantaneous flame surface area in it. A more complex flame configuration, consisting of multiple planar flame sheets that approach each other and collide forming multiple cusps, can then be considered. In this system, for instance, if the flame surface density is increased, flame sheets will get closer to each other causing flame collisions to become more frequent and the resulting cusps to become more numerous. Consequently, the fraction of the flame surface area contained in cusps will increase and $I_{0.15}$ will grow, which is demonstrated by the increase in $I_{0.15}$ with decreasing $l_{c}$.

This simplified model demonstrates the mechanism through which $S_{T}$ can produce an exaggerated response to the increase in $A_{T}$. Yet it does not take into account the full complexity of an actual turbulent flame. Such flame does not consist of perfectly planar flame sheets that merge, but instead it is constantly wrinkled and folded on a variety of scales (Fig. 11). As a result, cusps will form in a multitude of configurations. For instance, in addition to the purely $2 \mathrm{D}$ situation considered here, there will also exist 3D flame collisions which will result in even higher local flame speeds in the cusps and, thus, will have an even larger effect on the magnitude of $I_{0.15}$. The probability of the formation of such more complex flame collisions, however, will rapidly decrease with the increase in their complexity [3, 23]. Nevertheless, in order to predict a particular value of $I_{0.15}$ which can be expected in the turbulent flow of a specific intensity, it is necessary to understand the types of all cusps which can form, the local flame speed of each type, its probability of formation, and, thus, the contribution of each type to the exaggerated response of $S_{T}$. Such detailed analysis is the subject for future studies.

\subsection{Effect of cusps on the diffusion velocities in the turbulent flame}

Changes in the local flame structure (Figs. 12 and 13) in cusps will result in modified local diffusion velocities, $D|\nabla Y|$. This is the direct consequence of focusing of the diffusive fluxes which alters the molecular and thermal transport in regions of high flame curvature. It is, therefore, instructive to compare the distribution of the diffusion velocities in the idealized cusps considered above and in the actual turbulent flame.

We calculated the surface-averaged normalized diffusion velocity,

$$
\frac{V_{D}(Y)}{S_{L}}=\frac{\int_{A_{Y}} D|\nabla Y| d A}{A_{Y} S_{L}},
$$

for a set of discrete values of $Y$ in simulation S3 and in the cusp formed at the low inclination angle $\alpha=1^{\circ}$ shown in the upper panel of Fig. 12. Here the diffusion coefficient $D$ is given by eq. (7) and the integration is performed over an isosurface of a given $Y$. In $\mathrm{S} 3$, the values of $V_{D}(Y)$ change with time due to the fuel heating by turbulence. In order 
to take this effect into account, we normalized the instantaneous $V_{D}(Y)$ by the corresponding actual instantaneous laminar flame speed $S_{L}$, as was discussed in $\S 2.2$ The resulting distributions were subsequently time-averaged. In the idealized cusp calculation, $S_{L}=S_{L, 0}$ at all times, and the instantaneous flame structure shown in Fig. 12. represents a steady state. The obtained $\overline{V_{D}(Y) / S_{L}}$ for both calculations is shown in Fig. 16. We also show two distributions of $V_{D}(Y)$ representing the exact planar laminar flame solutions constructed for two fuel temperatures, namely $T_{0}=293$ $\mathrm{K}$ and $320 \mathrm{~K}$, which correspond to the average fuel temperatures at the beginning and at the end of S3. These were also normalized by the corresponding $S_{L}$.

Fig. 16 shows that the flame configuration in Fig.12 (upper panel), which consists both of a cusp and the extended planar flame regions, has diffusion velocities in the reaction zone $\lesssim 10 \%$ lower than in the planar laminar flame. At the same time, a similar decrease of $V_{D} / S_{L}$ in a laminar flame is produced as a result of fuel heating to $320 \mathrm{~K}$. On the other hand, the time-averaged distribution of $\overline{V_{D} / S_{L}}$ inside the turbulent flame is also similarly lower in comparison with the base laminar case at fuel temperature $T_{0}=293 \mathrm{~K}$. This fact, along with the $1 \sigma$ standard deviation of the instantaneous values of $V_{D} / S_{L}$ shown as the shaded gray region, indicates that the observed decrease of the diffusion velocities in $\mathrm{S} 3$ is consistent with the combined effect of fuel heating and the formation of cusps.

\subsection{Criterion for onset of the regime of flame evolution influenced by cusps}

Properties of cusps discussed above allow us to determine when the evolution of a turbulent flame can be expected to become affected by cusp formation, thereby, leading to values of $I_{0.15}$ substantially larger than unity. The results presented in this paper show that such high $I_{0.15}$ require a large number of cusps to exist or, in other words, the flame must have large curvature $\sim 1 / \delta_{L}$ over a significant fraction of its total surface. This means that the flame must be tightly folded with a characteristic separation $1 / \Sigma_{T}$ comparable to the full flame width $l_{F}$, which is indeed the situation observed in the simulations discussed here.

Consider now the two channels of cusp formation discussed in $\$ 6.1$ and illustrated in Fig. 11. Nonlocal flame collisions cannot create a tightly packed turbulent flame since, by definition, they fold the flame only on large scales causing flame sheets to move toward each other. Large curvature forms only at the moments of collision after which the large speed of cusp propagation, $U_{c}$, causes rapid annealing of the flame surface. Consequently, a tightly packed flame can form only if local flame collisions are an efficient process capable of folding the flame on scales $\sim \delta_{L}$ not only in isolated regions but consistently throughout the flame surface.

It was discussed in $\S 6.2$ (Fig. 14) that $U_{c}$, unlike $S_{n}^{*}$, is much greater than $S_{L}$ even at large flame inclination angles, and, thus, it increases gradually with curvature. Therefore, $U_{c}$ is the primary factor responsible for smoothing the flame surface while it is being wrinkled by turbulence. In particular, as the turbulent motions $U_{\lambda}$ shown in Fig. 11 a fold the flame thereby progressively increasing its curvature, they must overcome the straightening action of the growing $U_{c}$. The formation of a cusp, as well as its structure, are then determined by the balance between $U_{\lambda}$ and $U_{c}$.

Next consider a section of an idealized curved flame front, schematically shown in Fig. 17, containing a cusp formed through a local flame collision (Fig. 11 ). The flame is perturbed with a wavelength $\lambda_{c}$ and an amplitude $l_{c}$. This type of structure was considered by Zel'dovich [41] as a model of a flame formed under the action of the LandauDarrieus instability in order to analyze the stabilizing effect of cusps. The same structure, however, can emerge under the action of any destabilizing process, e.g., the Rayleigh-Taylor instability or, in our case, turbulence.

In this case, the rate of decrease of the cusp amplitude is [41]

$$
\left(\frac{d l_{c}}{d t}\right)_{-}=-S_{L}\left(\frac{1}{\sin \alpha}+1\right)
$$

The angle $\alpha$ and, thus, $\left(d l_{c} / d t\right)_{-}$, will change with the cusp amplitude. In order to relate $\alpha$ and $l_{c}$, a parabolic shape of the flame was assumed in [41], and eq. (28) can then be rewritten as

$$
\left(\frac{d l_{c}}{d t}\right)_{-}=-8 S_{L} \frac{l_{c}^{2}}{\lambda_{c}^{2}} .
$$

The net rate of change of $l_{c}$ is determined by the balance of the stabilizing process described by eq. (29) and a destabilizing process, namely

$$
\frac{d l_{c}}{d t}=\Psi-8 S_{L} \frac{l_{c}^{2}}{\lambda_{c}^{2}}
$$


In the turbulent flow, flame perturbations can be assumed to grow linearly in time as they are being stretched by the turbulent speed $U_{\lambda_{c}}$ characteristic of the scale $\lambda_{c}$. This gives the growth rate $\Psi=U_{\lambda_{c}}$. By setting $d l_{c} / d t=0$, the limiting value of $l_{c}\left(\lambda_{c}\right)$ can be determined. It was shown by Khokhlov [23] in the context of Rayleigh-Taylor-unstable flames that nonlinear flame stabilization due to cusp propagation can be expected to fail once $l_{c} \gtrsim \lambda_{c}$. Therefore, by setting $l_{c}=\lambda_{c}, \Psi=U_{\lambda_{c}}$, and $d l_{c} / d t=0$ in eq. (30), the critical value of the turbulent velocity on a given scale $\lambda_{c}$ can be found [23]

$$
U_{\lambda_{c}}=8 S_{L}
$$

This is the value of $U_{\lambda_{c}}$ that is needed to overcome the stabilizing effect of cusps and, thereby, to deform the flame on scale $\lambda_{c}$. This result was used to demonstrate that, in order for the flame to be unstable at a wavelength $\lambda_{c}$ under the action of turbulent motions, the turbulent speed on that scale must be substantially larger than $S_{L}[23]$.

This shows that given a specific turbulent cascade, the flame will be stabilized on scales smaller than some critical wavelength $\lambda_{c}^{\text {crit }}$ on which the condition in eq. (31) is satisfied. In order for the cusps to have a pronounced effect on the flame evolution, the flame must be folded on scales comparable to the full flame width $l_{F}$. Therefore, by setting $\lambda_{c}^{c r i t}=l_{F}$ and using eq. 31, we find that the flame will be curved on scales $\sim l_{F}$ when turbulent velocity on that scale becomes $\sim 8 S_{L}$. In a turbulent flow with the Kolmogorov energy spectrum, the corresponding critical value of the integral turbulent velocity then is

$$
U_{l}^{c r i t} \approx 8 S_{L}\left(\frac{l}{l_{F}}\right)^{1 / 3}
$$

This gives the critical values of the Karlovitz number, $K a^{\text {crit }}$, and the Damköhler number, $D a^{\text {crit }}$, written using their traditional definitions [5]

$$
\begin{aligned}
& K a^{c r i t}=\frac{\tau_{F}}{\tau_{\eta}}=\left(\frac{l_{F}}{L_{G}}\right)^{1 / 2}=8^{3 / 2} \approx 20, \\
& D a^{c r i t}=\frac{\tau_{T}}{\tau_{F}}=\frac{l S_{L}}{l_{F} U_{l}}=\frac{1}{8}\left(\frac{l}{l_{F}}\right)^{2 / 3} .
\end{aligned}
$$

Here $\tau_{F}=l_{F} / S_{L}$ is the characteristic flame time, $\tau_{\eta}$ is the Kolmogorov time, $\tau_{T}=l / U_{l}$ is the characteristic turbulent time on the integral scale, and $L_{G}=l\left(S_{L} / U_{l}\right)^{3}$ is the Gibson scale.

Fig. 18 presents a traditional combustion regime diagram [5]. The orange region shows the range of the regimes, in which $I_{0.15}$ is expected to be larger than unity, according to eq. (33). The solid red square corresponds to the simulations discussed here. We also determined the time-averaged value of $I_{0.15}$ in a simulation similar to S2, but with approximately half the turbulent intensity which, however, was still above the $K a^{\text {crit }} \approx 20$ line. In this calculation, represented with the open red square in Fig. 18 , we found $\bar{I}_{0.15} \approx 1.1$, compared with $\bar{I}_{0.15} \approx 1.14$ found in simulation S3 (Table 4). Details of this calculation will be presented in a separate paper.

Despite the simplicity of the cusp model used here, eqs. (32)-(33) provide a rather accurate criterion for the onset of the regime of the turbulent flame evolution in which the contribution of cusps is expected to become important. In particular, the decrease in the value of $\bar{I}_{0.15}$ between the two turbulent intensities considered in Fig. 18 suggests that below the $K a^{\text {crit }} \approx 20$ line $I_{0.15}$, on average, deviates from unity at most by a few percent.

\subsection{Effect of different turbulent intensities and system sizes}

For the given reaction-diffusion model and fuel properties, the only two free parameters in the system considered here are the turbulent intensity and system size represented by the turbulent integral velocity, $U_{l}$, and scale, $l$. Consequently, the question arises, how will the distributions of $S_{T} / S_{L}$ and, more importantly, of $I_{0.15}$ as a function of $A_{0.15} / L^{2}$ shown in Figs. $6 \mathrm{~b}$ and Fig. 8 vary with the change in $l$ and $U_{l}$ ?

The results presented here and in [11] demonstrated the following key property of the flame interaction with highspeed turbulence. Turbulent motions are much more efficient at folding the flame with increasingly greater curvature than at disrupting its internal structure. This creates tightly packed turbulent flames which, in regions of low curvature, retain locally the laminar structure and velocity. Therefore, an increase in $U_{l}$ creates both larger flame surface area, $A_{T}$, and density, $\Sigma_{T}$.

On the other hand, $l$ does not affect $\Sigma_{T}$. The analysis in $\S 6.5$ showed that flame folding on a given scale is controlled only by the turbulent velocity on that scale rather than by the total range of scales present in the system. Instead, a larger system size results in a wider turbulent flame and, thus, in larger values of $A_{T}$. This follows from the 
fact that, in the high-speed regime, the turbulent flame width, $\delta_{T}$, is primarily determined by $l$ (and, therefore, by the driving scale, $L$ ) rather than by $U_{l}$, as was discussed in [11] based on the comparison of the results of that work with the results of Aspden et al. [9]. A similar conclusion was also reached by Peters [5] (cf. eq. (2.175) therein).

At the same time, a given $U_{l}$ and $l$ is not characterized by a single $S_{T}, A_{T}$, and $\Sigma_{T}$. Instead, a variety of different flame configurations are realized in the course of flame evolution, as was seen above (cf. Fig.6p). These configurations have different $A_{T}$, which is the primary factor determining the distribution of $S_{T}$. More importantly, however, at a given $A_{T}$, there are also different numbers and types of cusps present in the flame. This creates a scatter in the distribution of $S_{T} / S_{L}$ vs. $A_{T} / L^{2}$, which in the absence of cusps would lie on the $S_{T} / S_{L}=A_{T} / L^{2}$ line. These cusps are the result of the combined action of the two cusp-formation channels discussed in $\$ 6.1$. Consequently, this scatter reflects the dependence of the two types of flame collisions on $A_{T}$ and $\Sigma_{T}$.

The efficiency of local flame collisions (Fig. 11 $)$ is independent of either $A_{T}$ or $\Sigma_{T}$, since this process of cusp formation is controlled only by the intensity of turbulent motions which stretch and fold the flame at a certain point of its surface. As a result, the probability of cusp formation per unit flame surface area in this process does not increase with $A_{T}$ or $\Sigma_{T}$. Consequently, if only local flame collisions were present in the system, than the fraction of $A_{T}$ contained in cusps would grow proportionally to the increase in $A_{T}$, and the resulting $I_{0.15}$ would not change.

On the other hand, the efficiency of nonlocal flame collisions (Fig. 11 b) does depend not only on $U_{l}$, but also on $A_{T}$ and $\Sigma_{T}$. To show this, the following qualitative model can be used (see also [23]). In this process, cusps are created due to collisions of flame sheets with very low curvature. Therefore, consider an idealized structure consisting of planar flame sheets discussed in $\$ 6.3$ If the flame surface density of this configuration is $\Sigma_{T}$, then the average flame separation is $1 / \Sigma_{T}$. Flame sheets will move toward each other with the speed $S_{L}+U_{T}$, where $U_{T}$ is some characteristic turbulent velocity responsible for the advective transport of the flame. Since $U_{l}$ is the largest velocity of coherent turbulent motions, then $U_{T}$ can be approximated with $U_{l}$. Consequently, the flame sheets will merge and annihilate in the time $t_{c} \sim 1 /\left(\Sigma_{T}\left(S_{L}+U_{l}\right)\right)$. The total number of collisions and, thus, the total number of resulting cusps, $N_{c}$, will depend on the total surface area of the flame sheets times the frequency of their collisions, i.e., $N_{c} \propto A_{T} / t_{c} \propto A_{T} \Sigma_{T}\left(S_{L}+U_{l}\right)$. Since $A_{T}$ and $\Sigma_{T}$ are well correlated ( $\left.\$ 3.3\right), A_{T}$ can be viewed as simply proportional to $\Sigma_{T}$, which gives $N_{c} \propto\left(S_{L}+U_{l}\right) A_{T}^{2} \propto\left(S_{L}+U_{l}\right) \Sigma_{T}^{2}$. Therefore, the number of cusps formed per unit flame surface area will be

$$
\frac{N_{c}}{A_{T}} \propto\left(S_{L}+U_{l}\right) A_{T} \propto\left(S_{L}+U_{l}\right) \Sigma_{T}
$$

These considerations show that at a given $l$ and $U_{l}$, the distribution of values of $I_{0.15}$ has two contributions: one due to local flame collisions independent of $A_{T}$ and $\Sigma_{T}$, and the other due to nonlocal flame collisions, which increases with $A_{T}$ and $\Sigma_{T}$. In smaller systems and at lower turbulent intensities, or, equivalently, at lower $A_{T}$ and $\Sigma_{T}$, the first process is dominant. This can be seen in the distribution of $I_{0.15}$ vs. $A_{0.15} / L^{2}$ in Fig. 8, which shows almost no dependence on $A_{T}$. Note, however, a weak increasing trend suggested by the least squares fit. In particular, the lowest values of $I_{0.15}$ become progressively larger with increasing $A_{0.15}$ approaching $\approx 1.1$ as $A_{0.15} / L^{2} \rightarrow 5$. While the statistical significance of this trend must be confirmed through further studies, this trend would be indicative of the contribution of nonlocal collisions. The analysis given above suggests that such dependence of $I_{0.15}$ on $A_{T}$ will become pronounced in larger systems or at larger turbulent intensities.

The fact that $I_{0.15}$ must depend on $A_{T}$ also follows from a different argument. Consider first a limiting value of $\Sigma_{T}$. The fact that the flame is not an infinitely thin surface means that such a limit does exist. Once $\Sigma_{T}$ becomes large enough so that all of the flame surface comes into contact with itself, $\Sigma_{T}$ cannot increase further. This maximum value $\Sigma_{T}^{\max }$ can be estimated by assuming that the minimum flame separation is equal to the full flame width $l_{F}$. Then 10

$$
\Sigma_{T}^{\max } \equiv \Sigma_{0.15}^{\max }=\frac{1}{l_{F}}
$$

\footnotetext{
${ }^{9}$ This expression is very similar to the destruction term often used in the balance equation of the flame surface density (see [44] for a review of a number of such models). This is also analogous to the rate of collisions of gas molecules, with $\Sigma_{T}$ playing the role of number density and the turbulent intensity playing the role of temperature in determining the speed with which constituents approach each other.

${ }^{10}$ Note that $\Sigma_{T}^{\max }$ is different from $\Sigma_{\max }$ typically used in combustion research and discussed in $\S 3.2$
} 
Using the definition of $\Sigma_{Y}$ given by eq. (14), the corresponding limiting value of the flame surface area then is

$$
\frac{A_{T}^{\max }}{L^{2}} \equiv \frac{A_{0.15}^{\max }}{L^{2}}=\Sigma_{T}^{\max } \delta_{T}=C_{1} \frac{L}{l_{F}}=\left(C_{1} C_{2}\right) \frac{l}{l_{F}} .
$$

Here, $C_{1}=\delta_{T} / L$ and it typically is $\approx 2-4$ based on the results of [11, 9]. The $C_{2}=L / l$ and in the case of the Kolmogorov turbulence $C_{2} \approx 4$. In our case $l_{F} \approx 2 \delta_{L, 0}$ (cf. Fig. 7 in [11]). Therefore, based on the values of $L$ and $l$ given in Table 3 for the system considered here, $\Sigma_{T}^{\max } \approx 1.5 \mathrm{~mm}^{-1}$ and $A_{T}^{\max } / L^{2} \approx 7-8$.

The situation when all of the flame surface is in contact with itself corresponds to the infinite local flame speed and, thus, infinite $S_{T}$. This is equivalent to the complete domination of the nonlocal process of cusp formation when the flame sheets collide simply because they are very close to each other and not as a result of the folding action of turbulence. Therefore, as $\bar{A}_{T} / L^{2} \rightarrow A_{T}^{\max } / L^{2}$, both $\overline{S_{T} / S_{L}} \rightarrow \infty$ and $\bar{I}_{0.15} \rightarrow \infty$. On the other hand, as $\bar{A}_{T} / L^{2} \rightarrow 1$, $\overline{S_{T} / S_{L}} \rightarrow 1$ and, thus, $\bar{I}_{0.15} \rightarrow 1$. This shows that $\bar{I}_{0.15}$ cannot be constant. Instead, it has to be a monotonically increasing function of $A_{T}$, as suggested.

In summary, the following picture emerges. At a given $l$, lower turbulent intensities cause the flame to be less convolved and, thus, to develop less surface area. As a result, the overall distribution of $S_{T} / S_{L}$ vs. $A_{T} / L^{2}$ would shift toward smaller values of $A_{T} / L^{2}$ and, thus, $S_{T} / S_{L}$. Furthermore, the efficiency of both local and nonlocal flame collisions would decrease. As a result, cusps would become infrequent representing only a small fraction of the total flame surface. Consequently, the flame almost everywhere would propagate locally with its laminar speed, thus causing the scatter in the distribution of $S_{T} / S_{L}$ to decrease and values of $S_{T} / S_{L}$ to collapse onto the $S_{T} / S_{L}=A_{T} / L^{2}$ line in the limit of $U_{l} \rightarrow 0$. Note, however, that while at $U_{l}<U_{l}^{\text {crit }}$ given in eq. (31) local flame collisions practically cease to occur, individual cusps can still form through nonlocal collisions. Since these depend on $A_{T}, I_{0.15}$ would approach unity with decreasing $U_{l}$ more slowly in larger systems that have greater $A_{T}$ than in smaller systems.

At larger $U_{l}$, the behavior would be opposite. Not only would the overall distribution of $S_{T} / S_{L}$ vs. $A_{T} / L^{2}$ shift to larger values of $A_{T} / L^{2}$ and $S_{T} / S_{L}$, but also the efficiency of both local and nonlocal flame collisions would increase, causing a much larger scatter of the distribution. Furthermore, at high turbulent intensities, flame configurations with very few cusps and, thus, $I_{0.15} \approx 1$, would become increasingly less likely to occur. Therefore, larger $U_{l}$ will cause not only a higher rate of burning due to the increase in $A_{T}$, but it will also periodically lead to a substantially more exaggerated response of $S_{T}$ to such increase in $A_{T}$. Finally, dependence of $I_{0.15}$ on $A_{T}$ would be more pronounced due to the increased contribution of nonlocal collisions and, at a given $U_{l}$, larger systems would tend to have higher values of $I_{0.15}$.

\section{Conclusions}

This work continued the analysis of the set of three numerical simulations first presented in [11]. These calculations model the interaction of a premixed flame with high-speed, subsonic, homogeneous, isotropic turbulence in an unconfined system, i.e., in the absence of walls and boundaries. The turbulent r.m.s. velocity, $U_{r m s}$, is $\approx 35$ times larger than the laminar flame speed, $S_{L}$. The resulting Damköhler number based on the turbulent integral scale and velocity is $D a=0.05$.

It was demonstrated in [11] that this system represents turbulent combustion in the thin reaction zone regime. Even in the presence of such intense turbulence, the flame brush consists of the highly convolved flame with its reactionzone structure virtually identical to that of the planar laminar flame and with the preheat zone broadened by a factor $\approx 2$. The fact that turbulence is unable to penetrate and disrupt the internal structure of the reaction zone suggested that the flame must be propagating locally with the speed $S_{L}[11]$. This raised the following question: is the turbulent flame speed, $S_{T}$, in the thin reaction zone regime completely determined by the increase in the flame surface area, $A_{T}$, as it is the case in the wrinkled and corrugated flamelet regimes? Here we summarize the main findings of our study of this issue.

Analysis of the area and density, $A_{Y}$ and $\Sigma_{Y}$, of the fuel mass-fraction isosurfaces showed that the flame, folded inside the flame brush in the presence of high-speed turbulence, cannot be viewed as a thin uniform structure in which all isosurfaces are parallel to each other. Different regions of the flame have quite different response to the action of turbulence. In the higher-resolution calculations $\mathrm{S} 2$ and S3, both $A_{Y}$ and $\Sigma_{Y}$, on average, increase monotonically through the flame with increasing $Y$, which leads to the distinctive inverted-S shape of the time-averaged distributions 
of $A_{Y}$ and $\Sigma_{Y}$. Consequently, isosurfaces of higher $Y$ are folded by turbulence on progressively smaller scales. This causes substantially finer wrinkling of the flame surface on the fuel side than on the product side, as observed in [11]. Furthermore, in the reaction zone, $A_{Y}$ grows due to both much tighter folding of isosurfaces and the increase in the overall width of the flame brush, with the first process being more dominant. On the other hand, in the preheat zone, isosurfaces of $Y$ are packed primarily on small scales without contributing to the increase of $\delta_{T}$.

These properties of the distributions of $\bar{A}_{Y}$ and $\bar{\Sigma}_{Y}$ showed that, in the thin reaction zone regime, the definition of the flame surface area must be revisited before the relation between $A_{T}$ and $S_{T}$ can be considered. In particular, it must be determined which value of $Y$ fully and accurately characterizes the evolution and global properties of the turbulent flame. To answer this question, we analyzed the correlation of $A_{Y}$ with quantities that characterize both the energetics and the global structure of the turbulent flame, namely its speed, $S_{T}$, and width, $\delta_{T}$. This analysis demonstrated that global properties of the turbulent flame in the thin reaction zone regime are accurately represented by the structure of the region of peak reaction rate. For the reaction-diffusion model used in this work, this corresponds to $Y \approx 0.15$. Therefore, the isosurface of this value of $Y$ must be viewed as the flame surface and $A_{T}=A_{0.15}$. Correspondingly, the flame surface density is $\Sigma_{T}=\Sigma_{0.15}$.

Large values of $\Sigma_{T}$ developed in the course of the system evolution, on one hand, and the absence of any broadening of the reaction zone observed in [11], on the other, demonstrated the following important fact. High-intensity turbulence is much more efficient at tightly packing the flame inside the flame brush than at disrupting and broadening its internal structure (also see [5]).

The distribution of $S_{T} / S_{L}$ as a function of $A_{T} / L^{2}$ and, more specifically, the ratio $I_{0.15}=\left(S_{T} / S_{L}\right) /\left(A_{T} / L^{2}\right)$ showed that, in the thin reaction zone regime, the magnitude of $S_{T}$ is controlled primarily by the increase of the flame surface area. In particular, on average $I_{0.15} \approx 1.14$. As a result, a variety of different flame configurations realized over time, with both $A_{T}$ and $\Sigma_{T}$ varying by over a factor of $\approx 3$, cause $S_{T} / S_{L}$ also to vary over a broad range of values $\approx 1.6-5.5$. Such significant changes in the burning rate demonstrate that, in order to properly characterize the turbulent flame evolution in this regime, it is not sufficient to consider only the time-averaged $\bar{S}_{T}$. Instead, the full PDF of the instantaneous values of $S_{T}$ must be determined.

At the same time, the distribution of $S_{T} / S_{L}$ exhibited another important characteristic. In the course of the system evolution, $S_{T} / S_{L}$ varies from within only a few percent of $A_{T} / L^{2}$ to as high as $30 \%$ larger than $A_{T} / L^{2}$. This led to the following key conclusion of this work. In the thin reaction zone regime given a sufficiently high turbulent intensity, $S_{T}$ periodically exhibits a substantially exaggerated response to the increase in $A_{T}$. Such accelerated burning in the turbulent flame means that an additional mechanism must augment the effect of the increase of the flame surface area.

Our analysis showed that tightly packed flame configurations, produced by high-speed turbulence, have the average flame separation of only a few full flame widths. This results in frequent flame collisions that lead to the formation of regions of high flame curvature $\gtrsim 1 / \delta_{L}$, or "cusps." The resulting significant focusing of the thermal flux over an extended region of the flame surface can significantly increase the local burning speed, $S_{n}$, in the cusp over its laminar value, $S_{L}$. These large values of $S_{n}$ cause the contribution of cusps to the total $S_{T}$ to be disproportionately large compared to the flame surface area they contain. This provides a natural mechanism to accelerate burning in the turbulent flame above what can be attributed to the increase in $A_{T}$, even in reactive mixtures characterized by $L e=1$. The increase of $S_{n}$ in cusps is inherently local, and it does not require the flame to be broadened and its speed increased by small-scale turbulent transport, in agreement with the results of [11].

Therefore, our results indicate that cusp formation is a crucial process in the turbulent flame evolution responsible for controlling $S_{T}$. It is capable of accelerating burning in addition to the two processes originally suggested by Damköhler [1], namely the increase of $A_{T}$ and the enhancement of the local flame speed by turbulence. Furthermore, both the criteria given by eqs. (31)-33) and the results of numerical simulations show that effects of cusps become pronounced well within the thin reaction zone regime and, in particular, at $K a^{\text {crit }} \gtrsim 20$ (Fig. 18). Above this critical value of $K a$, increase of $S_{T}$ over $S_{L}$, on average, exceeds by $\gtrsim 10 \%$ the corresponding increase of $A_{T}$. This shows that a substantially accelerated turbulent burning can arise due to cusps at much lower turbulent intensities than would be necessary to disrupt the flame and increase its local speed by small-scale turbulence.

Above $\mathrm{Ka}^{\text {crit }}$ (Fig. 18), the flame evolution likely remains substantially affected by cusp formation at all turbulent intensities, as long as there is folding of the flame by large-scale motions. This is the result of the fact that turbulence is more efficient at packing the flame than at broadening it. In particular, at high enough values of $U_{l}$, turbulence will eventually break the internal flame structure, which will increase $S_{n}$. This will, in turn, increase the right-hand side of eq. (31), thereby, enhancing the stabilizing effect of cusps. On the other hand, the flame width will also grow, 
increasing the critical flame separation necessary for the onset of the regime influenced by cusp formation. Since $S_{n}$ is determined by small-scale turbulence while flame folding is governed by the faster large-scale motions, it is unlikely that $S_{n}$ can grow fast enough to compensate for the increase in both $l_{F}$ and the turbulent speeds which fold the flame. Consequently, at higher $U_{l}$, i.e., even in the broken reaction zone regime, cusps most likely continue to provide a significant enhancement of $S_{T}$. This issue, however, requires further investigation in future studies.

The effect of flame collisions on the turbulent flame speed suggests that, even at high turbulent intensities, $S_{T}$ does not become independent of the laminar flame speed. Analysis given in $\$ 6.5$ and, in particular, eqs. (31) - (32), showed that flame folding by turbulence, and thus the efficiency of cusp formation, is controlled by $S_{L}$. For instance, at the same $U_{l}$, the reactive mixture with higher $S_{L}$ will develop a less convolved flame with fewer cusps than the one with lower $S_{L}$. Consequently, the exaggerated response of $S_{T}$ to the increase of $A_{T}$ due to cusps will vary with $S_{L}$.

The results presented in this work show that for a large range of turbulent intensities and system sizes, knowledge of $A_{T}$ is not sufficient to predict the magnitude of $S_{T}$, even if the reaction-zone structure remains unaffected by turbulence. For instance, in the regime considered here, using $A_{T}$ as a guide would result in errors as high as $30 \%$. Such errors are quite substantial, given that the flow evolution is typically very sensitive to the rate of energy release. Therefore, it is particularly important to account for the effects of cusps in subgrid-scale models that primarily focus on determining the evolution of $A_{T}$ (e.g., [23]). Furthermore, when constructing such modified subgrid-scale models, flame propagation can no longer be viewed as a local process. In particular, $S_{n}$ at each point of the flame surface is no longer determined only by the local thermodynamic state of the flow or by turbulent motions on scales $\lambda<\delta_{L}$. Instead, in the presence of cusps, $S_{n}$ is also controlled by long-range velocity correlations, which produce flame collisions and can span the full size of the system.

The formation of cusps and the resulting rapid flame propagation in certain regions are a crucial part of the turbulent flame-brush evolution in the high-speed regime. Thus, properly capturing this regime in numerical models requires the domain size to be larger than the integral scale in order to accommodate the folding of the flame by turbulence. Making the domain smaller than $l$ would significantly hamper this process, while making the domain smaller than $\sim \delta_{L}$ would completely eliminate it.

The increase of $S_{n}$ in cusps is discussed here for $L e=1$. It is well known, however, that when $L e \neq 1, S_{n}$ varies with the flame curvature, even when it is $\ll 1 / \delta_{L}$ [3]. Therefore, due to the large curvature in cusps, any imbalance between thermal and diffusion fluxes can significantly exacerbate or suppress the enhancement of $S_{n}$, depending on the value of $L e$. Therefore, the role and properties of cusps in non-equidiffusive reactive mixtures must be investigated in future studies. Furthermore, it is important to extend the results presented in this work by considering the effects of detailed chemistry, in particular, to address the role of flame quenching and re-ignition at such high turbulent intensities that are necessary for efficient cusp formation.

Finally, two possibilities exist for the flame evolution in the thin reaction zone regime. If the turbulent intensity is not too high, an equilibrium is established between flame-surface creation by turbulence and its rapid destruction in cusps. This results in the turbulent flame propagating in a steady state, which is the situation observed here. On the other hand, if the turbulent intensity continues to rise, eventually $\Sigma_{T}$ and $A_{T}$ can approach their limiting values given by eqs. (35) - (36). In this case, both $S_{T} / S_{L}$ and $S_{T} / A_{T}$ can become arbitrarily large, as was discussed in $\S 6.6$. Such singular behavior suggests that in reality, unless turbulence substantially alters the flame properties, the steady state must cease to exist and the system must undergo a qualitative transformation in order to accommodate the rise in $S_{T}$ or, equivalently, in the rate of energy release per unit volume. Such qualitative change may indicate the transition from the deflagration to a detonation. Detailed discussion of this nonsteady regime will be presented in a separate paper.

Acknowledgments We are deeply grateful to Forman Williams for numerous valuable inputs that have greatly improved this paper. We also thank Craig Wheeler, Vadim Gamezo, and James Driscoll for stimulating discussions. This work was supported in part by the Naval Research Laboratory, the Office of Naval Research, the Air Force Office of Scientific Research under the grant F1ATA09114G005, and by the National Science Foundation through TeraGrid resources provided by NCSA and TACC under the grant TG-AST080006N. Additional computing facilities were provided by the Department of Defense High Performance Computing Modernization Program. We also gratefully acknowledge the hospitality and support of the Nordic Institute for Theoretical Physics, and in particular of Axel Brandenburg, during the "Turbulent Combustion" program where parts of this work were done. 
Table 1. Nomenclature

\begin{tabular}{|c|c|}
\hline$A_{Y} \equiv A(Y)$ & surface area of the $Y$ isosurface \\
\hline$A_{L}$ & surface area of laminar flame \\
\hline$A_{T} \equiv A_{0.15}$ & surface area of turbulent flame \\
\hline$B$ & pre-exponential factor \\
\hline$C_{p}$ & specific heat at constant pressure \\
\hline$D$ & molecular diffusion coefficient (eq.7) \\
\hline$D_{0}$ & molecular diffusion constant \\
\hline$D_{T}$ & turbulent diffusion coefficient (eq. 2) \\
\hline$D a$ & Damköhler number, $\left(l / U_{l}\right) /\left(l_{F} / S_{L, 0}\right)$ \\
\hline$I$ & stretch factor (eq.1) \\
\hline$I_{Y}$ & ratio of $S_{T} / S_{L}$ to $A_{Y} / L^{2}$ (eq. 16 \\
\hline$K a$ & Karlovitz number, $\left(l_{F} / l\right)^{1 / 2}\left(U_{l} / S_{L}\right)^{3 / 2}$ \\
\hline$K a^{\text {crit }}$ & critical Karlovitz number for cusp formation (eq. 33 \\
\hline$l$ & integral scale \\
\hline$l_{c}$ & characteristic cusp length \\
\hline$l_{F}$ & full width of laminar flame, $\approx 2 \delta_{L, 0}$ \\
\hline$L$ & domain width, energy-injection scale \\
\hline$L_{G}$ & Gibson scale, $l\left(S_{L, 0} / U_{l}\right)^{3}$ \\
\hline Le & Lewis number, $\mathcal{K} / D$ \\
\hline$M$ & molecular weight \\
\hline$M a_{F}$ & Mach number in fuel, $U\left(\gamma P_{0} / \rho_{0}\right)^{-1 / 2}$ \\
\hline$M a_{P}$ & Mach number in product, $U\left(\gamma P_{0} / \rho_{P, 0}\right)^{-1 / 2}$ \\
\hline$n$ & Temperature exponent \\
\hline$P_{0}$ & initial fuel pressure \\
\hline$q$ & chemical energy release \\
\hline$Q$ & activation energy \\
\hline$S_{L}$ & actual instantaneous laminar flame speed \\
\hline$S_{L, 0}$ & initial laminar flame speed \\
\hline$S_{n}$ & local flame burning speed \\
\hline$S_{n}^{*}$ & maximum local burning speed in the cusp \\
\hline$S_{T}^{n}$ & turbulent flame speed \\
\hline$t$ & time \\
\hline$t_{i g n}$ & time of ignition \\
\hline$t_{\text {total }}$ & total simulation time from $t_{i g n}$ \\
\hline$T_{0}$ & initial fuel temperature \\
\hline$T_{P, 0}$ & initial post-flame temperature \\
\hline $\mathbf{u}=\left(u_{x}, u_{y}, u_{z}\right)$ & flow velocity \\
\hline$U$ & turbulent velocity at scale $L$ \\
\hline$U_{c}$ & speed of cusp propagation (eq. 23 \\
\hline$U_{l}$ & integral velocity \\
\hline$U_{l}^{c r i t}$ & critical value of $U_{l}$ for cusp formation (eq. 32) \\
\hline$U_{r m s}$ & turbulent r.m.s. velocity \\
\hline$U_{\delta}$ & turbulent velocity at scale $\delta_{L, 0}$ \\
\hline$V_{D}$ & diffusion velocity \\
\hline $\mathbf{x}=(x, y, z)$ & spatial coordinate in the domain \\
\hline$Y$ & fuel mass fraction \\
\hline$\dot{Y}$ & reaction rate (eq. 8 \\
\hline$z_{0, \min }$ & left $z$-boundary of the flame brush (eq. 10 \\
\hline$z_{1, \max }$ & right $z$-boundary of the flame brush (eq. 10 ) \\
\hline
\end{tabular}


Table 1 (cont'd)

\begin{tabular}{ll}
\hline$z_{T, 0}$ & initial flame position along $z$-axis \\
$\alpha$ & inclination angle between planar flame sheets \\
$\gamma$ & adiabatic index \\
$\delta_{L}$ & actual instantaneous thermal width of laminar flame \\
$\delta_{L, 0}$ & initial thermal width of laminar flame \\
$\delta_{T}$ & turbulent flame width \\
$\Delta x$ & cell size \\
$\Delta t_{c}$ & time lag of maximum cross-correlation \\
$\eta$ & Kolmogorov scale \\
$\varepsilon$ & energy-injection rate \\
$\kappa_{0}$ & thermal conduction constant \\
$\mathcal{K}$ & thermal conduction coefficient (eq.7 \\
$\rho_{0}$ & initial fuel density \\
$\rho_{P, 0}$ & initial post-flame density \\
$\Sigma_{Y} \equiv \Sigma(Y)$ & surface density of the $Y$ isosurface (eq. 14 \\
$\Sigma_{T} \equiv \Sigma_{0.15}$ & surface density of turbulent flame \\
$\tau_{e d}$ & eddy turnover time, $L / U$ \\
\hline$(\ldots)$ & time averaging \\
\hline
\end{tabular}

Table 2. Input model parameters and resulting computed laminar flame properties

\begin{tabular}{cc}
\hline & Input \\
$T_{0}$ & $293 \mathrm{~K}$ \\
$P_{0}$ & $1.01 \times 10^{6} \mathrm{erg} / \mathrm{cm}^{3}$ \\
$\rho_{0}$ & $8.73 \times 10^{-4} \mathrm{~g} / \mathrm{cm}^{3}$ \\
$\gamma$ & 1.17 \\
$M$ & $21 \mathrm{~g} / \mathrm{mol}^{3}$ \\
$B$ & $6.85 \times 10^{12} \mathrm{~cm}^{3} /(\mathrm{g} \mathrm{s})$ \\
$Q$ & $46.37 \mathrm{RT}_{0}$ \\
$q$ & $43.28 \mathrm{RT}_{0} / \mathrm{M}$ \\
$\kappa_{0}$ & $\left.2.9 \times 10^{-5} \mathrm{~g} /(\mathrm{s} \mathrm{cm} \mathrm{K})^{n}\right)$ \\
$D_{0}$ & $\left.2.9 \times 10^{-5} \mathrm{~g} /(\mathrm{s} \mathrm{cm} \mathrm{K})^{\mathrm{n}}\right)$ \\
$n$ & 0.7 \\
& Output \\
$T_{P, 0}$ & $2135 \mathrm{~K}$ \\
$\rho_{P, 0}$ & $1.2 \times 10^{-4} \mathrm{~g} / \mathrm{cm}^{3}$ \\
$\delta_{L, 0}$ & $0.032 \mathrm{~cm}$ \\
$S_{L, 0}$ & $302 \mathrm{~cm} / \mathrm{s}$ \\
\hline
\end{tabular}


Table 3. Parameters of simulations ${ }^{\mathrm{a}}$

\begin{tabular}{|c|c|c|c|}
\hline & S1 & S2 & S3 \\
\hline Mesh & $64 \times 64 \times 1024$ & $128 \times 128 \times 2048$ & $256 \times 256 \times 4096$ \\
\hline$L$ & & $0.259 \mathrm{~cm}=8 \delta_{L, 0}$ & \\
\hline$\Delta x$ & $4.05 \times 10^{-3} \mathrm{~cm}$ & $2.02 \times 10^{-3} \mathrm{~cm}$ & $1.01 \times 10^{-3} \mathrm{~cm}$ \\
\hline$\delta_{L, 0} / \Delta x$ & 8 & 16 & 32 \\
\hline$z_{T, 0}$ & & $1.95 \mathrm{~cm}=7.52 L$ & \\
\hline$\varepsilon$ & & $1.26 \times 10^{9} \mathrm{erg} /\left(\mathrm{cm}^{3} \mathrm{~s}\right)$ & \\
\hline$U_{\delta}$ & & $4.53 \times 10^{3} \mathrm{~cm} / \mathrm{s}=15 S_{L, 0}$ & \\
\hline$U$ & & $9.07 \times 10^{3} \mathrm{~cm} / \mathrm{s}=30 S_{L, 0}$ & \\
\hline$U_{r m s}$ & & $1.04 \times 10^{4} \mathrm{~cm} / \mathrm{s}=34.48 S_{L, 0}$ & \\
\hline$U_{l}$ & & $5.60 \times 10^{3} \mathrm{~cm} / \mathrm{s}=18.54 S_{L, 0}$ & \\
\hline$l$ & & $6.04 \times 10^{-2} \mathrm{~cm}=1.87 \delta_{L, 0}$ & \\
\hline$\tau_{e d}$ & & $2.86 \times 10^{-5} \mathrm{~s}$ & \\
\hline$t_{i g n}$ & $3.0 \tau_{e d}$ & $3.0 \tau_{e d}$ & $2.0 \tau_{e d}$ \\
\hline$t_{\text {total }}$ & & $16.0 \tau_{e d}$ & \\
\hline$D a$ & & 0.05 & \\
\hline$L_{G}$ & & $9.47 \times 10^{-6} \mathrm{~cm}=2.96 \times 10^{-4} \delta_{L, 0}$ & \\
\hline$M a_{F}$ & & 0.25 & \\
\hline$M a_{P}$ & & 0.09 & \\
\hline
\end{tabular}

a Parameters common to all simulations are shown only once in S2 column.

Table 4. Time-averaged properties of the turbulent flame brush ${ }^{\mathrm{a}}$

\begin{tabular}{ccccccccccc}
\hline \hline & $\overline{\delta_{T} / \delta_{L}}$ & $O\left(\overline{\delta_{T} / \delta_{L}}\right)$ & $\overline{S_{T} / S_{L}}$ & $O\left(\overline{S_{T} / S_{L}}\right)$ & $\bar{A}_{0.15} / L^{2}$ & $O\left(\bar{A}_{0.15} / L^{2}\right)$ & $\bar{\Sigma}_{0.15}, \mathrm{~mm}^{-1}$ & $O\left(\bar{\Sigma}_{0.15}\right)$ & $\bar{I}_{0.15}$ & $O\left(\bar{I}_{0.15}\right)$ \\
\hline S1 & 20.03 & & 5.19 & & 3.91 & & 0.74 & & 1.31 & \\
S2 & 17.39 & 2.21 & 3.91 & 2.19 & 3.23 & 2.84 & 0.67 & 4.07 & 1.20 & 1.50 \\
S3 & 16.66 & & 3.55 & & 3.12 & & 0.67 & & 1.14 & \\
\hline
\end{tabular}

\footnotetext{
${ }^{\text {a }}$ Time-averaging for all variables is performed over the time interval $\left[2 \tau_{e d}-16 \tau_{e d}\right]$.
} 


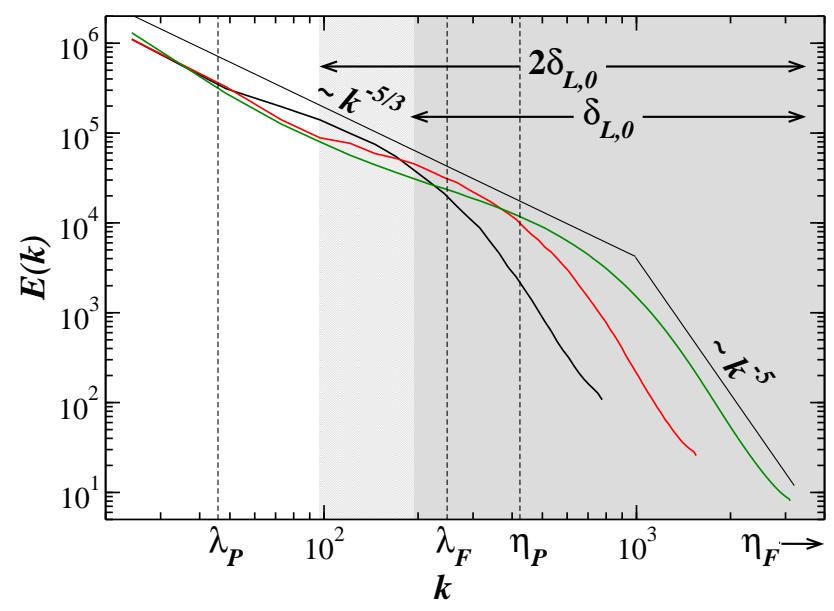

Figure 1 Instantaneous kinetic energy spectral density for simulations S1 (black), S2 (red), and S3 (green) at a time immediately prior to ignition. Shaded regions illustrate scales associated with thermal width $\delta_{L, 0}$ and full width $2 \delta_{L, 0}$ of the laminar flame. Vertical dashed lines show wavenumbers corresponding to the Taylor microscales in the product, $\lambda_{P}$, and fuel, $\lambda_{F}$, as well as the Kolmogorov scale in the product, $\eta_{P}$, based on the value of the viscosity coefficient corresponding to the $S c=P r=1$ condition. The wavenumber corresponding to the Kolmogorov scale in the fuel, $\eta_{F}=1.18 \times 10^{-3} \mathrm{~cm}$, is located outside the range of the graph. (Reproduced from [11].)

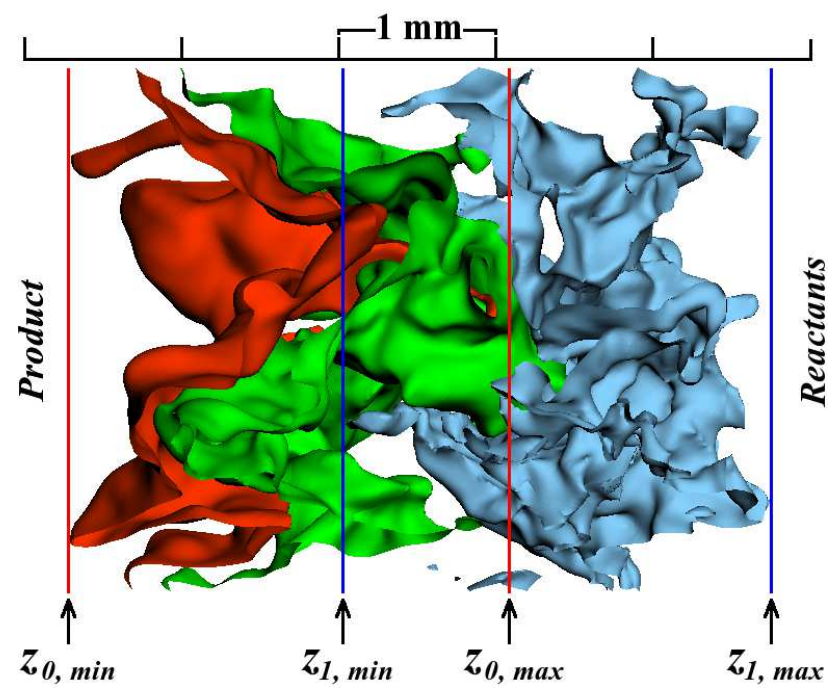

Figure 2 Isosurfaces of $Y$ in simulation S2 at $t=13 \tau_{e d}$ (cf. Fig.3, middle row, left panel in [11]). Isosurface values are 0.05 (red), 0.6 (green), 0.95 (blue). Red and green isosurfaces bound the reaction zone of the flame. Green and blue isosurfaces bound the preheat zone. The $z_{0, \min }$ and $z_{1, \text { max }}$ mark the flame-brush bounds. The $z_{0, \max }$ and $z_{1, \min }$ indicate, respectively, the maximum extents of product and fuel penetration into the flame brush (see Appendix A for further discussion of $z_{0, \max }$ and $z_{1, \min }$ ). (Reproduced from [11].) 

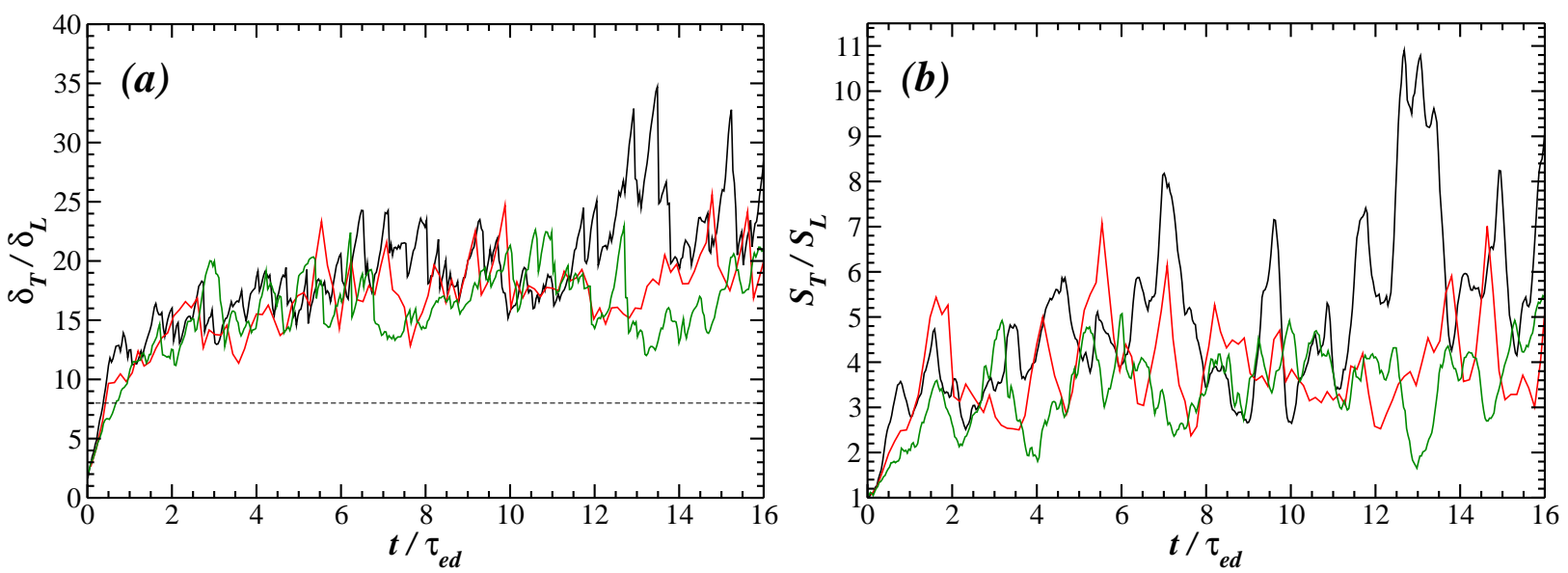

Figure 3 (a) Evolution of the normalized turbulent flame width $\delta_{T} / \delta_{L}$. Note that domain width $L=8 \delta_{L, 0} \approx 8 \delta_{L}$ indicated with the horizontal dashed line. (b) Evolution of the normalized turbulent flame speed $S_{T} / S_{L}$. In both panels: black lines correspond to simulation S1, red to S2, and green to S3. (cf. Fig. 4 in [11].) 

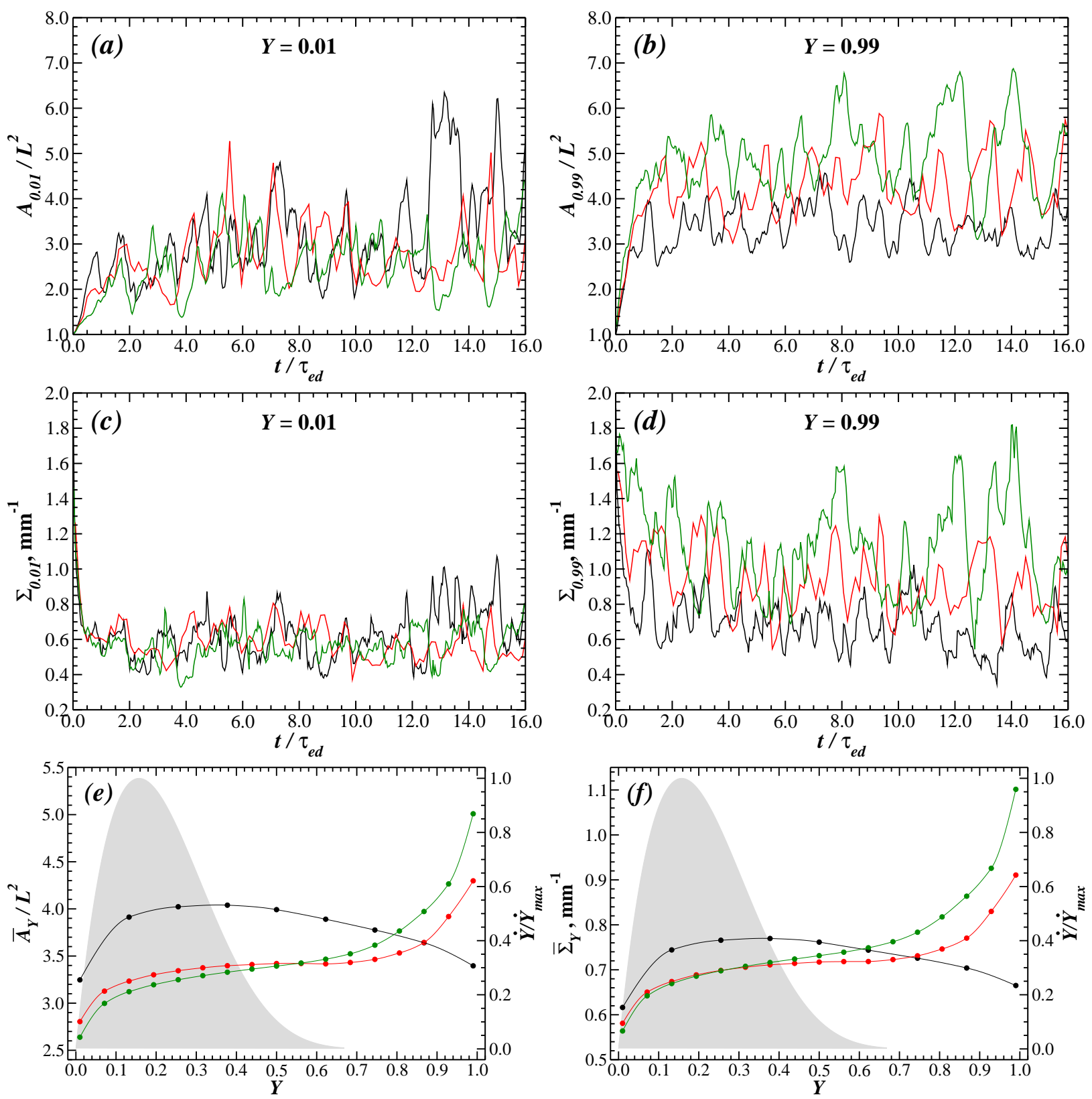

Figure 4 Evolution of the normalized flame surface area (a) $A_{0.01} / L^{2}$ and (b) $A_{0.99} / L^{2}$. Evolution of the flame surface density (c) $\Sigma_{0.01}$ and (d) $\Sigma_{0.99}$. (e) Time-averaged normalized isosurface area $\bar{A}_{Y} / L^{2}$. (f) Time-averaged isosurface density $\bar{\Sigma}_{Y}$. In panels (e) and (f) time averaging is performed over the time interval $\left[2 \tau_{e d}-16 \tau_{e d}\right]$, circles represent calculated values and solid lines are the Akima spline fits. Shaded gray region shows the distribution of the reaction rate, $\dot{Y}$, in the exact laminar flame solution normalized by its peak value $\dot{Y}_{\max }=9.5 \times 10^{4} \mathrm{~s}^{-1}$. In all panels: black corresponds to simulation $\mathrm{S} 1$, red to $\mathrm{S} 2$, and green to $\mathrm{S} 3$. 

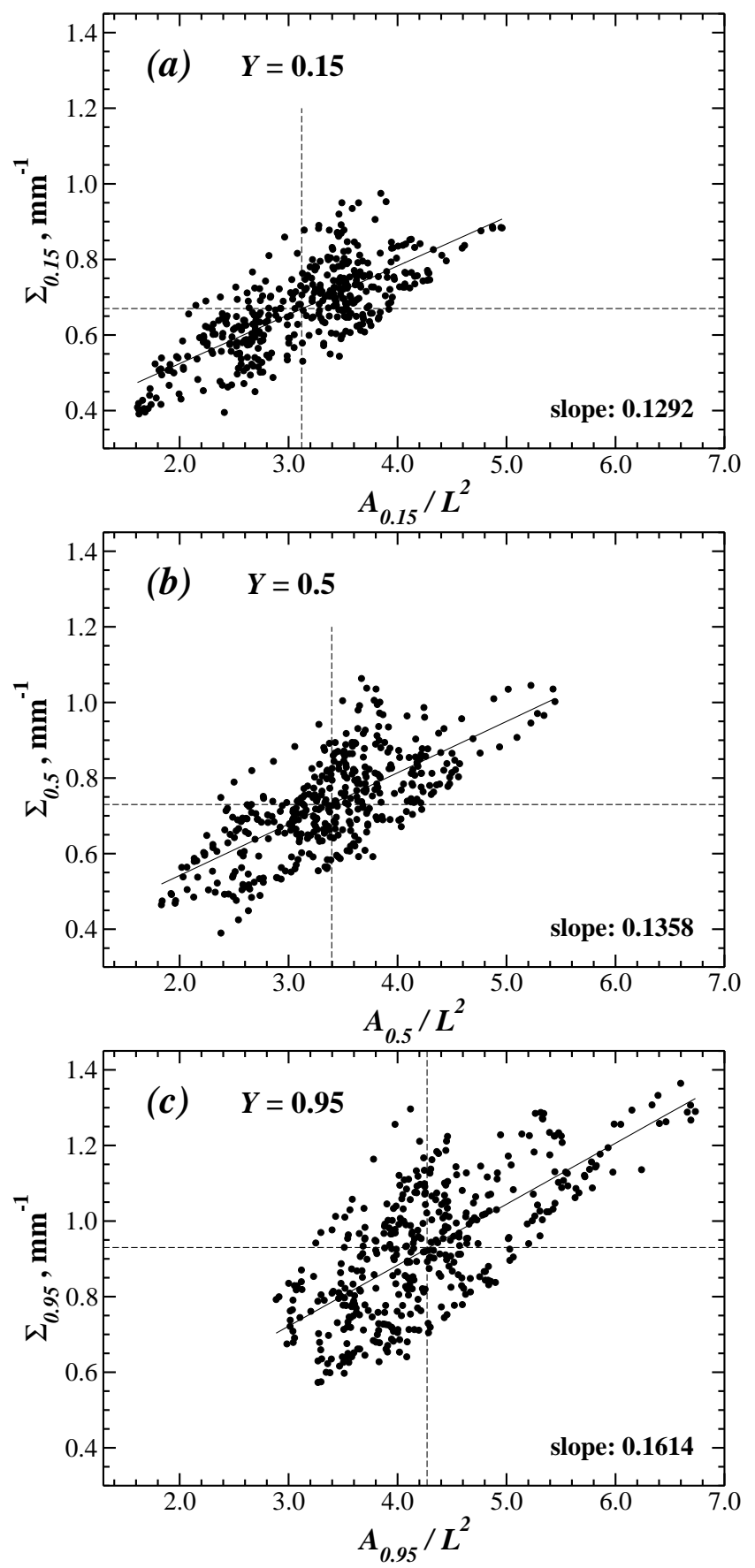

Figure 5 Correlation between $\Sigma_{Y}$ and $A_{Y} / L^{2}$ in simulation S3 for three values of $Y$. Graphs exclude data from the time interval $\left[0-2 \tau_{e d}\right]$ during which the turbulent flame develops its equilibrium state. Dashed lines show time-averaged values of $\Sigma_{Y}$ and $A_{Y} / L^{2}$. Solid lines show the least squares fit with its slope given in each panel. 

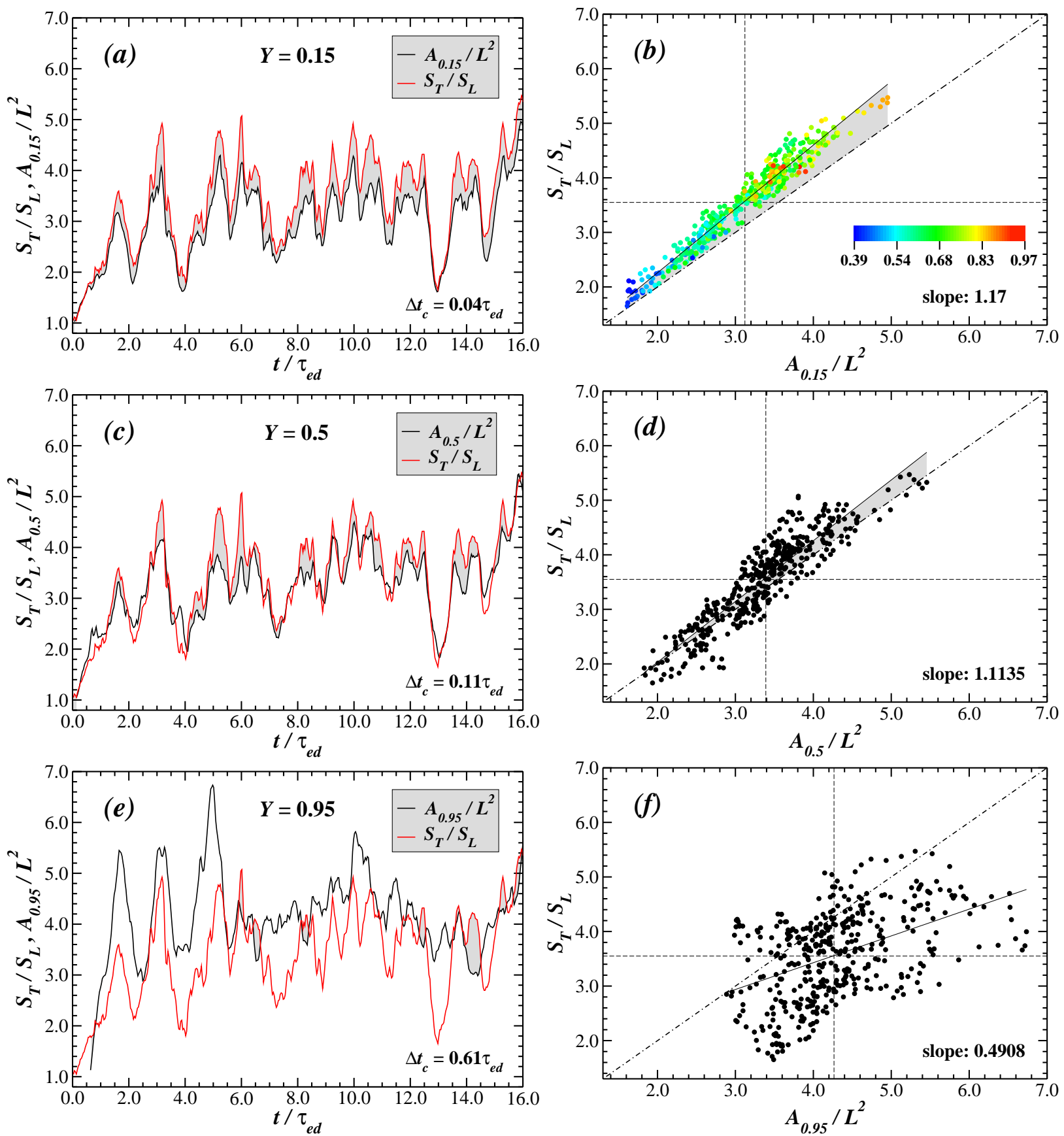

Figure 6 Correlation between $A_{Y} / L^{2}$ and $S_{T} / S_{L}$ in simulation S3. $A_{Y}$ is considered for three values of $Y$ : $(\mathrm{a}, \mathrm{b})$ $Y=0.15$, (c, d) $Y=0.5$, and (e, f) $Y=0.95$. In panels (a), (c), and (e) $A_{Y} / L^{2}$ is shifted in time to the right by the time lag $\Delta t_{c}$ shown in each panel. Graphs in panels (b), (d), and (f) are constructed based on the data shown, respectively, in panels (a), (c), and (e) excluding the time $\left[0-2 \tau_{e d}\right]$ during which the turbulent flame develops its equilibrium state. In panel (b) each data point is colored according to the corresponding value of $\Sigma_{0.15}$ with the color scale given in units $\mathrm{mm}^{-1}$ (cf. Fig. 5a). In panels (b), (d), and (f) dashed lines show time-averaged values of $A_{Y} / L^{2}$ and $S_{T} / S_{L}$, solid lines show the least squares fit with its slope given in each panel, and dash-dot lines correspond to $S_{T} / S_{L}=A_{Y} / L^{2}$. Shaded gray regions in panels (a)-(e) illustrate the exaggerated response of $S_{T}$ to the increase of $A_{Y}$. 

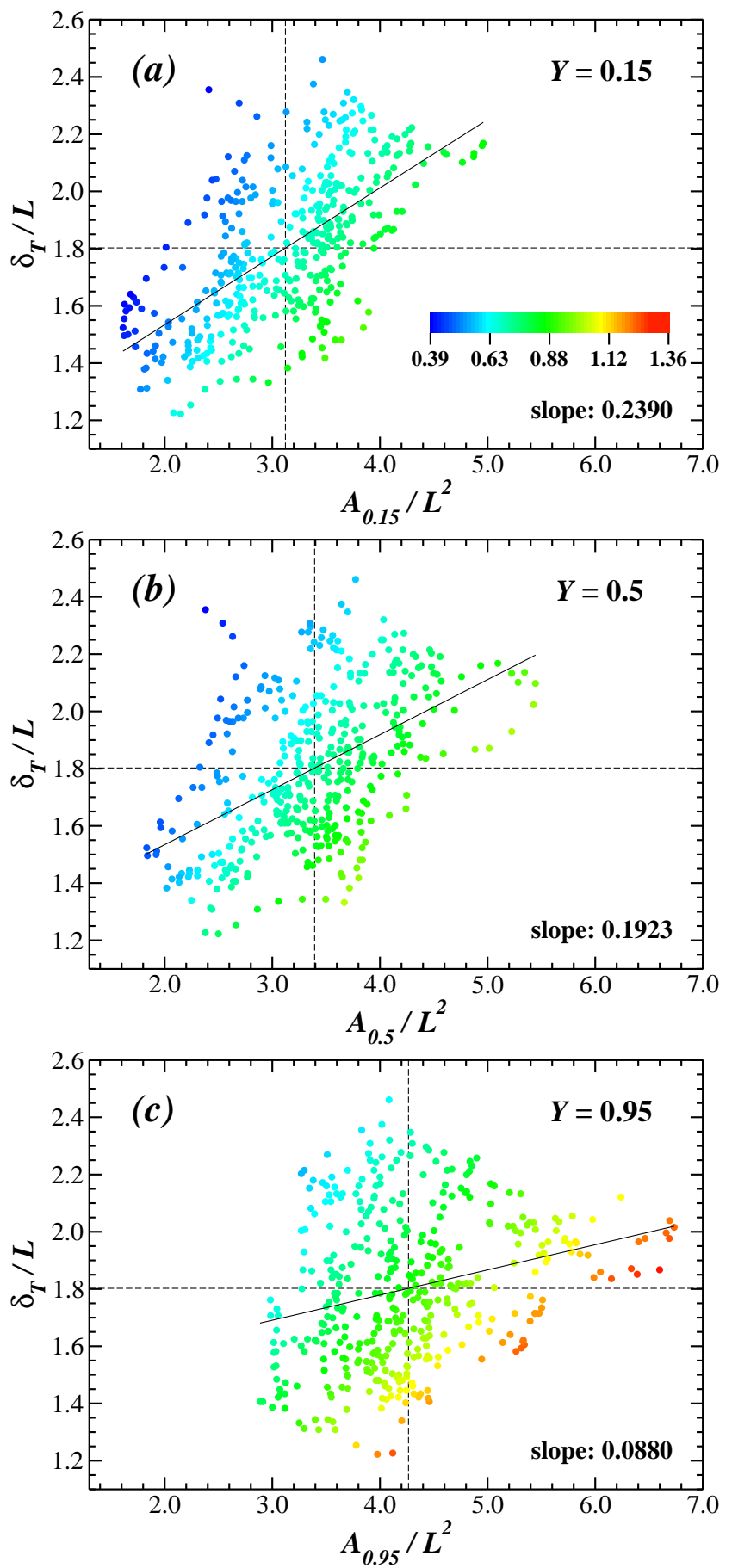

Figure 7 Correlation between $A_{Y} / L^{2}$ and $\delta_{T} / L$ in simulation S3 for three values of $Y$. Graphs exclude data from the time interval $\left[0-2 \tau_{e d}\right]$ during which the turbulent flame develops its equilibrium state. Dashed lines show timeaveraged values of $A_{Y} / L^{2}$ and $\delta_{T} / L$. Solid lines show the least squares fit with its slope given in each panel. Each data point is colored according to the corresponding value of $\Sigma_{Y}$ with the common color scale in units $\mathrm{mm}^{-1}$ shown in panel (a) (cf. Fig. 5). 


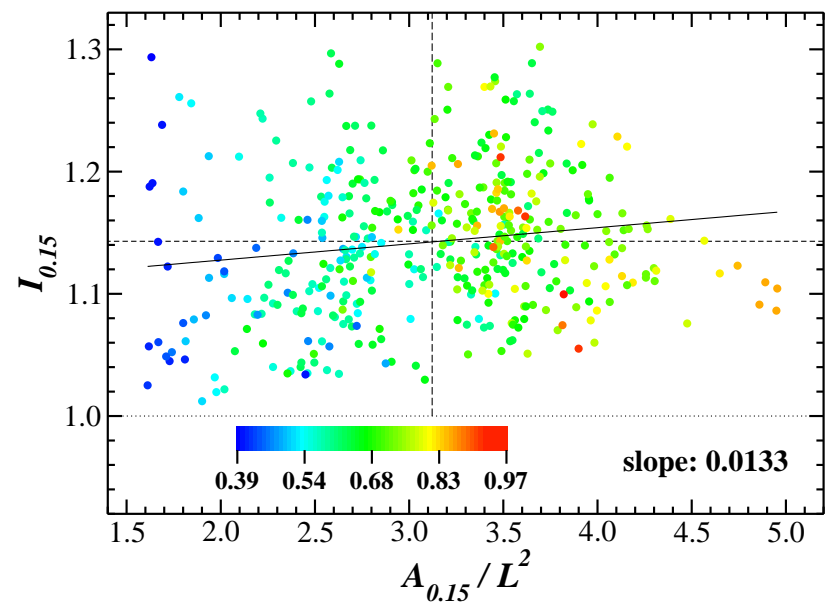

Figure 8 Instantaneous values of $I_{0.15}$ as a function of $A_{0.15} / L^{2}$ in simulation $S 3$. Graph is constructed from data shown in Fig. 6 b. Each data point is colored according to the corresponding value of $\Sigma_{0.15}$ with the color scale given in units $\mathrm{mm}^{-1}$. Dashed lines show time-averaged values. The solid line gives the least squares fit with its slope given in the panel.

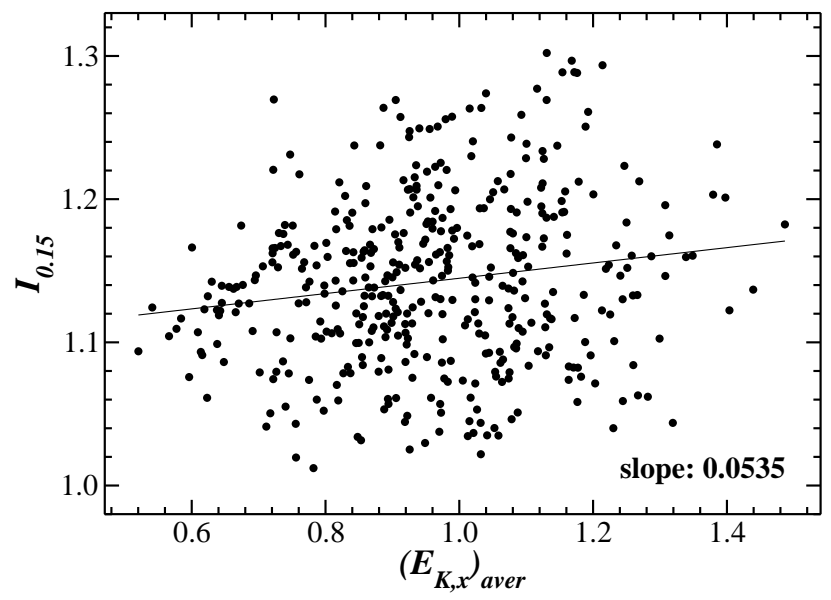

Figure 9 Instantaneous values of $I_{0.15}$ in simulation S3 as a function of the volume-averaged normalized $x$-component of the kinetic energy inside the flame brush and in the fuel immediately ahead of it. The solid line shows the least squares fit with its slope given in the panel. See text for further details. 


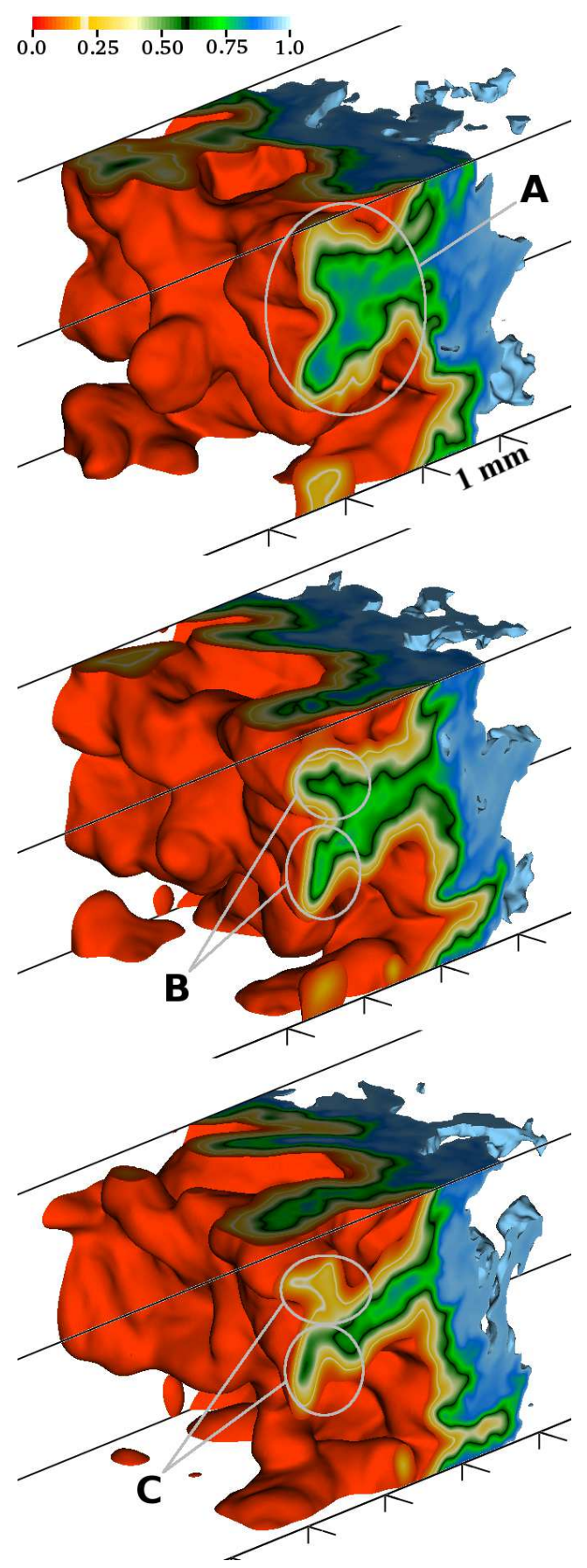

Figure 10 Flame collision and the formation of cusps in the turbulent flame. Shown is the flame-brush structure based on the isovolume of $Y$ in simulation S3. Bounding isosurfaces represent $Y=0.05$ and $Y=0.95$ and the flame brush is shown from the product side. Upper panel corresponds to the time $t=11.86 \tau_{e d}$, while the middle and lower panels show the flame structure, respectively, $0.1 \tau_{e d}=3 \mu \mathrm{s}$ and $0.2 \tau_{e d}=6 \mu \mathrm{s}$ later. The thin black line, corresponding to $Y=0.6$, marks the boundary between the preheat and reaction zones. The thin white line, corresponding to $Y=0.2$, shows the location of the peak reaction rate. Regions A, B, and C show the three main stages of the flame collision and the formation of a cusp discussed in $\S 6.1$. Note also two elongated regions of flame collision forming near the upper face of the domain. 

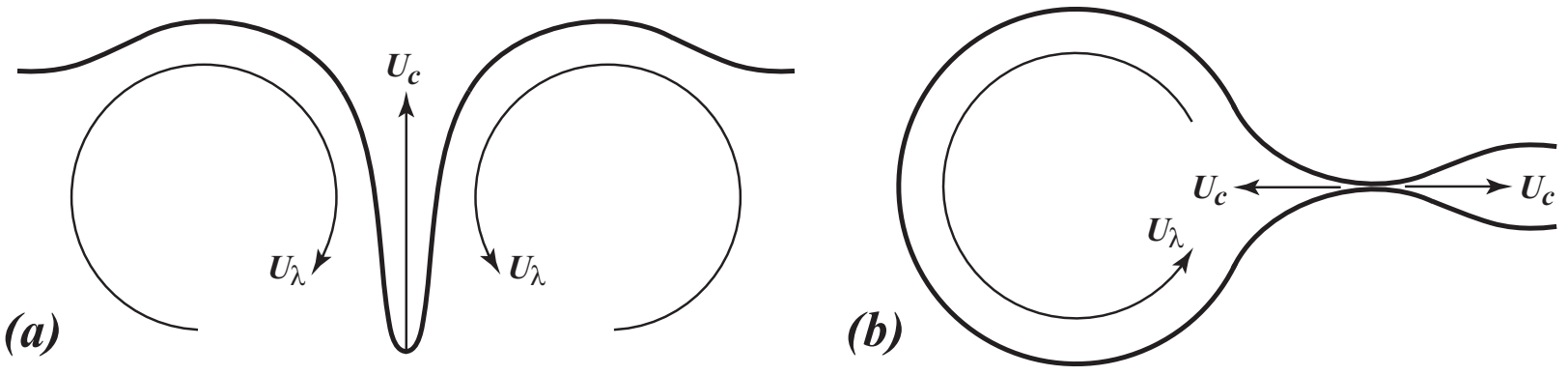

Figure 11 Schematic of the two main channels of cusp formation. (a) Local flame collisions. Turbulent motions with velocity $U_{\lambda}$ on the scale $\lambda$ fold the flame, gradually increasing its surface curvature at a specific point until it becomes $\sim 1 / \delta_{L}$. (b) Nonlocal flame collisions. The flame is folded by turbulent motions on a larger scale. The curvature of the flame surface remains small at all points until the collision of the two flame sheets causes an abrupt formation of two cusps. Resulting cusps propagate in both cases with the phase velocity $U_{c}$, which depends on the local flame curvature. See text for further details.

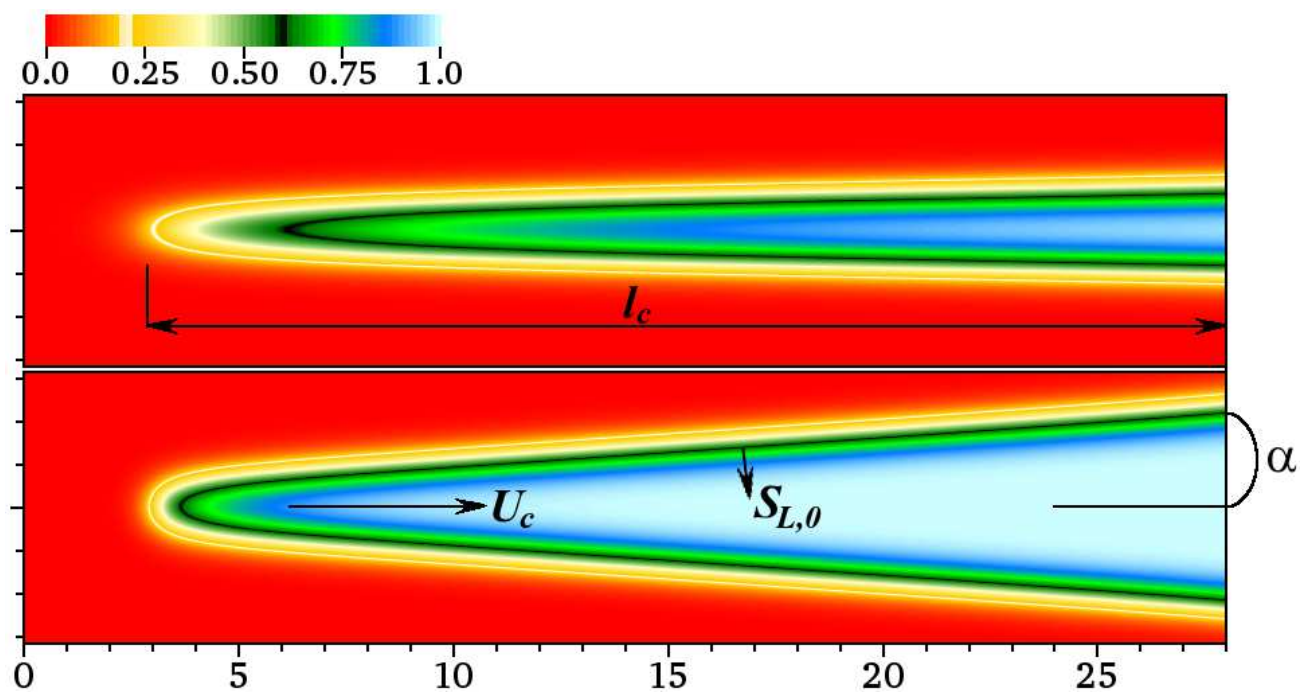

Figure 12 Structure of a cusp formed by the collision of two planar flame sheets. Shown is the distribution of $Y$ for the flame inclination angle $\alpha=1^{\circ}$ (upper panel) and $\alpha=4^{\circ}$ (lower panel). Scale of the panel axes is given in units of $\delta_{L, 0}$. Away from the cusp tip, the flame propagates in the direction normal to its surface with the laminar flame speed, $S_{L, 0}$, causing the tip to move to the right with the speed $U_{c}$. Also shown is the variable length, $l_{c}$, of the collision region (see text). Thin black line marks the boundary between the reaction and preheat zones, while the thin white line indicates the region of peak reaction rate. Note the substantial broadening of the flame, and thus the reaction zone, near the tip of the cusp at $\alpha=1^{\circ}$ compared to $\alpha=4^{\circ}$ (cf. Fig. 10). 

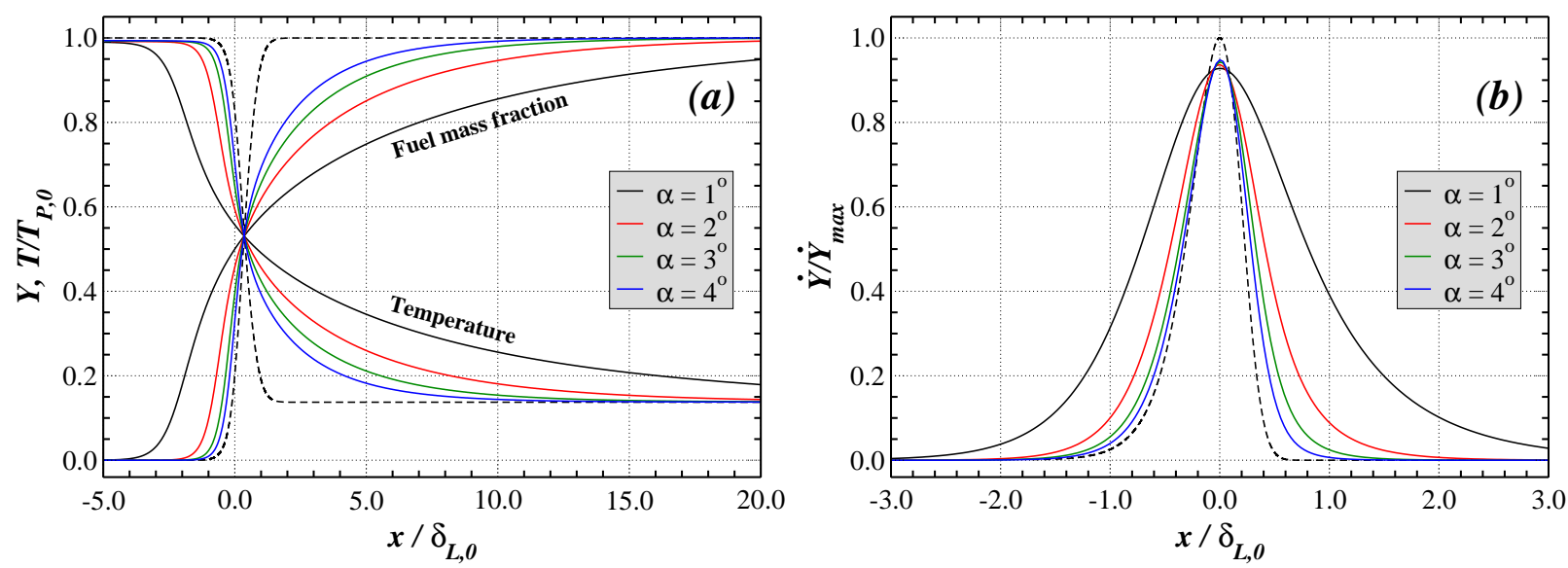

Figure 13 Structure of a cusp formed by the collision of two planar flame sheets for four flame inclination angles, $\alpha$. Shown are distributions of (a) $Y$ and $T$, as well as (b) the reaction rate, $\dot{Y}$, along the symmetry line of the cusp (cf. Fig. 12). Dashed lines in both panels indicate the exact planar laminar flame solution. Profiles of $T$ and $\dot{Y}$ are normalized by their respective maximum values in the exact laminar solution, i.e., $T_{P, 0}$ (see Table 2 ) and $\dot{Y}_{\max }=$ $9.5 \times 10^{4} \mathrm{~s}^{-1}$
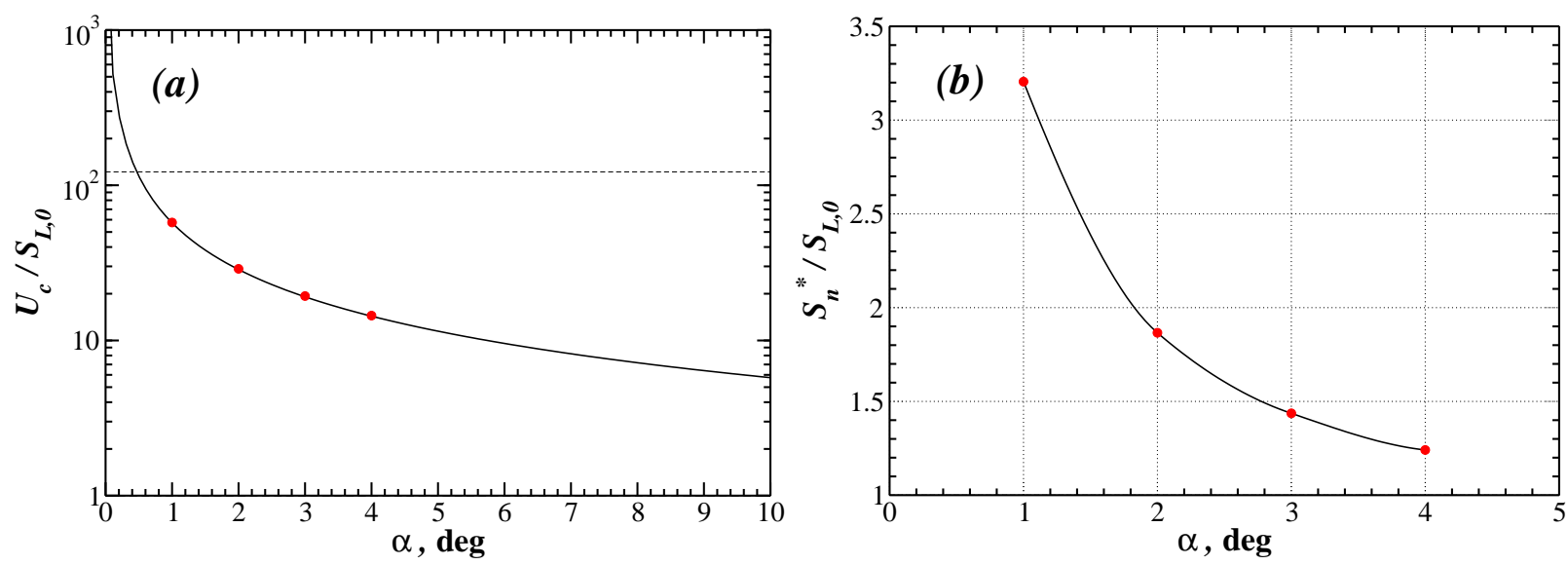

Figure 14 (a) Dependence of the normalized speed of cusp propagation, $U_{c} / S_{L, 0}$, on the flame inclination angle, $\alpha$. Solid line corresponds to the analytic expression given in eq. (23), red circles show the computed values. Horizontal dashed line indicates sound speed in cold fuel. (b) Dependence of the maximum normalized local flame speed in the cusp, $S_{n}^{*} / S_{L, 0}$, given by eq. (24), on $\alpha$. Red circles show the computed values, solid line is the Akima spline fit. 


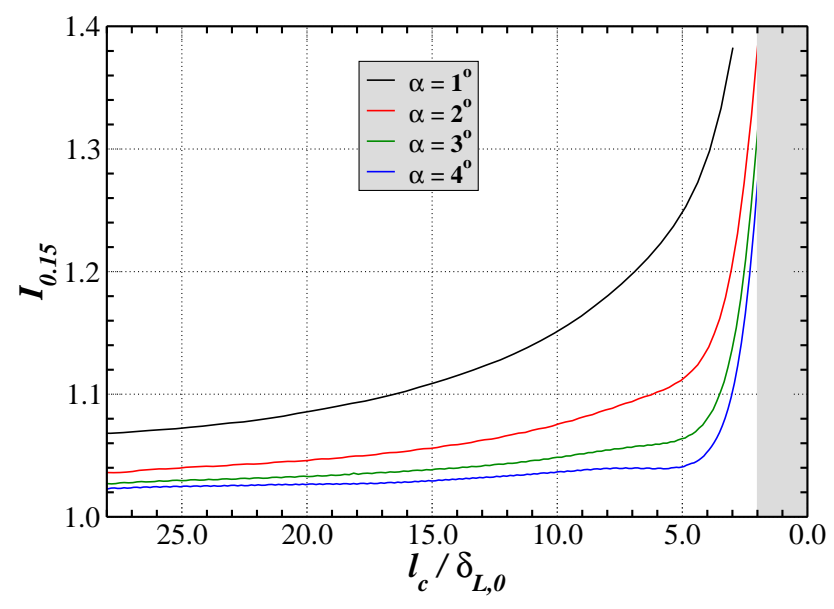

Figure 15 Dependence of $I_{0.15}$, given by eq. (26), on the normalized length of the flame-collision region, $l_{c} / \delta_{L, 0}$, for four values of $\alpha$. The shaded gray area indicates the full laminar flame width $l_{F} \approx 2 \delta_{L, 0}$. See text for further details.

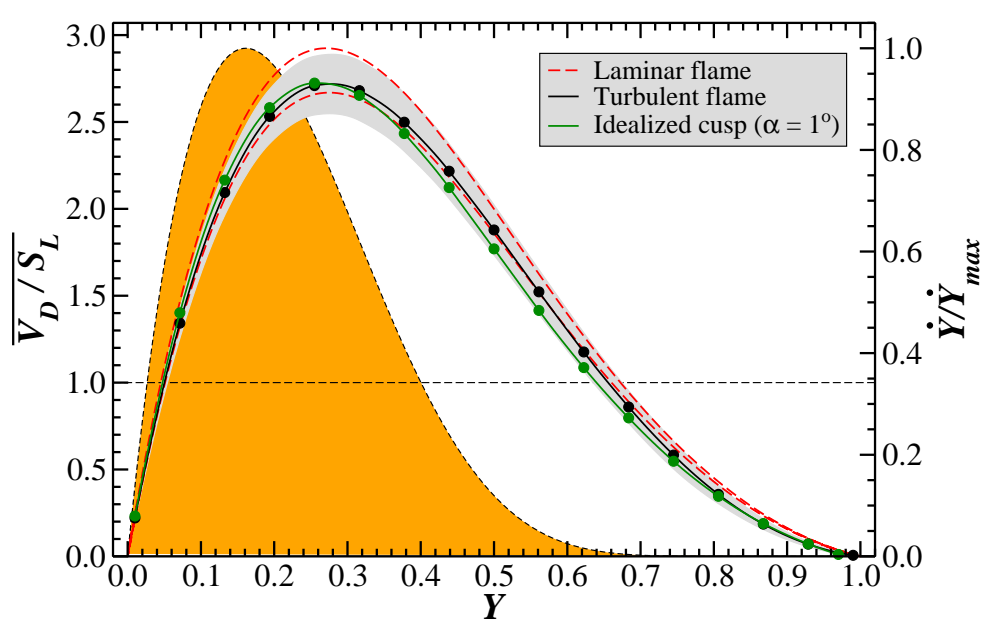

Figure 16 Time-averaged distributions of the normalized diffusion velocity in simulation S3 (black line) and in the idealized 2D cusp at $\alpha=1^{\circ}$ shown in the upper panel of Fig. 12 (green line). Circles are calculated values, solid lines are the Akima spline fits. The shaded gray region is the $1 \sigma$ standard deviation of the instantaneous values of $V_{D} / S_{L}$ in S3. Dashed red lines are the exact laminar flame solutions corresponding to fuel temperatures $T_{0}=293 \mathrm{~K}$ (upper line) and $320 \mathrm{~K}$ (lower line). The shaded orange region shows the distribution of the reaction rate, $\dot{Y}$, in the exact laminar flame solution (fuel temperature $T_{0}$ ) normalized by its peak value $\dot{Y}_{\max }=9.5 \times 10^{4} \mathrm{~s}^{-1}$. See text for further details. 


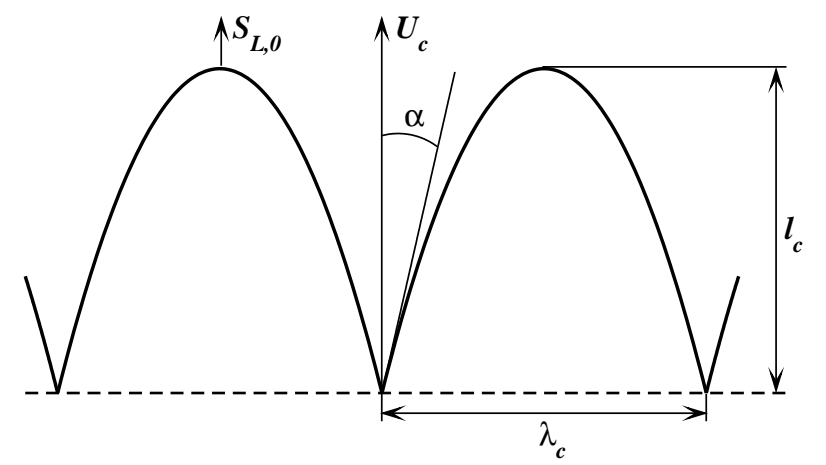

Figure 17 Illustration of the idealized perturbed flame stabilized by the propagation of a cusp with the speed $U_{c}$ (cf. Figs. 117 and 12.

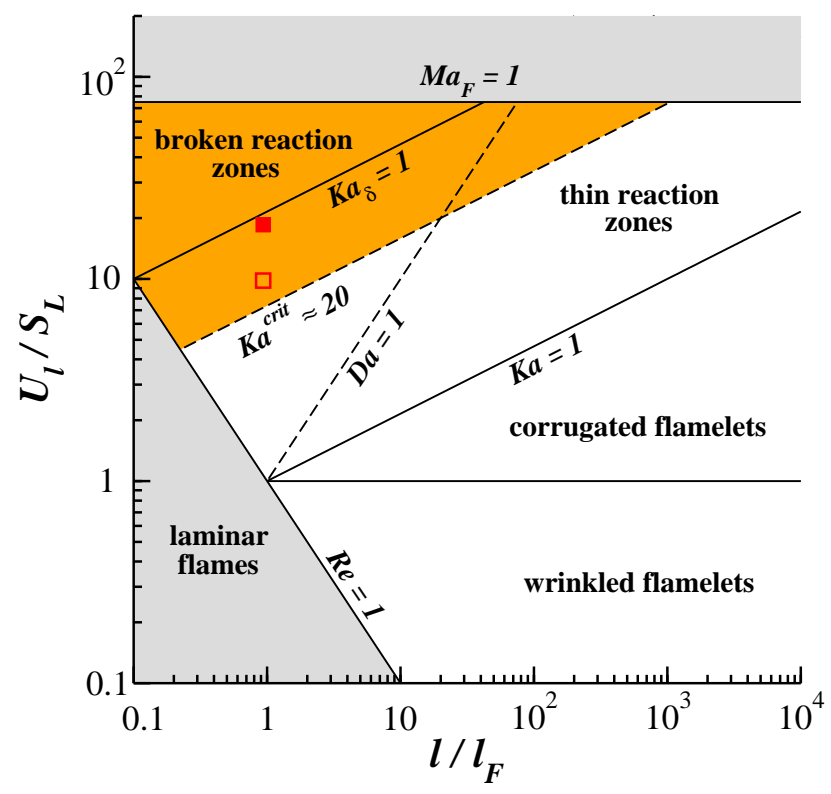

Figure 18 Combustion regime diagram according to [5]. The orange region above the $K a^{c r i t} \approx 20$ line shows, according to the criterion discussed in $\S 6.5$, the range of the regimes in which the formation of cusps is expected to substantially affect the turbulent flame speed. The solid red square corresponds to the simulation S3 presented in this work that gives $\bar{I}_{0.15} \approx 1.14$. The open red square shows the regime with one half the turbulent intensity in which the value of $\bar{I}_{0.15} \approx 1.1$ was determined. Flamelets are typically believed to exist in the regimes below the $K a_{\delta}=K a / 100=1$ line [5]. The traditional form of the diagram was also modified by adding the line $M a_{F}=1$ indicating the region of supersonic turbulence in the cold $\mathrm{H}_{2}$-air fuel under the atmospheric conditions. See text for further details. 


\section{Appendix A. Definition of the flame surface density}

In the definition of $\Sigma_{Y}$, given in eq. (14), the isosurface area, $A_{Y}$, was normalized by the total volume of the flame brush, $\delta_{T} L^{2}$. Individual isosurfaces, however, do not occupy all of this volume. For instance, this is the case for isosurfaces of $Y=0.05$ and $Y=0.95$, which are used to specify $\delta_{T}$ in eqs. (9)-(10), simply because, by definition, they are separated at least by the full flame width, $l_{F}$, or a larger distance if the flame becomes broadened. In our case, $l_{F} \approx 2 \delta_{L}$ (see Fig. 7 in [11]) is a substantial fraction of $\delta_{T}$. Consider Fig. 2. It can be seen that neither the $Y=0.05$, nor the $Y=0.95$ isosurface extend over the full width of the flame brush, and they indeed occupy a smaller volume.

In order to consider this more quantitatively, we define $z_{0, \max }$ and $z_{1, \min }$ by analogy with $z_{0, \min }$ and $z_{1, \max }$ (eq.10),

$$
\begin{aligned}
& z_{0, \text { max }}=\min (z): Y(x, y, z)>0.05 \forall\left(x, y, z>z_{0, \text { max }}\right), \\
& z_{1, \text { min }}=\max (z): Y(x, y, z)<0.95 \forall\left(x, y, z<z_{1, \text { min }}\right) .
\end{aligned}
$$

In other words, $z_{0, \max }$ is the $z$-coordinate of the rightmost cell with pure product, while $z_{1, \min }$, respectively, is the $z$ coordinate of the leftmost cell with pure fuel. Thereby, they effectively measure the furthest extent of product and fuel penetration into the flame brush. Given the definitions of $z_{0, \min }$ and $z_{1, \max }$ (eq. 10), $z_{0, \min }$ and $z_{0, \max }$ bound the volume confining the $Y=0.05$ isosurface, while $z_{1, \min }$ and $z_{1, \max }$ bound the $Y=0.95$ isosurface. If $z_{1, \min }<z_{0, \max }$, these two variables bound the region of macroscopic mixing of product and fuel in the flame brush, i.e., the region in which both pure fuel and pure product can be found. On the other hand, values $z_{0, \max } \leq z_{1, \min }$ exist only in a weakly wrinkled (nearly planar) flame. Fig. 2 provides the illustration of all four of these quantities.

Next, we define the position of the turbulent flame brush as

$$
z_{T}=\frac{z_{0, \min }+z_{1, \max }}{2} .
$$

Modified $\tilde{z}_{0, \max }$ and $\tilde{z}_{1, \min }$ are then defined as offsets of $z_{0, \max }$ and $z_{1, \min }$ with respect to $z_{T}$ normalized by $\delta_{T}$, namely

$$
\tilde{z}_{0, \text { max }}=\frac{z_{0, \text { max }}-z_{T}}{\delta_{T}}, \quad \tilde{z}_{1, \text { min }}=\frac{z_{1, \min }-z_{T}}{\delta_{T}} .
$$

Thus, $\tilde{z}_{0, \max }$ and $\tilde{z}_{1, \min }$ are the relative measure of the extent to which pure fuel and pure product penetrate into the flame brush. It follows from the definition $[\mathrm{A.3}$, that both quantities take on values in the interval $[-0.5,0.5]$. For example, in a planar laminar flame, they are constant with $\tilde{z}_{0, \max }=-0.5, \tilde{z}_{1, \min }=0.5$. In the turbulent flame brush, $\tilde{z}_{0, \max }=\tilde{z}_{1, \min }=0$ would indicate that both fuel and product reach the midpoint of the flame brush, they are confined to the left and right halves of the brush, and there is no macroscopic mixing of fuel and product. This would correspond to a weakly wrinkled flame. If $\tilde{z}_{0, \max } \approx 0.5$ and $\tilde{z}_{1, \min } \approx-0.5$, then both pure product and pure fuel can be found throughout the entire volume of the flame brush.

Fig. B.19 shows the evolution of $\tilde{z}_{0, \max }$ and $\tilde{z}_{1, \min }$ for simulations S1-S3. In all cases, both parameters exhibit fairly similar behavior oscillating around zero. As the system evolves, it undergoes recurring transitions between periods of enhanced fuel-product mixing and episodes of their near complete separation when the turbulent flame becomes more planar. The correlation between these quantities and $S_{T}$ is less prominent compared, for instance, with that between $A_{Y}$ and $S_{T}$, although it is possible to associate some peaks and troughs with the corresponding changes in $S_{T}$.

It follows from Fig. B.19that throughout the course of the simulation, both the $Y=0.05$ and $Y=0.95$ isosurfaces indeed occupy regions smaller than the full flame-brush volume. This is also the case for other isosurfaces (cf. Fig. 2). Consequently, an argument can be made that when calculating $\Sigma_{Y}$, normalization should be performed not over the full volume of the flame brush, but rather over the respective volume bounding a given isosurface. For instance, for $Y=0.05$, this volume is $\left(z_{0, \text { max }}-z_{0, \min }\right) L^{2}=\left(\tilde{z}_{0, \max }+0.5\right) \delta_{T} L^{2}$, which would introduce an additional factor $1 /\left(\tilde{z}_{0, \max }+0.5\right)$ in eq. (14). Such definition of $\Sigma_{Y}$ would be a more accurate measure of how tightly a given isosurface is folded. Fig. B.19] shows that, on average, $\tilde{z}_{0, \max } \approx 0$ and, thus, the $Y=0.05$ isosurface is confined to about half of the total volume of the flame brush. Therefore, such modified definition would result in approximately twice higher values of $\Sigma_{Y}$. Ultimately, however, we are interested in determining how the overall flame, rather than individual isosurfaces, is folded inside the flame brush since only the flame as a whole has actual physical significance. Consequently, the uniform normalization over the total volume occupied by the flame appears to be a more physically grounded choice.

Further motivation for our choice of normalization in the definition (14) of $\Sigma_{Y}$ comes when we consider how $\tilde{z}_{0, \max }$ 
and $\tilde{z}_{1, \min }$ would change for larger system sizes. Since the $Y=0.05$ and $Y=0.95$ isosurfaces are always separated at least by the full width of the flame, $l_{F}$, then

$$
z_{1, \text { max }}-z_{0, \text { max }} \gtrsim l_{F}, \quad z_{1, \text { min }}-z_{0, \text { min }} \gtrsim l_{F} .
$$

Using eqs. (9), A.2), and (A.3), and the fact that in our system $l_{F} \approx 2 \delta_{L}$, inequalities (A.4) can be transformed into the following conditions which must be satisfied at each moment in time,

$$
\tilde{z}_{0, \max } \lesssim 0.5-\frac{2 \delta_{L}}{\delta_{T}}, \quad \tilde{z}_{1, \min } \gtrsim-0.5+\frac{2 \delta_{L}}{\delta_{T}}
$$

Horizontal dashed lines in Fig. B.19 show the average limiting $\tilde{z}_{0, \max }$ and $\tilde{z}_{1, \min }$ based on the values of $\overline{\delta_{T} / \delta_{L}}$ listed in Table 4 for each simulation. In particular, $\tilde{z}_{0, \max }$ must be $\lesssim 0.4$ while $\tilde{z}_{1, \min }$ must be $\gtrsim-0.4$, which closely agrees with the data shown in Fig. B.19.

It was discussed in $\S 6.6$ that $\delta_{T}$ increases with the turbulent integral scale, or, equivalently, with the system size and energy injection scale [11, 5]. It then follows from eq. (A.5) that as $\delta_{T} / \delta_{L} \rightarrow \infty$, then limiting values of $\tilde{z}_{0, \max } \rightarrow 0.5$ and $\tilde{z}_{1, \min } \rightarrow-0.5$. Therefore, in larger systems, as $\delta_{T}$ becomes large in comparison with $\delta_{L}$, the volume bounding each isosurface becomes well approximated by the total volume of the flame brush. Consequently, in the limit of large values of $\delta_{T}$, the uniform normalization for all values of $Y$ in eq. (14) becomes equivalent to the normalization by the actual volume bounding a given isosurface.

\section{Appendix B. Induction time for the planar laminar flame}

Fig. B.20 shows the distribution of induction times $\tau_{\text {ind }}$, calculated using the procedure described in $\$ 4.1$ throughout the planar laminar flame. The solid red line corresponds to the fuel temperature $T_{0}=293 \mathrm{~K}$ and density $\rho_{0}$ (see Table 2), while the dashed red line was obtained for the higher fuel temperature $320 \mathrm{~K}$ and the same density. The second temperature reflects the effect of fuel heating by turbulence in the course of the simulation $(\$ 2.2)$.

For comparison, we also show the adiabatic induction time, $\tau_{\text {ind }}^{a}$, calculated using eq. (20) based on the exact planar laminar flame solutions. Solid and dashed line correspond, respectively, to the same fuel temperatures as for $\tau_{\text {ind }}$. Overall, throughout the reaction zone, i.e., for $Y=0.2-0.6, \tau_{\text {ind }}$ and $\tau_{\text {ind }}^{a}$ differ by less than a factor of $\approx 2$. Moreover, near the peak reaction rate, i.e., at $Y \approx 0.2$, and at the boundary between the reaction and preheat zones, i.e., at $Y \approx 0.55, \tau_{\text {ind }}$ and $\tau_{\text {ind }}^{a}$ become virtually equal. In the preheat zone, however, they begin to diverge substantially. This is to be expected, since $\tau_{\text {ind }}^{a}$ considers only self-heating of a fluid element by chemical reactions and neglects the diffusive transport of heat, which is dominant in the preheat zone.

Despite the reasonable agreement in the reaction zone between $\tau_{\text {ind }}$ and $\tau_{\text {ind }}^{a}, \tau_{\text {ind }}^{a}$ should not be viewed as a model of $\tau_{\text {ind }}$, but rather as a useful approximation. In particular, Fig. B.20 shows that $\tau_{\text {ind }}^{a}$ does not capture the correct shape of the distribution of $\tau_{\text {ind }}$. The $\tau_{\text {ind }}$ increases more slowly at larger $Y$ than $\tau_{\text {ind }}^{a}$ since slower heating of a fluid element by chemical reactions is partially compensated by the larger influx of heat due to the thermal conduction. Since $\tau_{\text {ind }}^{a}$ does not account for this, its rate of growth with $Y$ is larger in the outer regions of the reaction zone. At the same time, since energy balance of a fluid element in the reaction zone is still dominated by self-heating rather than by thermal transport, $\tau_{\text {ind }}^{a}$ remains close to the actual value of the induction time given by $\tau_{\text {ind }}$.

We also show in Fig. B.20 the characteristic reaction time, $\tau_{\text {reac }}$, defined based on the reaction rate (eq. 8) and neglecting the change in the fuel concentration,

$$
\tau_{\text {reac }}\left(Y^{\prime}\right)=\frac{1}{\rho^{\prime} B} \exp \left(\frac{Q}{R T^{\prime}}\right)
$$

Similarly to $\tau_{\text {ind }}^{a}, T^{\prime}$ and $\rho^{\prime}$ for a given $Y^{\prime}$ are determined from the exact planar laminar flame solutions corresponding to the fuel temperatures $T_{0}=293 \mathrm{~K}$ (solid line) and $320 \mathrm{~K}$ (dashed line) and density $\rho_{0}$. Unlike $\tau_{\text {ind }}^{a}$, which is typically applicable in the context of an autoignition process, this quantity is often used to approximate the induction time in the flame. Nonetheless, for all $Y>0.15, \tau_{\text {reac }}$ provides a less accurate approximation of $\tau_{\text {ind }}$ than $\tau_{\text {ind }}^{a}$.

Finally, blue circles show the correlation time lags $\Delta t_{c}$ calculated in $\S 4$.1. There is close agreement between $\Delta t_{c}$ and both $\tau_{\text {ind }}$ and $\tau_{\text {ind }}^{a}$. Furthermore, when fuel heating is taken into account (dashed lines), all three quantities become 

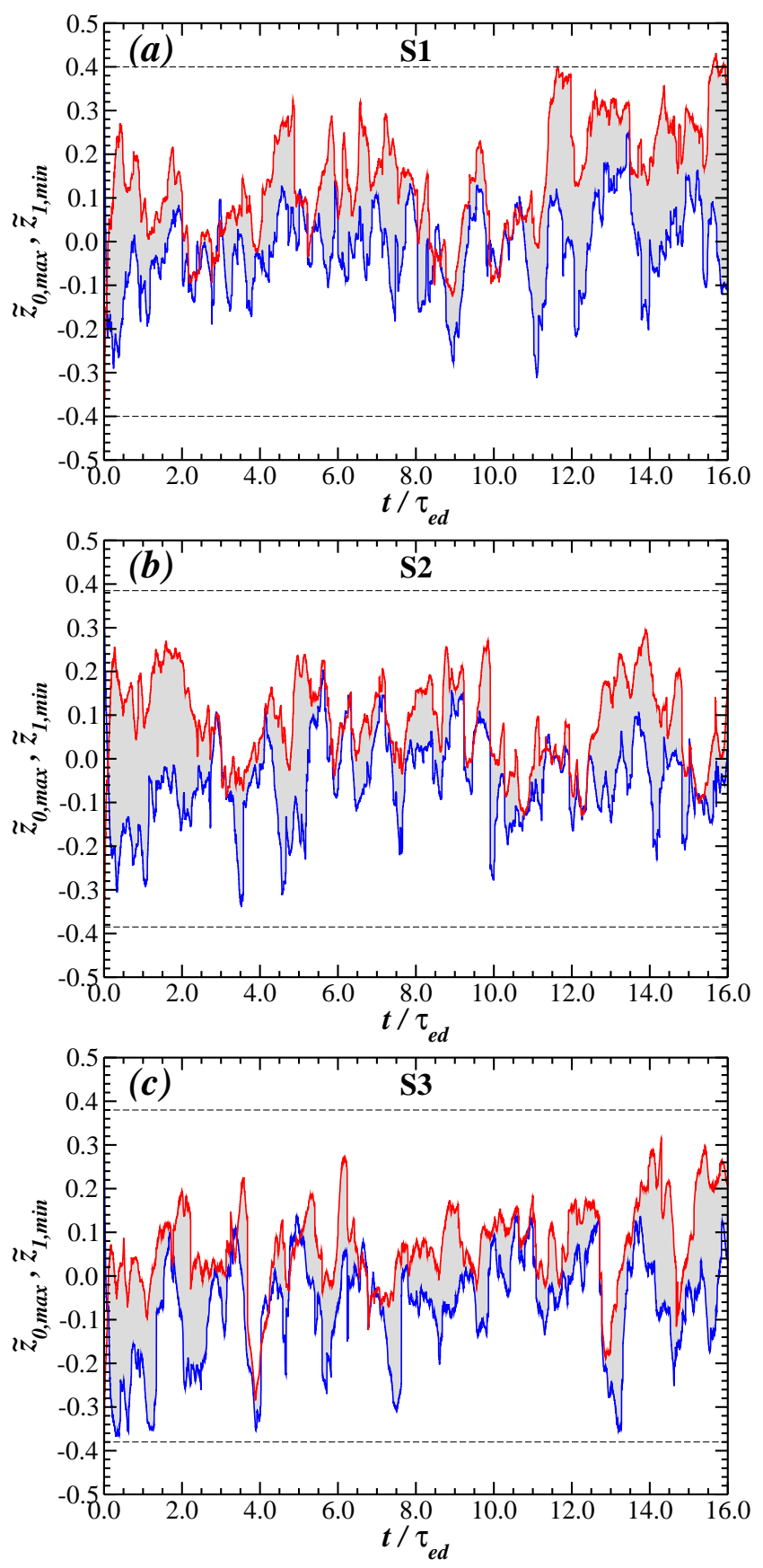

Figure B.19 Evolution of the normalized maximum extents of product and fuel penetration into the flame brush $\tilde{z}_{0, \max }$ and $\tilde{z}_{1, \min }$ (see Appendix A for the definition and Fig. 2 for the illustration) for simulations S1 (a), S2 (b), and S3 (c). The $\tilde{z}_{0, \max }$ is shown with red line, $\tilde{z}_{1, \min }$ - with blue line. Shaded regions mark the extent of macroscopic mixing of pure fuel and product inside the flame brush. Horizontal dashed lines show the average limiting values of $\tilde{z}_{0, \max }$ and $\tilde{z}_{1, \min }$ given by the condition (A.5) and calculated using the values of $\overline{\delta_{T} / \delta_{L}}$ listed in Table 4

even closer. 


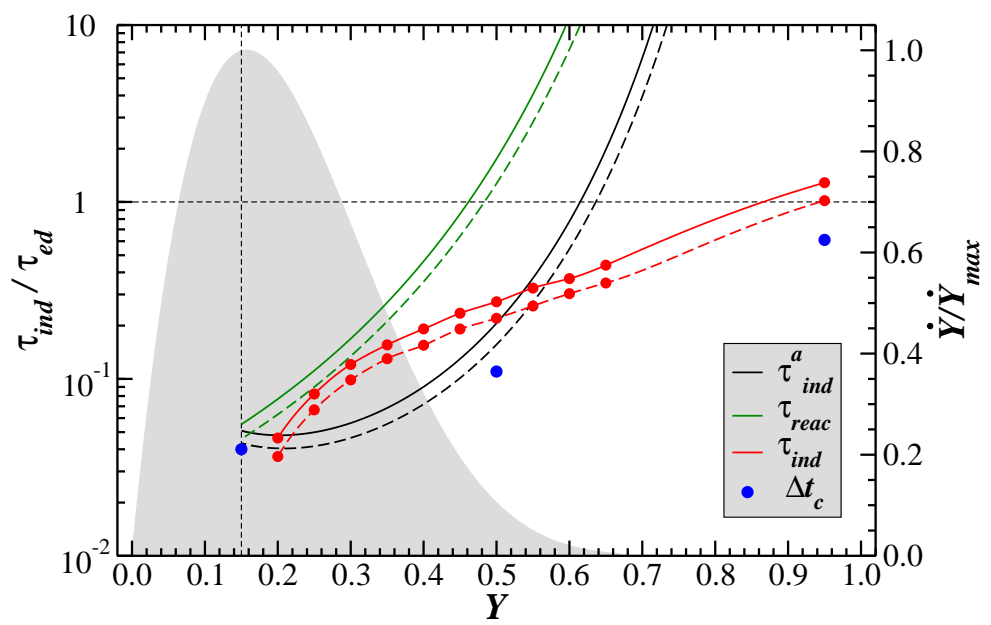

Figure B.20 Distributions of the actual induction times, $\tau_{\text {ind }}$, adiabatic induction times, $\tau_{\text {ind }}^{a}$, and the characteristic reaction times, $\tau_{\text {reac }}$, in the planar laminar flame. Also are shown the correlation time lags $\Delta t_{c}$ (cf. Fig. 6). Red circles show the calculated values of $\tau_{\text {ind }}$, red lines are the Akima spline fits. Green and black lines represent eqs. (20) and (B.1). Solid lines correspond to the fuel temperature $T_{0}=293 \mathrm{~K}$, dashed lines to $320 \mathrm{~K}$. Shaded gray region shows the distribution of the reaction rate, $\dot{Y}$, in the exact laminar flame solution normalized by its peak value $\dot{Y}_{\text {max }}=$ $9.5 \times 10^{4} \mathrm{~s}^{-1}$. The vertical dashed line indicates the region of peak reaction rate. The horizontal dashed line indicates equality between the induction time and the large-scale eddy turnover time, $\tau_{e d}$, during which turbulence completely reorganizes the turbulent flame structure. 


\section{References}

[1] G. Damköhler, Z. Elektrochemie und Angewandte Physikalische Chemie 46 (1940) 601-626. English translation: NASA TM No. 1112 (1947).

[2] R.W. Bilger, S.B. Pope, K.N.C. Bray, J.F. Driscoll, Proc. Combust. Inst. 30 (2005) 21-42.

[3] J.F. Driscoll, Prog. Energy Combust. Sci. 34 (2008) 91-134.

[4] A.N. Lipatnikov, J. Chomiak, Prog. Energy Combust. Sci. 28 (2002) 1-74.

[5] N. Peters, Turbulent Combustion, Cambridge University Press, 2000.

[6] Ya.B. Zel'dovich, Theory of Combustion and Detonation of Gases. Izd-vo AN SSSR, Moscow-Leningrad, 1944.

[7] C.K. Law, C.J. Sung, Prog. Energy Combust. Sci. 26 (2000) 459-505.

[8] E.S. Schetinkov, Physics of Gas Burning (Fizika Goreniya Gazov). Nauka, Moscow, 1965.

[9] A.J. Aspden, J.B. Bell, M.S. Day, S.E. Woosley, M. Zingale, Astrophys. J. 689 (2008) 1173-1185.

[10] A.J. Aspden, J.B. Bell, S.E. Woosley, Astrophys. J. 710 (2010) 1654-1663.

[11] A.Y. Poludnenko, E.S. Oran, Combust. Flame 157 (2010) 995-1011.

[12] A. Trouvé, T. Poinsot, J. Fluid Mech. 278 (1994) 1-31.

[13] D. Veynante, A. Trouvé, K.N.C. Bray, T. Mantel, J. Fluid Mech. 332 (1997) 263-293.

[14] M. Boger, D. Veynante, H. Boughanem, A. Trouvé, 27th Symp. (Int.) on Combustion, The Combustion Institute, Pittsburgh, (1998) 917-925.

[15] M. Tanahashi, Y. Nada, Y. Ito, T. Miyauchi, Proc. Combust. Inst. 29 (2002) 2041-2049.

[16] D. Thévenin, O. Gicquel, J.D. Charentenay, R. Hilbert, D. Veynante, Proc. Combust. Inst. 29 (2002) $2031-2039$.

[17] N. Chakraborty, R.S. Cant, Num. Heat Trans. A. Appl. 50 (2006) 623-643.

[18] J.A. van Oijen, R.J.M. Bastiaans, G.R.A. Groot, L.P.H. de Goey, Flow Turb. Combust. 75 (2005) 67-84

[19] S.H. Kim, H. Pitsch, Phys. Fluids 19 (2007) 115104-13.

[20] J.B. Bell, M.S. Day, J.F. Grcar, Proc. Combust. Inst. 29 (2002) 1987-1993.

[21] E.R. Hawkes, J.H. Chen, Combust. Flame 144 (2006) 112-125.

[22] A.N. Lipatnikov, J. Chomiak, Prog. Energy Combust. Sci. 36 (2010) 1-102.

[23] A.M. Khokhlov, Astrophys. J. 449 (1995) 695-713.

[24] P.A. Libby, F.A. Williams, in: P.A. Libby, F.A. Williams (Eds.), Turbulent Reacting Flows, Academic Press, $1994,1-61$.

[25] F.A. Williams, in: A. Yoshida (Ed.), Smart Control of Turbulent Combustion, Springer-Verlag, 2001, 1-11.

[26] V.N. Gamezo, T. Ogawa, E.S. Oran, Combust. Flame 155 (2008) 302-315.

[27] J.M. Stone, T.A. Gardiner, P. Teuben, J.F. Hawley, J.B. Simon, Astrophys. J. Supp. 178 (2008) 137-177.

[28] T.A. Gardiner, J.M. Stone, J. Comp. Phys. 227 (2008) 4123-4141.

[29] P. Colella, J. Comp. Phys. 87 (1990) 171-200.

[30] J. Saltzman, J. Comp. Phys. 115 (1994) 153-168.

[31] P. Colella, P.R. Woodward, J. Comp. Phys. 54 (1984) 174-201.

[32] A.Y. Poludnenko, T.A. Gardiner, E.S. Oran, Naval Research Lab. Memorandum Report (2010), in press.

[33] A. Kolmogorov, Dokl. Akad. Nauk SSSR 31 (1941) 538-541.

[34] J.M. Stone, E.C. Ostriker, C.F. Gammie, Astrophys. J. 508 (1998) L99-L102.

[35] M.N. Lemaster, J.M. Stone, Astrophys. J. 691 (2009) 1092-1108.

[36] Ya.B. Zeldovich, G.I. Barenblatt, V.B. Librovich, G.M. Makhviladze, The Mathematical Theory of Combustion and Explosions, Consultants Bureau, New York, 1985.

[37] L. Pan, J.C. Wheeler, J. Scalo, Astrophys. J. 681 (2008) 470-481.

[38] A.G. Istratov, V.B. Librovich, Astronautica Acta 14 (1969) 453-467.

[39] B. Karlovitz, D.W. Denniston, Jr., F.E. Wells, J. Chem. Phys. 19 (1951) 541-547.

[40] B. Lewis, G. von Elbe, Combustion, Flames, and Explosion of Gases, Academic Press, London, 1987.

[41] Ya.B. Zel'dovich, J. Appl. Mech. \& Tech. Phys. 7 (1966) 68-69.

[42] Ya.B. Zel'dovich, A.G. Istratov, N.I. Kidin, V.B. Librovich, Combustion Sci. Tech. 24 (1980) 1-13.

[43] L.D. Landau, Zh. Eksperim. i Teor. Fiz. 14 (1944) 6; L.D. Landau, E.M. Lifshitz, Fluid Mechanics, Pergamon Press, Oxford, 1987.

[44] J.M. Duclos, D. Veynante, T. Poinsot, Combust. Flame 95 (1993) 101-117. 